\title{
VERTICAL D4-D2-D0 BOUND STATES ON K3 FIBRATIONS AND MODULARITY
}

\author{
VINCENT BOUCHARD ${ }^{1}$, THOMAS CREUTZIG ${ }^{1}$, DUILIU-EMANUEL DIACONESCU ${ }^{2}$, CHARLES \\ DORAN $^{1,3}$, CALLUM QUIGLEY $^{1,4}$, ARTAN SHESHMANI $^{5,6}$
}

\begin{abstract}
An explicit formula is derived for the generating function of vertical D4-D2-D0 bound states on smooth K3 fibered Calabi-Yau threefolds, generalizing previous results of Gholampour and Sheshmani. It is also shown that this formula satisfies strong modularity properties, as predicted by string theory. This leads to a new construction of vector valued modular forms which exhibits some of the features of a generalized Hecke transform.
\end{abstract}

\section{Contents}

1. Introduction

1.1. Partition functions

1.2. The main formula

1.3. Modularity results Acknowledgments.

2. Vertical D4-D2-D0 bound states on K3 fibrations

2.1. The vertical charge lattice

2.2. BPS indices and partition functions

2.3. Mathematical approach via Donaldson-Thomas invariants

3. Lattice polarization and Noether-Lefschetz loci

3.1. Noether-Lefschetz numbers

3.2. Local systems and jump loci

3.3. Noether-Lefschetz numbers and modular forms

4. Vertical BPS indices from adiabatic IIA/heterotic duality

4.1. Primitive charge vectors

4.2. Singular fibers and adiabatic IIA/heterotic duality

4.3. Generating functions for primitive charge vectors

4.4. Non-primitive charge vectors and multicover contributions

5. Recursive derivation from stable pair invariants

5.1. Background

5.2. A recursive algorithm for $D T(r, d, n)$

5.3. Vertical Donaldson-Thomas invariants from Gopakumar-Vafa invariants 
5.4. Proof of the combinatorial identity 39

6. Modularity of partition functions $\quad 41$

6.1. The statement 41

6.2. A second proof of modularity 44

Appendix A. Weil representations and sublattices of the K3 lattice 499

Appendix B. Shintani's formula $\quad 50$

References $\quad 53$

\section{INTRODUCTION}

The main goal of this paper is to derive an explicit formula for all BPS degeneracies of vertical D4-D2-D0 bound states on K3 fibered Calabi-Yau threefolds $X$, subject to certain genericity conditions. Counting D4-D2-D0 BPS states is a natural problem in the context of IIA and M-theory compactifications, as well as IIA/heterotic duality. This problem is closely related to black hole entropy [14, 62, 63, 41, 53, 13, 16, as well as BPS algebras [28, 29]. Further ramifications include connections with quantum two dimensional Yang-Mills theory and topological strings [64, 2, 1, 31, as well as wallcrossing phenomena [50, 52, 51. As pointed out in [63, 48, 35] and studied in more depth in [17, a different connection with topological strings is provided by the Fourier-Mukai transform on elliptic fibrations. This problem is also firmly rooted in gauge theory, since it can be regarded as a string theoretic generalization of Vafa-Witten theory [65] on a K3 surface. Exact results for this case have been obtained in [48, 40]. At the same time, this problem has received a fair amount of attention in the mathematical literature [22, 23, 61, 24, 59, 60], in the framework of Dolandson-Thomas invariants of stable sheaves on Calabi-Yau threefolds

As shown in [65, 20, 15, 16], on physics grounds the generating function for D4-D2-D0 BPS indices is expected to have strong modularity properties. This has been confirmed by many explicit computations for stable torsion-free sheaves supported on rational surfaces [25, 68, 69, 170, 71, 26, 27, 44, 42, 45, 21, 59, 60]. These results can be placed in the context of Donaldson-Thomas theory if such sheaves are viewed as two dimensional torsion modules supported on rigid divisors in CalabiYau threefolds. For two dimensional sheaves supported on the fibers of a K3 pencil, modularity has been shown in certain cases in [22], as discussed in more detail below.

1.1. Partition functions. The geometric framework for this paper is that of lattice polarized K3 fibered projective Calabi-Yau threefolds satisfying certain natural genericity assumptions. In particular all fibers are assumed to be irreducible algebraic K3 surfaces with at most simple nodal singularities. In addition, one also assumes the existence of a lattice polarization defined as in [46, 36] in terms of an auxiliary smooth K3 fibration obtained by extremal transitions. Very briefly, a lattice polarization is defined by specifying a sublattice $\Lambda$ of the Picard group of $X$ satisfying certain conditions listed in Section 3 . This is a sublattice of the middle cohomology lattice $H^{2}(S, \mathbb{Z})$ of a 
smooth generic K3 surface such that the restriction of the intersection form to $\Lambda$ is a non-degenerate bilinear form of signature $(1, \operatorname{rk} \Lambda-1)$. Moreover, for the class of threefolds under consideration, $\Lambda$ will be naturally identified with the Picard lattice of any sufficiently generic smooth K3 fiber of $X$. As explained in Section 2. Poincaré duality then leads to a natural identification of the lattice of vertical curve classes on $X$ with the dual lattice $\Lambda^{\vee}$.

For further reference note that $\Lambda$ is naturally identified with a finite index sublattice of $\Lambda^{\vee}$ using the given bilinear pairing. Since $\Lambda^{\vee}$ is contained in $\Lambda_{\mathbb{Q}}=\Lambda \otimes_{\mathbb{Z}} \mathbb{Q}$, the same bilinear pairing induces a $\mathbb{Q}$-valued pairing on $\Lambda^{\vee}$ which will be denoted by $\left(d, d^{\prime}\right) \mapsto d \cdot d^{\prime} \in \mathbb{Q}$. Moreover, as the symmetric bilinear form is diagonalizable over $\mathbb{R}$, any element $d \in \Lambda$ admits a canonical decomposition $d=d_{+}+d_{-}$, with $d_{+}, d_{-} \in \Lambda_{\mathbb{R}}=\Lambda \otimes_{\mathbb{Z}} \mathbb{R}$, such that $\left(d_{+}\right)^{2} \geq 0,\left(d_{-}\right)^{2} \leq 0$ and $d_{+} \cdot d_{-}=0$.

In this context, the main object of study of this work is the thermal partition function constructed by Denef and Moore and [16] for D4-D2-D0 bound states, which is reviewed in Section 2, Alternative constructions in M-theory fivebranes have been carried out in [20, 15]. The approach of [16] will be adopted in this paper since it is based on D-brane effective actions, hence can be readily generalized to higher rank theories. The framework employed in [16] is an Euclidean IIA compactification on $X$ where the time direction is periodic with period $T$. Given the decomposition $H^{2}(X, \mathbb{Z}) \simeq \Lambda \oplus \mathbb{Z}\langle D\rangle$, the background $B$-field will be an element $-B \in \Lambda_{\mathbb{R}}$. Similarly, the background RR 3-form field will be given by $C_{3}=-C d t / T, C \in \Lambda_{\mathbb{R}}$, where $0 \leq t<T$ is the Euclidean time coordinate. The vertical components have been set to zero since they have trivial restriction to the K3 fibers, and the signs are included for later convenience. Let also $C_{1}=C_{0} d t / T$ be the expectation value of the RR 1-form, with $C_{0} \in \mathbb{R}$, and $\tau=C_{0}+i T / g_{s}$ where $g_{s}$ is the IIA string coupling.

The discrete topological invariants of vertical D4-D2-D0 configurations on $X$ are triples $\gamma=$ $(r, d, n)$ where $r, n \in \mathbb{Z}$ are $\mathrm{D} 4$ and anti-D0 multiplicities respectively, while $d \in \Lambda^{\vee}$ is the D2 charge. The BPS indices counting such states will be denoted by $\Omega(\gamma)$. By analogy with [16], it is shown in Section 2.2 that the thermal partition function can be written as a finite sum

$$
Z_{B P S}(X, r ; \tau, \bar{\tau}, B, C)=\sum_{\delta \in \Lambda^{\vee} / r \Lambda} Z_{B P S}(X, r, \delta ; \tau) \Theta_{r, \delta}^{*}(\tau, \bar{\tau} ; C, B)
$$

over equivalence classes $\delta \in \Lambda^{\vee} / r \Lambda$. For each such equivalence class,

$$
Z_{B P S}(X, r, \delta ; \tau)=\sum_{n} \Omega(\gamma) e^{2 \pi i \tau\left(n+d^{2} / 2 r-1\right)}
$$

and

$$
\Theta_{r, \delta}^{*}(\tau, \bar{\tau} ; C, B)=\sum_{\alpha \in \Lambda} e^{-2 \pi i \tau(d+r \alpha+r B / 2)_{-}^{2} / 2 r-2 \pi i \bar{\tau}(d+r \alpha+r B)_{+}^{2} / 2 r+2 \pi i(d+r \alpha+r B / 2) \cdot C} .
$$

where $d \in \Lambda^{\vee}$ is any representative of $\delta$. Note that the above sum is actually the complex conjugate of a Siegel Jacobi theta function of a coset of the rescaled lattice $\sqrt{r} \Lambda \subset \Lambda_{\mathbb{R}}$. As shown in Section 2 the above series are independent of the choice of $d$. 
As pointed out in [3], note that such an expression does not hold in general for arbitrary D4D2-D0 BPS states on Calabi-Yau threefolds. The fact that it holds here relies on the invariance of vertical BPS indices under endomorphisms of the charge lattice of the form (2.3), which is proven in Section 2.3. Moreover, a similar decomposition of the thermal partition function occurs in [16, Sect. 2.2] with one significant difference. In the cases studied in loc. cit. the generic supersymmetric D4-D2-D0 configuration consists of a single D4-brane wrapping a smooth very ample divisor in $X$ bound to some D2 and anti-D0 branes. In the present case, a generic stable configuration consists of a rank $r$ stable vector bundle supported on a smooth K3 fiber, and cannot be deformed to a single D4-brane wrapping a smooth surface. This is reflected in the presence of the cosets $\Lambda^{\vee} / r \Lambda$ with $r \geq 1$ in the right hand side of (2.10), as opposed to the analogous formulas in [16, Sect. 2.2]. This will turn out to have some important arithmetic consequences.

Mathematically the BPS indices $\Omega(\gamma)$ are generalized Donaldson-Thomas invariants [58, 38, 32] counting semistable vertical sheaves on $X$ suppported on K3 fibers. More precisely, the formalism of [38, 32] yields rational invariants $D T(\gamma) \in \mathbb{Q}$ which are conjecturally related to the integral ones $\Omega(\gamma)$ by the multicover formula

$$
D T(\gamma)=\sum_{\substack{k \in \mathbb{Z}, k \geq 1 \\ \gamma=k \gamma^{\prime}}} \frac{1}{k^{2}} \Omega\left(\gamma^{\prime}\right)
$$

Some aspects of generalized Donaldson-Thomas invariants are reviewed in Section 2.3.

The importance of rational invariants in this context was emphasized by Manschot in [43, where it is shown that partition functions of rational as opposed to integral invariants are expected to exhibit modular properties. This is indeed the case for the explicit formulas derived in this paper, as explained below.

The partition function of rational invariants is obtained by summing over multicovers. In order to write an explicit formula, note that for any $r, r^{\prime} \in \mathbb{Z}, r, r^{\prime} \geq 1$, such that $r=k r^{\prime}$ with $k \in \mathbb{Z}$ there is an injective morphism

$$
f_{r^{\prime}, k}: \Lambda^{\vee} / r^{\prime} \Lambda \rightarrow \Lambda^{\vee} / r \Lambda, \quad f_{r^{\prime}, k}\left([d]_{r^{\prime}}\right)=[k d]_{r} \text { for all } d \in \Lambda^{\vee}
$$

Here $[d]_{s} \in \Lambda^{\vee} / s \Lambda$ denotes the equivalence class of $d \in \Lambda^{\vee} \bmod s \Lambda$ for any $s \in \mathbb{Z}, s \geq 1$. Then the rank $r \geq 1$ partition function for rational invariants reads

$$
Z_{D T}(X, r ; \tau, \bar{\tau}, B, C)=\sum_{\delta \in \Lambda^{\vee} / r \Lambda} Z_{D T}(X, r, \delta ; \tau) \Theta_{r, \delta}^{*}(\tau, \bar{\tau} ; C, B)
$$

where

$$
Z_{D T}(X, r, \delta ; \tau)=\sum_{\substack{k \in \mathbb{Z}, k \geq 1 \\(r, \delta)=\left(k r^{\prime}, f_{r^{\prime}, k}\left(\delta^{\prime}\right)\right)}} \frac{1}{k^{2}} Z_{B P S}\left(X, r^{\prime}, \delta^{\prime} ; k \tau\right)
$$


1.2. The main formula. The main result of this paper is an explicit conjectural formula for the partition function (1.2). This formula will be written in terms of a certain vector valued modular form $\widetilde{\Phi}$ associated to an irreducible nodal $\mathrm{K} 3$ pencil $X \rightarrow C$ via the construction of [46, 36]. As explained in detail in Section 3, this form encodes the Noether-Lefschetz numbers of an associated smooth pencil $\widetilde{X}$ over a double cover of $C$. The form $\widetilde{\Phi}$ takes values in the Weil representation determined by the lattice $\Lambda$ equipped with the intersection form, and has weight $11-\operatorname{rk}(\Lambda) / 2$. Leaving the technical details for section 3.3. note that the latter is a representation of the metaplectic cover of $S L(2, \mathbb{Z})$ on the vector space $V=\mathbb{C}\left[\Lambda^{\vee} / \Lambda\right]$. The components of $\widetilde{\Phi}$ with respect to the natural basis of $\left(e_{g}\right), g \in \Lambda^{\vee} / \Lambda$ will be denoted by $\widetilde{\Phi}_{g}$. Note also that for any $l \geq 1$ there is a $\mathbb{Q} / \mathbb{Z}$-valued quadratic form $\theta_{l}$ on $\Lambda^{\vee} / l \Lambda$ by

$$
\theta_{l}\left([d]_{l}\right)=\frac{d^{2}}{2 l} \bmod \mathbb{Z}
$$

Then the main formula reads

$$
Z_{D T}(X, r, \delta ; \tau)=\frac{1}{2 r^{2}} \sum_{\substack{k, l \in \mathbb{Z}, k, l \geq 1 \\ k l=r, \delta=f_{l, k}(\eta)}} \sum_{s=0}^{l-1} l e^{-2 \pi i s \theta_{l}(\eta)}\left(\Delta^{-1} \widetilde{\Phi}_{[\eta]_{1}}\right)\left(\frac{k \tau+s}{l}\right)
$$

where $\Delta(\tau)=\eta(\tau)^{24}$. Note that in the right hand side $\eta \in \Lambda^{\vee} / l \Lambda$ is uniquely determined by $\delta$ since the morphism $f_{l, k}: \Lambda^{\vee} / l \Lambda \rightarrow \Lambda^{\vee} / r \Lambda$ is injective. Moreover $[\eta]_{1} \in \Lambda^{\vee} / \Lambda$ denotes the image of $\eta$ under the natural projection $\Lambda^{\vee} / l \Lambda \rightarrow \Lambda^{\vee} / \Lambda$.

Special cases of the above formula have been rigorously proven by Gholampour and Sheshmani 22. for K3 fibrations without singular fibers and primitive charge vectors as well as for rank $r=1$ invariants on irreducible nodal K3 pencils. Other special cases for arbitrary $r \geq 1$ were obtained in [17] for elliptic K3 pencils in Weierstrass form using the Fourier-Mukai transform.

A string theoretic derivation of equation (1.3) is given in Section 4 using adiabatic IIA/heterotic duality. The main idea is that for the purpose of vertical BPS index the K3 pencil can be regarded as a family of world sheet conformal field theories for a $T^{4}$ compactification of the $E_{8} \times E_{8}$ heterotic string. Such an identification is not canonical because the restriction of the Calabi-Yau threefold metric to a K3 surface is not in general hyper-Kähler. Nevertheless it can be used as a very efficient tool since the BPS index of D4-D2-D0 supported on a K3 fiber is invariant under metric perturbations. The conclusion is that counting D4-D2-D0 bound states supported on nodal fibers is equivalent to counting Dabholkar-Harvey states [14] in a smooth heterotic conformal field theory. Then one can use the general formulas for the degeneracies of such states obtained in [12.

At the same time, a mathematical derivation of the same formula is given in Section [5 using previous results of Gholampour, Sheshmani and Toda [24]. Using recursive methods, Section 5 gives a rigorous proof of equation (1.3) provided that one assumes the Gromov-Witten/stable pair correspondence [55] to hold. This proof is based on the explicit results obtained by Maulik and Pandharipande [46] for vertical Gopakumar-Vafa invariants of K3 pencils, as well as a remarkable 
combinatorial identity for the Fourier coefficients of a certain meromorphic Jacobi form of negative index.

1.3. Modularity results. The rank $r$ partition function is expected to be a non-holomorphic modular form of weight $(-3 / 2,1 / 2)$, as predicted in [20, 15, 16] on physics grounds. In order to show this for the explicit results derived in this paper, note that formula (1.3) yields the following expression

$$
\begin{aligned}
& Z_{D T}(X, r ; \tau, \bar{\tau}, B, C)= \\
& \frac{1}{2 r^{2}} \sum_{\substack{k, l \in \mathbb{Z}_{\geq 1} \\
k l=r}} \sum_{\eta \in \Lambda^{\vee} / l \Lambda} \sum_{s=0}^{l-1} l\left(\Delta^{-1} \widetilde{\Phi}_{[\eta]_{1}}\right)\left(\frac{k \tau+s}{l}\right) \Theta_{1,[\eta]_{1}}^{*}\left(\frac{k \tau+s}{l}, \frac{k \bar{\tau}+s}{l} ; k C+s B, l B\right) .
\end{aligned}
$$

This is proven in Section 4.4. Since $Z_{D T}(X, 1 ; \tau, \bar{\tau}, B, C)$ is a non-holomorphic modular function of weight $(-3 / 2,1 / 2)$ in $(\tau, \bar{\tau})$, the above formula is an order $r$ Hecke transform of the rank one result. Recall that the partition function of Vafa-Witten theory with gauge group $U(r)$ was previously shown in [48] to be given precisely by an order $r$ Hecke transform of its $U(1)$ counterpart. The above formula generalizes this construction to vertical D4-D2-D0 partition functions for K3 fibered Calabi-Yau threefolds.

As further discussed in Section 6, an important consequence of this result is that for fixed $r \geq 1$ the collection $\left(Z_{D T}(X, r, \delta ; \tau)\right), \delta \in \Lambda^{\vee} / r \Lambda$, forms a vector valued modular form for a certain representation of the metaplectic group $M p(2, \mathbb{Z})$ of weight $(-1-\operatorname{rk}(\Lambda) / 2)$. The representation in question is the dual Weil representation of the rescaled lattice $\sqrt{r} \Lambda \subset \Lambda_{\mathbb{R}}$. Since the above argument is rather indirect, a second direct proof of this statement is given in Section 6.2 for the skeptical reader.

It is also worth noting that these results yields a new transformation acting on vector valued modular forms which is somewhat similar to a construction carried out in [56, Thm. 3.1]. Given the present context, this construction could be regarded as a generalization of the Hecke transform to vector valued modular forms in the Weil representation. An interesting open question is whether this construction is related to the existing one in the mathematical literature [11]. These issues are currently under investigation in 8 .

Acknowledgments. We are very grateful to Terry Gannon for collaboration at the incipient stage of this project. D.E-D. owes special thanks to Steve Miller and Greg Moore for very helpful discussions and suggestions. In particular Section 2 grew out of illuminating discussions with Greg Moore during a string theory group meeting at Rutgers. A. S. would like to thank Yukinobu Toda for helpful discussions. We would also like to thank the referee for a careful reading of the manuscript and very insightful feedback. V.B and T. C. acknowledge the support of the Natural Sciences and Engineering Research Council of Canada. The work of D.-E. D. was partially supported by NSF grant DMS-1501612. C.F.D. would like to acknowledge support from the Natural 
Sciences and Engineering Research Council of Canada and the Visiting Campobassi Professorship of the University of Maryland. The work of C.Q. was supported in part through fellowships from the Pacific Institute for the Mathematical Sciences and the Natural Sciences and Engineering Research Council of Canada. A.S. would also like to thank Kavli IPMU for their kind and wonderful hospitality. His work at IPMU was supported by the World Premier International Research Center Initiative (WPI Initiative), MEXT, Japan.

\section{Vertical D4-D2-D0 Bound states on K3 Fibrations}

This section will review some basic facts on vertical supersymmetric D4-D2-D0 bound states on K3 fibered Calabi-Yau threefolds. The presentation will follow closely the treatment in Sections 2.1 and 2.2 of [16], adapting the construction of loc. cit. to K3 fibers rather than very ample divisors. Below $X$ will be a smooth projective Calabi-Yau threefold equipped with a projection map $\pi: X \rightarrow \mathbb{P}^{1}$ and a section $\sigma: \mathbb{P}^{1} \rightarrow X$. The Calabi-Yau condition implies that the generic fibers of $\pi$ will be smooth K3 surfaces. Singular fibers will be in general present, but throughout this paper it will be assumed that all such fibers have only isolated simple nodal points. It will be also assumed for simplicity that the integral cohomology of $X$ is torsion free and that $h^{1,0}(X)=0$. These assumptions are easily satisfied for sufficiently generic models.

2.1. The vertical charge lattice. For a type IIA compactification on $X$, the lattice of electric and magnetic charges of four dimensional BPS particles is isomorphic to the degree zero K-theory of $X$. In the absence of torsion, the lattice of charges can be identified with the even integral homology of $X$ using the Chern character and Poincaré duality. In particular the charges of D4-D2-D0 bound states supported on the fibers of $\pi$ take values in the sublattice

$$
\mathbb{Z}\langle D\rangle \oplus H_{2}(X, \mathbb{Z})^{\pi} \oplus H_{0}(X, \mathbb{Z}) \subset H_{\mathrm{even}}(X, \mathbb{Z}),
$$

where $D \in H_{4}(X, \mathbb{Z})$ is the $\mathrm{K} 3$ fiber class and $H_{2}(X, \mathbb{Z})^{\pi} \subset H_{2}(X, \mathbb{Z})$ is the kernel of the pushforward $\operatorname{map} \pi_{*}: H_{2}(X, \mathbb{Z}) \rightarrow H_{2}\left(\mathbb{P}^{1}, \mathbb{Z}\right)$.

Since one assumes the existence of a section, there are direct sum decompositions

$$
H_{4}(X, \mathbb{Z}) \simeq \mathbb{Z}\langle D\rangle \oplus \Lambda, \quad H_{2}(X, \mathbb{Z}) \simeq \mathbb{Z}\langle C\rangle \oplus H_{2}(X, \mathbb{Z})^{\pi}
$$

where $C, D$ are the section class and the $\mathrm{K} 3$ fiber class respectively. Moreover $\Lambda \subset H_{4}(X, \mathbb{Z})$ is the sublattice generated by horizontal 4-cycles relative to the map $\pi$, i.e. 4-cycles which project surjectively to $\mathbb{P}^{1}$. Poincaré duality yields a nondegenerate bilinear pairing

$$
H_{2}(X, \mathbb{Z})^{\pi} \times \Lambda \rightarrow \mathbb{Z}
$$

which identifies $H_{2}(X, \mathbb{Z})^{\pi}$ with the dual lattice $\Lambda^{\vee}$. In conclusion, the charges of vertical D4-D2-D0 bound states take values in a lattice of the form in

$$
\Gamma=\mathbb{Z} \oplus \Lambda^{\vee} \oplus \mathbb{Z} .
$$


A typical charge vector will be denoted by $\gamma=(r, d, n)$ where $r \geq 1$ is the D4 brane multiplicity, $d$ is the effective D2 charge and $n$ the anti-D0 brane charge, not including gravitational corrections.

Moreover, for any smooth fiber $X_{p}, p \in \mathbb{P}^{1}$, one has a natural restriction map

$$
H^{2}(X, \mathbb{Z}) \rightarrow H^{2}\left(X_{p}, \mathbb{Z}\right)
$$

whose image is contained in the sublattice of $(1,1)$ classes, $H^{1,1}\left(X_{p}\right) \cap H^{2}(X, \mathbb{Z})$. Using Poincaré duality, this yields a natural restriction map

$$
\Lambda \rightarrow H^{1,1}\left(X_{p}\right) \cap H^{2}(X, \mathbb{Z})
$$

which is furthermore injective for any smooth fiber $X_{p}$. For sufficiently generic models this map is in fact an isomorphism for any sufficiently generic smooth fiber $X_{p}$. However, it fails to be surjective at special points $p \in \mathbb{P}^{1}$, where the rank of $H^{1,1}\left(X_{p}\right) \cap H^{2}(X, \mathbb{Z})$ jumps. This jumping phenomenon is studied in detail in the mathematics literature on Noether-Lefschetz loci [46, 36]. The results used in this paper are reviewed in detail in Section 3 . For the purpose of this section, note that the map (2.1) will be assumed to be a primitive lattice embedding for all smooth fibers $X_{p}$. Moreover, note that the intersection pairing on $H^{2}\left(X_{p}, \mathbb{Z}\right)$ determines by restriction a nondegenerate symmetric bilinear form on $\Lambda$. Throughout this paper this form will be assumed to be independent of the point $p \in \mathbb{P}^{1}$. These conditions are part of the definition of a lattice polarized family of K3 surfaces, which is reviewed in detail in Section 3. In addition one also requires the signature of the induced form on $\Lambda$ to be $(1, \operatorname{rk}(\Lambda)-1)$.

In conclusion, $\Lambda$ will be endowed from this point on with a nondegenerate symmetric bilinear form which agrees with the intersection product on each smooth fiber. This form will be denoted by

$$
\left(\alpha, \alpha^{\prime}\right) \mapsto \alpha \cdot \alpha^{\prime}
$$

for any $\alpha, \alpha^{\prime} \in \Lambda$. By construction, this pairing is integral and even, but not unimodular. Therefore it determines an embedding $\Lambda \hookrightarrow \Lambda^{\vee}$ which will not be in general surjective. The quotient $\Lambda^{\vee} / \Lambda$ is a finite abelian group which will play a central part in this paper. Using this embedding $\Lambda$ will be identified in the following with a sublattice of $\Lambda^{\vee}$. Furthermore, the symmetric nondegenerate bilinear form on $\Lambda$ determines a $\mathbb{Q}$-valued symmetric nondegenerate bilinear form on $\Lambda_{\mathbb{Q}}=\Lambda \otimes_{\mathbb{Z}} \mathbb{Q}$, hence also on $\Lambda^{\vee}$, which is contained in $\Lambda_{\mathbb{Q}}$. This form will be similarly written as

$$
\left(d, d^{\prime}\right) \mapsto d \cdot d^{\prime}
$$

for any $d, d^{\prime} \in \Lambda^{\vee}$. Note that $d \cdot \alpha=d(\alpha) \in \mathbb{Z}$ for any $d \in \Lambda^{\vee}, \alpha \in \Lambda$.

The main goal of this paper is to derive explicit formulas for the supersymmetric indices of vertical D4-D2-D0 BPS configurations of fixed charge $\gamma$, for an arbitrary fixed Kähler class $\omega$ on $X$. 
2.2. BPS indices and partition functions. For each charge vector $\gamma$ there is a Hilbert space of four dimensional massive BPS particles $\mathcal{H}_{\omega}(X, \gamma)$ which carries an action of the three dimensional rotation group $S O(3) \subset S O(1,3)$. On general symmetry grounds this space is of the form

$$
\mathcal{H}_{\omega}(X, \gamma) \simeq 2((0)+(1 / 2)) \otimes \mathcal{H}_{\omega}^{\text {int }}(X, \gamma)
$$

where $\mathcal{H}_{\omega}^{\text {int }}(X, \gamma)$ is obtained by quantizing the internal degrees of freedom of these particles. The BPS index $\Omega_{\omega}(\gamma)$ is then defined as the Witten index

$$
\Omega_{\omega}(\gamma)=\operatorname{Tr}_{\mathcal{H}_{\omega}^{\text {int }}(X, \gamma)}(-1)^{2 J_{3}}
$$

where $J_{3} \subset S O(3)$ is the Cartan generator of the four dimensional little group.

Note that a simple application of the Kontsevich-Soibelman wallcrossing formula [38] shows that for vertical configurations the indices $\Omega_{\omega}(\gamma)$ are independent of the Kähler class $\omega$. This follows from the observation that $\left(\gamma, \gamma^{\prime}\right)=0$ for any two vertical charge vectors with respect to the natural symplectic pairing on $H^{\text {even }}(X, \mathbb{Z})$. Therefore the subscript $\omega$ will be omitted in the following.

One more important observation is that the BPS degeneracies $\Omega(r, d, n)$ are invariant under transformations of the form

$$
(r, d, n) \mapsto\left(r, d+r \alpha, n-d \cdot \alpha-\frac{1}{2} r \alpha^{2}\right),
$$

for any element $\alpha \in \Lambda$. This is proven at the end of Section 2.3 using the mathematical construction of BPS degeneracies as Donaldson-Thomas invariants.

As explained in Sections 2.1 and 2.2 of [16], there is a natural construction for generating functions of these indices derived from the natural DBI action on D4-branes. A central element in this construction is the jumping phenomenon associated to Noether-Lefschetz loci for K3 fibrations, which was briefly explained above. As opposed to the current case, the treatment of [16] is focused on a rank one D4-brane wrapping a smooth very ample divisor in $X$. However, their considerrations can be easily adapted to the situation at hand.

Suppose first the D4-brane rank is $r=1$. In this case a generic supersymmetric D4-D2-D0 configuration of charge $\gamma=(1, d, n)$ will be a bound state of one D4 brane and $k$ anti-D0 branes supported on an arbitrary smooth fiber $X_{p}$. One also has an $U(1)$ flux $\beta \in H^{2}\left(X_{p}, \mathbb{Z}\right)$ on the D4-brane such that

$$
\beta \cdot \alpha=d \cdot \alpha
$$

for any $\alpha \in \Lambda$. By supersymmetry, $F$ has to be a $(1,1)$ class. Note also that

$$
n=k-\frac{\beta^{2}}{2}
$$

by an easy application of the Grothendieck-Riemann-Roch theorem.

Recall that under the current assumptions $\Lambda$ is a sublattice of $H^{1,1}\left(X_{p}\right) \cap H^{2}\left(X_{p}, \mathbb{Z}\right)$, which is in turn a sublattice of $H^{2}\left(X_{p}, \mathbb{Z}\right)$. Let $\Lambda^{\perp} \subset H^{2}\left(X_{p}, \mathbb{Z}\right)$ denote the sublattice consisting of all elements 
which are orthogonal to $\Lambda$ with respect to the natural intersection pairing. Then note that there is a direct sum decomposition

$$
H^{2}\left(X_{p}, \mathbb{Q}\right) \simeq \Lambda_{\mathbb{Q}} \oplus \Lambda_{\mathbb{Q}}^{\perp}
$$

Let $\beta=\beta^{\|}+\beta^{\perp}$ be the corresponding decomposition of $\beta$. Using the natural inclusion $\Lambda^{\vee} \subset \Lambda_{\mathbb{Q}}$ equation (2.4) yields an identification $\beta^{\|}=d$.

The construction of the string theoretic generating function for the BPS degeneracies will follow closely Sections 2.1 and 2.2 of [16]. The generating function will be a thermal partition function working in an euclidean four dimensional background of the form $\mathbb{R}^{3} \times S^{1}$, where the time direction is periodic, with period $T$. The background B-field will be an element $-B \in \Lambda$ while the bacground RR fields will be set to

$$
C_{3}=-C d t / T, \quad C_{1}=C_{0} d t / T
$$

where $C \in \Lambda_{\mathbb{R}}, C_{0} \in \mathbb{R}$, and $0 \leq t<T$ is the Euclidean time coordinate. As in equation (2.9) of loc. cit. the contribution of all such supersymmetric configurations supported on a fixed smooth fiber $X_{p}$ to the partition function will be given by

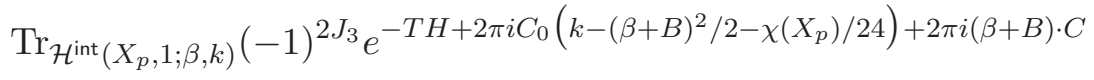

where $H$ is the Hamiltonian acting on the internal Hilbert space $\mathcal{H}^{\text {int }}\left(X_{p}, 1 ; \beta, k\right)$. For any cohomology class $\alpha \in H^{2}\left(X_{p}, \mathbb{R}\right)$ let $\alpha_{+}, \alpha_{-} \in H^{2}\left(X_{p}, \mathbb{R}\right)$ denote the self-dual, respectively anti-self-dual parts of $\alpha$ with respect to the restriction $\left.\omega\right|_{X_{p}}$ of the Kähler form. Then, evaluating this trace as in equations (2.10)-(2.14) of loc. cit. results in an expression of the form

$$
c\left(X_{p}, 1, \beta, k\right) e^{2 \pi i \tau\left(k-\left(\beta_{-}+B_{-}\right)^{2} / 2-\chi\left(X_{p}\right) / 24\right)-2 \pi i \bar{\tau}\left(\beta_{+}+B_{+}\right)^{2} / 2+2 \pi i(\beta+B) \cdot C}
$$

where

$$
\tau=C_{0}+\frac{i T}{g_{s}}, \quad c\left(X_{p}, 1, \beta, k\right)=\operatorname{Tr}_{\mathcal{H}^{\text {int }}\left(X_{p}, 1 ; \beta, k\right)}(-1)^{2 J_{3}} .
$$

Next recall that by assumption $\left.\omega\right|_{X_{p}} \in \Lambda_{\mathbb{R}}$, hence $\beta_{+}=\beta_{+}^{\|}$. This yields

$$
k-\beta_{-}^{2} / 2=n+\beta_{+}^{2} / 2=n+\left(\beta_{+}^{\|}\right)^{2} / 2=n+\left(\beta^{\|}\right)^{2} / 2-\left(\beta_{-}^{\|}\right)^{2} / 2,
$$

where $\beta^{\|}=d$. Moreover, since $B \in \Lambda_{\mathbb{R}}$, note that $B_{-}, B_{+} \in \Lambda_{\mathbb{R}}$, hence

$$
\beta_{-} \cdot B_{-}=\beta_{-}^{\|} \cdot B_{-} .
$$

Similarly,

$$
(\beta+B) \cdot C=\left(\beta^{\|}+B\right) \cdot C
$$

Since $\chi\left(X_{p}\right)=24$, one obtains

$$
e^{2 \pi i \tau\left(k-\left(\beta_{-}+B_{-}\right)^{2} / 2-\chi\left(X_{p}\right) / 24\right)-2 \pi i \bar{\tau}\left(\beta_{+}+B_{+}\right)^{2} / 2}=e^{2 \pi i \tau\left(n+d^{2} / 2-1\right)} e^{-2 \pi i \tau\left(d_{-}+B_{-}\right)^{2} / 2-2 \pi i \bar{\tau}\left(d_{+}+B_{+}\right)^{2} / 2}
$$


which depends only on $(d, n)$ and $B$. Then, summing over $(\beta, k)$, one obtains

$$
\begin{aligned}
& \sum_{\beta, k} c\left(X_{p}, 1, \beta, k\right) e^{2 \pi i \tau\left(k-(\beta+B)_{-}^{2} / 2-\chi\left(X_{p}\right) / 24\right)-2 \pi i \bar{\tau}(\beta+B)_{+}^{2} / 2+2 \pi i(\beta+B) \cdot C}= \\
& \sum_{d, n} \Omega\left(X_{p}, 1, d, n\right) e^{2 \pi i \tau\left(n+d^{2} / 2-1\right)} e^{-2 \pi i \tau(d+B)_{-}^{2} / 2-2 \pi i \bar{\tau}(d+B)_{+}^{2} / 2+2 \pi i(d+B) \cdot C},
\end{aligned}
$$

where

$$
\Omega\left(X_{p}, 1, d, n\right)=\sum_{\beta, \beta \|=d} c\left(X_{p}, 1, \beta, n+\beta^{2} / 2\right) .
$$

Moreover, note that $\Omega\left(X_{p}, 1, d, n\right)$ and $n+d^{2} / 2$ are invariant under transformations of the form (2.3) with $r=1$. Therefore for any $d \in \Lambda^{\vee}$, the sum

$$
\sum_{n} \Omega\left(X_{p}, 1, d, n\right) e^{2 \pi i \tau\left(n+d^{2} / 2-1\right)}
$$

depends only on the equivalence class $\delta=[d]_{1} \in \Lambda^{\vee} / \Lambda$ of $d \bmod \Lambda$. Hence it will be denoted by $Z\left(X_{p}, 1, \delta ; \tau\right)$. At the same time, for any fixed $d \in \Lambda^{\vee}, B, C \in \Lambda_{\mathbb{R}}$ note that

$$
\sum_{\alpha \in \Lambda} e^{-2 \pi i \tau(d+B+\alpha)_{-}^{2} / 2-2 \pi i \bar{\tau}(d+B+\alpha)_{+}^{2} / 2+2 \pi i(d+B+\alpha) \cdot C}=e^{-\pi i B \cdot C} \Theta_{\delta}^{*}(\tau, \bar{\tau} ; C, B)
$$

where $\Theta_{\delta}(\tau, \bar{\tau} ; C, B)$ is a Siegel Jacobi theta function, which also depends only on the equivalence class $\delta$ of $d \bmod \Lambda$. In conclusion for any smooth fiber $X_{p}$, the sum (2.7) can be written as

$$
\sum_{\delta \in \Lambda^{\vee} / \Lambda} Z\left(X_{p}, 1, \delta ; \tau\right) \Theta_{\delta}^{*}(\tau, \bar{\tau} ; C, B)
$$

up to the constant phase factor $e^{-\pi i B \cdot C}$, which can be omitted by a suitable choice of normalization of the partition function.

In order to compute the full degeneracies $\Omega(\gamma)$ one has to integrate over all possible locations of the fiber $X_{p}$, including singular fibers. The mathematical framework for such a computation is the theory developed by Behrend [5] which allows one to write $\Omega(\gamma)$ as the integral of a certain constructible function on the moduli space of supersymmetric D4-D2-D0 configurations. Leaving the details for Sections 2.3 and 4.1, it suffices to note here that the effect of integrating over all fibers is to convert $\Omega\left(X_{p}, 1, d, n\right)$ into $\Omega(1, d, n)$ leaving the general form of the partition function unchanged. In conclusion the partition function for rank $r=1$ invariants will take the form

$$
Z_{B P S}(X, 1 ; \tau, \bar{\tau}, B, C)=\sum_{\delta \in \Lambda^{\vee} / \Lambda} Z_{B P S}(X, 1, \delta ; \tau) \Theta_{\delta}^{*}(\tau, \bar{\tau} ; C, B)
$$

where

$$
Z_{B P S}(X, 1, \delta ; \tau)=\sum_{n} \Omega(1, d, n) e^{2 \pi i \tau\left(n+d^{2} / 2-1\right)}
$$

and $d \in \Lambda^{\vee}$ is an arbitrary fixed representative of $\delta$ for each $\delta \in \Lambda^{\vee} / \Lambda$. Note that $Z_{B P S}(X, 1, \delta ; \tau)$ is a power series in $q^{1 / 2 m}=e^{\pi i \tau / m}$ for any $\delta \in \Lambda^{\vee} / \Lambda$, where $m$ is the absolute value of the determinant of the intersection form on $\Lambda$. 
The next goal is to generalize the above construction to higher rank $r \geq 1$. This will be carried out in this section for primitive charge vectors $\gamma=(r, d, n)$. The general case can then be obtained by summation using the multicover formula for Donaldson-Thomas invariants, as shown in Section 4.3. For primitive charge vectors the generic supersymmetric D4-D2-D0 configuration is a D4-brane supported on a smooth fiber $X_{p}$ equipped with an $U(r)$ gauge field $A$. The topological invariants of $A$ are the first Chern class

$$
\beta=\operatorname{Tr}(F)
$$

and the second Chern class, or instanton number,

$$
k=\frac{\beta^{2}}{2}-\frac{\operatorname{Tr}\left(F^{2}\right)}{2}
$$

where $F$ is the field strength of $A$. Note again that

$$
n=k-\frac{\beta^{2}}{2} \quad \text { and } \quad \beta \cdot \alpha=d \cdot \alpha
$$

for any $\alpha \in \Lambda$. Moreover, $d=\beta^{\|}$with respect to the decomposition (2.6).

By supersymmetry the $U(r)$ gauge field must satisfy the Donaldson-Uhlenbeck-Yau equations. Therefore the field strength $F$ of $A$ must be a $(1,1)$ form on $X_{p}$ such that

$$
\omega_{p} \wedge F=\lambda \omega_{p}^{2}
$$

where $\omega_{p}=\left.\omega\right|_{X_{p}}$ is the restriction of the ambient Kähler class, and $\lambda=\left(\omega_{p} \cdot \beta\right) / \omega_{p}^{2}$ is constant. Now let $A_{0}=A-\operatorname{Tr}(A) / r$ be the traceless part of $A$. This is a $P U(r) \simeq S U(r) / \mu_{r}$ gauge field on $X_{p}$ with field strength

$$
F_{0}=F-\frac{\beta}{r} I_{r}
$$

Equation (2.9) implies that

$$
\omega_{p} \wedge F_{0}=0
$$

that is $A_{0}$ is an antiself-dual field configuration. Hence one has

$$
F_{-}=F_{0}+\beta_{-}, \quad F_{+}=\beta_{+} I_{r} .
$$

Let $k_{0}=-\operatorname{Tr}\left(F_{0}^{2}\right) / 2$, which is a rational number in $(1 / r) \mathbb{Z} \subset \mathbb{Q}$. Since $F_{0}$ is traceless, it follows that

$$
n=k_{0}-\frac{\beta^{2}}{2 r}
$$

The contribution of all such supersymmetric configurations to the thermal partition function will be given by

$$
\operatorname{Tr}_{\mathcal{H}^{\text {int }}\left(X_{p}, 1 ; \beta, k\right)}(-1)^{2 J_{3}} e^{-T H+2 \pi i C_{0}\left(k-(\beta+r B)^{2} / 2-\chi\left(X_{p}\right) / 24\right)+2 \pi i(\beta+r B) \cdot C}
$$

Evaluating this trace by analogy with equations (2.10)-(2.14) in [16] yields in this case

$$
c\left(X_{p}, r, \beta, k\right) e^{2 \pi i \tau\left(k_{0}-(\beta+r B)_{-}^{2} / 2 r-r \chi\left(X_{p}\right) / 24\right)-2 \pi i \bar{\tau}(\beta+r B)_{+}^{2} / 2 r+2 \pi i(\beta+r B) \cdot C} .
$$


By analogy with the rank one case, this expression is further equal to

$$
c\left(X_{p}, r, \beta, k\right) e^{2 \pi i \tau\left(n+d^{2} / 2 r-r\right)-2 \pi i \tau(d+r B)_{-}^{2} / 2 r-2 \pi i \bar{\tau}(d+r B)_{+}^{2} / 2 r+2 \pi i(d+r B) \cdot C} .
$$

Again, the BPS index $\Omega\left(X_{p}, \gamma\right)$ is obtained by summing over all pairs $(\beta, k)$ with $\beta^{\|}=d$ and $k=n+\beta^{2} / 2$ :

$$
\Omega\left(X_{p}, \gamma\right)=\sum_{\beta, \beta \|=d} c\left(X_{p}, r, \beta, n+\beta^{2} / 2\right) .
$$

Moreover, $\Omega\left(X_{p}, \gamma\right)$ and $n+d^{2} / 2 r$ are invariant under transformations of the form (2.3). Therefore for any $d \in \Lambda^{\vee}$, the sum

$$
\sum_{n} \Omega\left(X_{p}, \gamma\right) e^{2 \pi i \tau\left(n+d^{2} / 2 r-r\right)}
$$

depends only on the equivalence class $\delta=[d]_{r} \in \Lambda^{\vee} / r \Lambda$ of $d \bmod r \Lambda$. Hence it will be denoted by $Z\left(X_{p}, r, \delta ; \tau\right)$. At the same time, for any $d \in \Lambda^{\vee}$, the sum

$$
\sum_{\alpha \in \Lambda} e^{-2 \pi i \tau(d+r B+r \alpha)_{-}^{2} / 2 r-2 \pi i \bar{\tau}(d+r B+r \alpha)_{+}^{2} / 2 r+2 \pi i(d+r B+r \alpha) \cdot C}
$$

also depends only on the equivalence class of $d \bmod r \Lambda$. As shown in Section 6 , this sum is in fact equal to $e^{-\pi i r B \cdot C} \Theta_{r, \delta}^{*}(\tau, \bar{\tau} ; C, B)$, where the complex conjugate for a Siegel Jacobi theta function for a coset of the rescaled lattice $\sqrt{r} \Lambda \subset \Lambda_{\mathbb{R}}$. Choosing again a suitable normalization, one obtains a final expression for the rank $r$ partition function of the form

$$
Z_{B P S}(X, r ; \tau, \bar{\tau}, B, C)=\sum_{\delta \in \Lambda^{\vee} / r \Lambda} Z_{B P S}(X, r, \delta ; \tau) \Theta_{r, \delta}^{*}(\tau, \bar{\tau} ; C, B)
$$

where

$$
Z_{B P S}(X, r, \delta ; \tau)=\sum_{n} \Omega(\gamma) e^{2 \pi i \tau\left(n+d^{2} / 2 r-1\right)}
$$

and $d \in \Lambda^{\vee}$ is a fixed arbitrary representative for each equivalence class $\delta \in \Lambda^{\vee} / r \Lambda$. Note that $Z_{B P S}(X, r, \delta ; \tau)$ is a power series in $q^{1 / 2 m r}=e^{\pi i \tau / m r}$ for any $\delta \in \Lambda^{\vee} / \Lambda$, where $m$ is the absolute value of the determinant of the intersection form on $\Lambda$.

2.3. Mathematical approach via Donaldson-Thomas invariants. Mathematically, supersymmetric D4-D2-D0 bound states on $X$ are Bridgeland stable objects in the derived category of $X$. In this paper it will be assumed that for fixed numerical invariants Bridgeland stability reduces at large radius to Gieseker-stability for coherent sheaves. Therefore a supersymmetric D4-D2-D0 configuration will be a Gieseker semistable torsion coherent sheaf $E$ with respect to a certain polarization $\omega$ on $X$. For vertical D4-D2-D0 configurations $E$ will be also required to be set theoretically supported on a finite union of K3 fibers. Using the isomorphism $H_{2}(X, \mathbb{Z})^{\pi} \simeq \Lambda^{\vee}$ found in the previous section, such a sheaf has numerical invariants $\gamma=(r, d, n) \in \mathbb{Z}_{\geq 1} \times \Lambda^{\vee} \times \mathbb{Z}$ where

$$
\operatorname{ch}_{1}(E)=r D, \quad \operatorname{ch}_{2}(E)=d, \quad \operatorname{ch}_{3}(E)=-n \operatorname{ch}_{3}\left(\mathcal{O}_{x}\right) .
$$


For completeness recall Gieseker stability for pure dimension two sheaves on $X$. Given a real ample class $\omega$ on $X$, for any such nonzero sheaf $E$ let

$$
\mu_{\omega}(E)=\frac{\omega \cdot \operatorname{ch}_{2}(E)}{\omega^{2} \cdot \operatorname{ch}_{1}(E) / 2}, \quad \nu_{\omega}(E)=\frac{\chi(E)}{\omega^{2} \cdot \operatorname{ch}_{1}(E) / 2} .
$$

Then Gieseker (semi)stability with respect to $\omega$ is defined by the conditions

$$
\mu_{\omega}\left(E^{\prime}\right)(\leq) \mu_{\omega}(E)
$$

for any proper nonzero subsheaf $0 \subset E^{\prime} \subset E$, and

$$
\nu_{\omega}\left(E^{\prime}\right)(\leq) \nu_{\omega}(E)
$$

if the slope inequality (2.12) is saturated.

For any $\gamma=(r, d, n)$ let $M_{\omega}(\gamma)$ denote the coarse moduli space of $\omega$-semistable sheaves $E$ with discrete invariants (2.11). In the absence of semistable objects, the Donaldson-Thomas invariants $D T_{\omega}(\gamma)$ are defined in terms of virtual cycles; a special case of [58]. This is for example the case if $\gamma$ is primitive. Moreover in this case, there exists a constructible function $\nu: M_{\omega}(\gamma) \rightarrow \mathbb{Z}$ constructed by Behrend [5] such that

$$
D T_{\omega}(\gamma)=\chi\left(M_{\omega}(\gamma), \nu\right)
$$

By definition, given a constructible function $\phi: S \rightarrow \mathbb{Z}$ on any scheme of finite type, the weighted Euler characteristic $\chi(S, \phi)$ is defined by

$$
\chi(S, \phi)=\sum_{n \in \mathbb{Z}} n \chi\left(\phi^{-1}(n)\right),
$$

where $\chi()$ denotes the topological Euler characteristic.

The value of $\nu$ at a point $\mathfrak{m} \in M_{\omega}(\gamma)$ is determined by the local scheme structure of the moduli space near $\mathfrak{m}$. According to [9], the moduli space admits a local presentation as a critical locus of a polynomial function $W$ defined on the space of infinitesimal deformations of the corresponding sheaf $E$ on $X$. Then

$$
\nu(\mathfrak{m})=(-1)^{d}\left(1-\chi\left(M F_{\mathfrak{m}}(W)\right)\right.
$$

where $d$ is the dimension of the space of infinitesimal deformations and $M F_{\mathfrak{m}}(W)$ is the Milnor fiber of $W$ at $\mathfrak{m}$. Note that if the moduli space is smooth at $\mathfrak{m}$,

$$
\nu(\mathfrak{m})=(-1)^{\operatorname{dim}\left(\mathrm{T}_{\mathfrak{m}} \mathrm{M}_{\omega}(\gamma)\right)} .
$$

In particular, if the moduli space is a smooth projective variety, the constructible function $\nu$ takes the constant value $(-1)^{\operatorname{dim}\left(M_{\omega}(\gamma)\right)}$, hence

$$
\chi\left(M_{\omega}(\gamma), \nu\right)=(-1)^{\operatorname{dim}\left(\mathcal{M}_{\omega}(\gamma)\right)} \chi\left(M_{\omega}(\gamma)\right) .
$$

From a physical perspective, the infinitesimal deformations of $E$ are associated to complex chiral superfields in the low energy effective action of the corresponding D4-D2-D0 configuration, and $W$ 
is a superpotential interaction. The moduli space is locally identified with the critical locus of $W$. For an isolated critical point, Milnor's result [47] shows that $\nu(\mathfrak{m})$ is the same as the dimension of the chiral ring of $W$. Formula (2.15) provides a suitable generalization for non-isolated vacua.

In the presence of semistable objects, one has to employ the formalism of Kontsevich and Soibelman [38] or Joyce and Song [32] to construct generalized Donaldson-Thomas invariants $D T_{\omega}(\gamma) \in \mathbb{Q}$. For sufficiently generic $\omega$ there are conjectural integral invariants $\Omega_{\omega}(\gamma) \in \mathbb{Z}$ related to the rational ones by the multicover formula

$$
D T_{\omega}(\gamma)=\sum_{\substack{k \in \mathbb{Z}, k \geq 1 \\ \gamma=k \gamma^{\prime}}} \frac{1}{k^{2}} \Omega_{\omega}\left(\gamma^{\prime}\right)
$$

According to [18, 19] the integral invariants $\Omega_{\omega}(\gamma)$ are mathematical avatars of the BPS indices defined in (2.2). This justifies using the same notation in both cases. Moreover, as noted in the previous section, the wallcrossing formulas of [38, 32] show that these invariants are in fact independent of the Kähler class, therefore the subscript $\omega$ can be omitted.

Finally, to conclude this section, the following is a detailed proof of invariance of DonaldsonThomas invariants under the automorphisms (2.3) of the charge lattice.

First note that the transformations (2.3) are obtained by taking a tensor product by a line bundle $L$ on $X$. More precisely for any vertical sheaf $E$ one has the Chern class relations

$$
\begin{gathered}
\operatorname{ch}_{1}\left(E \otimes_{X} L\right)=\operatorname{ch}_{1}(E), \quad \operatorname{ch}_{2}\left(E \otimes_{X} L\right)=\operatorname{ch}_{2}(E)+c_{1}(L) \cdot \operatorname{ch}_{1}(E), \\
\operatorname{ch}_{3}\left(E \otimes_{X} L\right)=\operatorname{ch}_{3}(E)+c_{1}(L) \cdot \operatorname{ch}_{2}(E)+\frac{1}{2} c_{1}(L)^{2} \cdot \operatorname{ch}_{1}(E) .
\end{gathered}
$$

For $c_{1}(L)=\alpha$, the numerical invariants of $E$ change according to equation (2.3). Below it will be shown that taking a tensor product as above yields an isomorphism of moduli spaces for sufficiently generic Kähler classes. Since the vertical Donaldson-Thomas invariants do not change under wallcrossing, this implies the invariance statement needed in Section 2.

Suppose $F$ is a vertical pure dimension two sheaf with numerical invariants $\gamma=(r, d, n)$. Then $F \otimes_{X} L$ is also a vertical pure dimension two sheaf on $X$ with the same support as $F$ and numerical invariants as in (2.3) i.e.

$$
\tilde{\gamma}=\left(r, d+r \alpha, n-d \cdot \alpha-\frac{1}{2} r \alpha^{2}\right) .
$$

Let $\omega=t D+\eta$ be an arbitrary Kähler class on $X$, where $\eta \in \Lambda_{\mathbb{R}}$ is a relatively ample class. Since $D$ is orthogonal to all vertical curve classes with respect to the intersection product on $X$, and $D^{3}=0$ one can easily check that

$$
\mu_{\omega}(F)=\frac{2 d \cdot \eta}{r \eta^{2}}, \quad \nu_{\omega}(F)=\frac{4 r-2 n}{r \eta^{2}} .
$$

Note that $\chi(F)=2 r-n$ by Riemann-Roch. This yields

$$
\mu_{\omega}\left(F \otimes_{X} L\right)=\mu_{\omega}(F)+\frac{\alpha \cdot \eta}{\eta^{2}}, \quad \nu_{\omega}\left(F \otimes_{X} L\right)=\nu_{\omega}(F)+\frac{2 d \cdot \alpha}{r \eta^{2}}+\frac{\alpha^{2}}{\eta^{2}}
$$


Now let $E$ be a vertical sheaf as above and $0 \subset E^{\prime} \subset E$ be a nontrivial proper subsheaf. Then $E^{\prime}$ has to be vertical as well, hence it will have numerical invariants $\gamma^{\prime}=\left(r^{\prime}, d^{\prime}, n^{\prime}\right)$. Using the above formulas it follows that

$$
\mu_{\omega}\left(E \otimes_{X} L\right)-\mu_{\omega}\left(E^{\prime} \otimes_{X} L\right)=\mu_{\omega}(E)-\mu_{\omega}\left(E^{\prime}\right)
$$

and

$$
\nu_{\omega}\left(E \otimes_{X} L\right)-\nu_{\omega}\left(E^{\prime} \otimes_{X} L\right)=\nu_{\omega}(E)-\nu_{\omega}\left(E^{\prime}\right)+\frac{2}{\eta^{2}}\left(\frac{d}{r}-\frac{d^{\prime}}{r^{\prime}}\right) \cdot \alpha .
$$

Suppose $E$ is $\omega$-stable. This implies

$$
\mu_{\omega}(E)-\mu_{\omega}\left(E^{\prime}\right)>0
$$

or

$$
\mu_{\omega}(E)-\mu_{\omega}\left(E^{\prime}\right)=0 \quad \text { and } \quad \nu_{\omega}(E)-\nu_{\omega}\left(E^{\prime}\right)>0 .
$$

In the first case, equation (2.19) implies that

$$
\mu_{\omega}\left(E \otimes_{X} L\right)-\mu_{\omega}\left(E^{\prime} \otimes_{X} L\right)>0 .
$$

In the second case, note that

$$
\mu_{\omega}(E)-\mu_{\omega}\left(E^{\prime}\right)=\frac{2}{\eta^{2}}\left(\frac{d}{r}-\frac{d^{\prime}}{r^{\prime}}\right) \cdot \eta .
$$

For sufficiently generic $\eta \in \Lambda_{\mathbb{R}}$, equality of the slopes implies $d / r-d^{\prime} / r^{\prime}=0$. For example, if $\eta$ is a linear combination of lattice generators with sufficiently generic irrational coefficients. Then equation (2.20) further implies

$$
\nu_{\omega}\left(E \otimes_{X} L\right)-\nu_{\omega}\left(E^{\prime} \otimes_{X} L\right)=\nu_{\omega}(E)-\nu_{\omega}\left(E^{\prime}\right)>0 .
$$

To cover all cases, suppose $E$ is strictly $\omega$-semistable and let $0 \subset E^{\prime} \subset E$ be a proper nontrivial subsheaf. If $E^{\prime}$ does not saturate the stability condition, the proof is identical to the one given above. Hence suppose that

$$
\mu_{\omega}\left(E^{\prime}\right)=\mu_{\omega}(E), \quad \nu_{\omega}\left(E^{\prime}\right)=\nu_{\omega}(E)
$$

Then, under the same genericity assumption, the first equality implies again that $d / r=d^{\prime} / r^{\prime}$, which yields

$$
\mu_{\omega}\left(E^{\prime} \otimes_{X} L\right)=\mu_{\omega}\left(E \otimes_{X} L\right), \quad \nu_{\omega}\left(E^{\prime} \otimes_{X} L\right)=\nu_{\omega}\left(E \otimes_{X} L\right) .
$$

The map $E^{\prime} \mapsto E^{\prime} \otimes_{X} L$ is a bijection between the proper nontrivial subsheaves of $E$ and those of $E \otimes_{X} L$. Moreover, one can run the above argument in reverse taking a tensor product by $L^{-1}$. Therefore, for sufficiently generic $\eta \in \Lambda_{\mathbb{R}}$ it follows that $E$ is $\omega$-(semi)stable if and only if $E \otimes_{X} L$ is $\omega$-(semi) stable.

The plan for the rest of the paper is to provide two derivations for the expression (1.3) encoding all the above invariants. The first is a string theoretic derivation based on adiabatic IIA/heterotic duality while the second is based on the mathematical results of [24]. Both derivations rely heavily 
on a detailed understanding of lattice polarizations and Noether-Lefschetz loci, which is the subject of the next section.

\section{Lattice polarization and Noether-Lefschetz LOCI}

This section is a review of lattice polarized K3 fibrations and Noether-Lefshetz numbers mainly following [46, 36]. The presentation will be fairly technical by neccessity, since it lies the groundwork for the following sections.

Let $\pi: X \rightarrow \mathbb{P}^{1}$ be a K3 fibered smooth projective Calabi-Yau threefold with a section $\sigma: \mathbb{P}^{1} \rightarrow X$ satisfying the following conditions:

a) All K3 fibers are irreducible, reduced. The generic fiber is smooth and there are finitely many singular fibers, each of them with exactly one simple node. In order to simplify the presentation, it will also be assumed that the number of singular fibers is even, although this is not an essential assumption. All the following considerations extend with minor modifications to the fibrations with odd numbers of singular fibers.

Let $S^{\pi} \subset \mathbb{P}^{1}$ be the set of critical values of $\pi$. Under the above assumptions, $S^{\pi}$ is a finite set consisting of an even number of points. Let $f: \Sigma \rightarrow \mathbb{P}^{1}$ be a smooth generic double cover with branch locus $S^{\pi}$. Hence $\Sigma$ is a hyperelliptic curve of genus

$$
g(\Sigma)=\left|S^{\pi}\right| / 2-1 .
$$

Note that there is a unique ramification point of $f$ mapping to $\sigma \in S^{\pi}$. Abusing notation, it will be denoted by $\sigma$ as well, the distinction being clear from the context. The set of ramification points of $f$ will be denoted by $R^{f} \subset \Sigma$. Note also that if $\left|S^{\pi}\right|$ is odd one has to choose an extra point $\sigma_{\infty} \in \mathbb{P}^{1}$ parameterizing a generic smooth fiber, and consider a double cover with branch locus $S^{\pi} \cup\left\{\sigma_{\infty}\right\}$. All the following considerations will go through with minor modifications.

Let $X^{\prime}=X \times_{\mathbb{P}^{1}} \Sigma$. Then $X^{\prime}$ is a singular threefold with finitely many ordinary double points corresponding to the nodal points in the fibers of $\pi$. Under the current assumption there is one nodal point $x_{\sigma}^{\prime} \in X^{\prime}$ for each $\sigma \in S^{\pi}$. Let $\widetilde{X} \rightarrow X^{\prime}$ be a small crepant resolution of singularities. Let $\tilde{\pi}: \widetilde{X} \rightarrow \Sigma$ and $\tilde{p}: \widetilde{X} \rightarrow X$ be the natural projections. The exceptional locus consists of finitely many disjoint $(-1,-1)$ curves $\widetilde{C}_{\sigma}$ on $\widetilde{X}$, in one-to-one correspondence with points $\sigma \in S^{\pi}$. These are projective lines on $\widetilde{X}$ with normal bundles isomorphic to $\mathcal{O}(-1) \oplus \mathcal{O}(-1)$. Each such curve is at the same time a $(-2)$-curve on the fiber $\widetilde{X}_{\sigma}=\tilde{\pi}^{-1}(\sigma)$, which is an embedded resolution of the nodal surface $X_{\sigma}$.

Let $U$ denote the Lorentzian rank two lattice generated by two null vectors vectors $u, u^{*}$ with $u \cdot u^{*}=1$. Let $\Lambda_{K 3} \simeq U^{\oplus 3} \oplus \Lambda_{E_{8}}(-1)^{\oplus 2}$ be the middle homology lattice of a smooth generic K3 surface, where the $\Lambda_{E_{8}}(-1)$ denotes the $E_{8}$ root lattice equipped with a bilinear pairing given by the negative of the Cartan form. Let $\Lambda \subset \Lambda_{K 3}$ be a sublattice of rank $1 \leq \ell \leq 20$ and signature $(1, \ell-1)$, and let $\left(v_{1}, \ldots, v_{\ell}\right)$ be an integral basis of $\Lambda$. Let also $\Lambda^{\vee}$ be the dual lattice and $\left(\check{v}^{i}\right)$, $1 \leq i \leq \ell$ be the dual basis with respect to $\left(v_{i}\right)$. 
The pencil $\pi: X \rightarrow \mathbb{P}^{1}$ will be assumed to satisfy the following additional conditions, which are easily satisfied for generic complete intersections in toric varieties.

(b) There exists a collection of divisor classes $H_{1}, \ldots, H_{\ell} \in \operatorname{Pic}(X), m \geq 1$ such that the data $\left(\widetilde{X} \rightarrow \Sigma, \tilde{p}^{*} H_{1}, \ldots, \tilde{p}^{*} H_{\ell}\right)$ is a family of $\Lambda$-polarized K3 surfaces as defined in [36, Sect. 0.2.1]. In particular for any closed point $s \in \Sigma$ there is a primitive embedding $\Lambda \hookrightarrow \operatorname{Pic}\left(\widetilde{X}_{s}\right)$ mapping $v_{i}$ to $\widetilde{H}_{i, s}=\left.\tilde{p}^{*} H_{i}\right|_{\widetilde{X}_{s}}$ for all $1 \leq i \leq \ell$. One also requires the existence of an element $\lambda \in \Lambda$ which is mapped to a quasi-polarization of $\tilde{X}_{s}$ for each $s \in \Sigma$.

A stronger condition will be assumed here, namely that $\operatorname{Pic}(X) \simeq H^{2}(X, \mathbb{Z})$ is freely generated by $H_{1}, \ldots, H_{\ell}$ and the $\mathrm{K} 3$ fiber class $D$. Moreover, there is a relatively ample class on $X$ over $\mathbb{P}^{1}$ which restricts to $\lambda$ on each smooth fiber of $\pi$.

(c) For each point $\sigma \in S^{\pi}$ there is an orthogonal decomposition

$$
\operatorname{Pic}\left(\widetilde{X}_{\sigma}\right) \simeq \Lambda \oplus \mathbb{Z}\left\langle\widetilde{C}_{\sigma}\right\rangle
$$

with respect to the intersection product.

(d) For any sufficiently generic point $s \in \Sigma \backslash R^{f}$ the primitive embedding $\Lambda \hookrightarrow \operatorname{Pic}\left(\widetilde{X}_{s}\right)$ is an isomorphism.

3.1. Noether-Lefschetz numbers. Next recall the definition of Noether-Lefschetz numbers for the family $\tilde{\pi}: \widetilde{X} \rightarrow \Sigma$. Let $h \in \mathbb{Z}$ and $d=\sum_{i=1}^{\ell} d_{i} \check{v}^{i} \in \Lambda^{\vee}$. Since the bases $\left(v_{i}\right),\left(\check{v}^{i}\right)$ are fixed, $d$ will be often written as $d=\left(d_{i}\right)_{1 \leq i \leq \ell}$. Informally the Noether Lefschetz number $\widetilde{N L}_{h, d} \subset \Sigma$ is the number of points $s \in \Sigma$, counted with multiplicity, such that there exists a divisor class $\beta \in \operatorname{Pic}\left(\widetilde{X}_{s}\right)$ satisfying

$$
\beta^{2}=2 h-2, \quad \beta \cdot \widetilde{H}_{i, s}=d_{i}, \quad 1 \leq i \leq \ell
$$

A rigorous definition of Noether-Lefschetz numbers involves excess intersection theory, as shown in [46, Sect. 1.4] and [36, Sect. 0.2.2, 0.2.3]. Following [46, Sect. 1.4], consider the local system $\widetilde{\mathcal{V}}=R^{2} \tilde{\pi}_{*} \mathbb{Z}$ on $\Sigma$ and let $h: \widetilde{\mathcal{H}} \rightarrow \Sigma$ be the $\tilde{\pi}$-relative moduli space of Hodge structures of type $(1,20,1)$ on $\tilde{\mathcal{V}} \otimes_{\mathbb{Z}} \mathbb{C}$. For any pair $(h, d) \in \mathbb{Z} \times \mathbb{Z}^{\ell}$ there exists a countable union of divisors $\mathcal{D}_{h, d} \subset \widetilde{\mathcal{H}}$ parameterizing Hodge structures which contain a class $\beta \in \widetilde{\mathcal{V}}_{s}$ satisfying conditions (3.2). One also has a canonical section $\phi: \Sigma \rightarrow \widetilde{\mathcal{H}}$ such that $\phi(s)=\left[H^{0}\left(\widetilde{X}_{s}, \mathbb{C}\right)\right] \in \widetilde{\mathcal{H}}_{s}$ for any $s \in \Sigma$. Then

$$
\widetilde{N L}_{h, d}=\int_{\Sigma} \phi^{*} \mathcal{D}_{h, d}
$$

According to [46, Prop. 1] the right hand side of equation (3.3) is finite although $\mathcal{D}_{h, d}$ may have infinitely many components. The proof of [46, Prop. 1] shows that the image $\phi(\Sigma)$ intersects only finitely many of them. 
3.2. Local systems and jump loci. Given a pair $(h, d) \in \mathbb{Z} \times \Lambda^{\vee}$ and a point $s \in \Sigma$ let $\mathcal{B}_{s}^{\tilde{\pi}}(h, d)$ denote the set of classes $\beta \in \operatorname{Pic}\left(\widetilde{X}_{s}\right)$ satisfying conditions (3.2). This is a finite set by [46, Prop. 1]. The union $\mathcal{B}^{\tilde{\pi}}(h, d)=\cup_{s \in \Sigma} \mathcal{B}_{s}^{\tilde{\pi}}(h, d) \subset \widetilde{\mathcal{V}}$ decomposes as

$$
\mathcal{B}^{\tilde{\pi}}(h, d)=\widetilde{\mathcal{B}}^{\text {iso }}(h, d) \cup \widetilde{\mathcal{B}}^{\infty}(h, d)
$$

where $\widetilde{\mathcal{B}}^{\text {iso }}(h, d)$ projects to a finite subset of $\Sigma$, while $\widetilde{\mathcal{B}}^{\infty}(h, d) \subset \widetilde{\mathcal{V}}$ is a local system over $\Sigma$. For any pair $(h, d)$ let $J_{h, d}^{\tilde{\pi}} \subset \Sigma$ be the projection of $\widetilde{\mathcal{B}}^{\text {iso }}(h, d)$ to $\Sigma$. This finite subset of $\Sigma$ will be called the jump locus of type $(h, d)$. The Noether-Lefschetz numbers decompose accordingly as

$$
\widetilde{N L}_{h, d}=N L_{h, d}^{\text {iso }}+N L_{h, d}^{\infty} \text {. }
$$

The first term in the right hand side of (3.5) is a finite sum of the form

$$
N L_{h, d}^{\text {iso }}=\sum_{s \in J_{h, d}^{\tilde{\pi}}} \sum_{\beta \in \widetilde{\mathcal{B}}_{s}^{\text {iso }}(h, d)} \tilde{\mu}(h, d, \beta)
$$

where $\tilde{\mu}(h, d, \beta) \in \mathbb{Z}$ is the intersection multiplicity of the section $\phi(\Sigma)$ with $\mathcal{D}(h, d)$ at the closed point corresponding to $\beta$.

By definition, the second term in the right hand side of (3.5) is computed as follows. Note that there is a line bundle $\mathcal{K}=R^{0} \tilde{\pi}_{*} \omega_{\tilde{\pi}}$ on $\Sigma$, where $\omega_{\tilde{\pi}}$ is the relative dualizing sheaf. Then

$$
N L_{h, d}^{\infty}=-\int_{\widetilde{\mathcal{B}}^{\infty}(h, d)} c_{1}(\mathcal{K}) .
$$

Using the natural inclusion $\Lambda \hookrightarrow \Lambda^{\vee}$ determined by the intersection form, condition $(d)$ in this section implies that

$$
\widetilde{\mathcal{B}}_{s}^{\infty}(h, d)= \begin{cases}\{\alpha\} \subset \Lambda, & \text { if } d=\alpha \text { for some } \alpha \in \Lambda \text { and } h=1+\alpha^{2} / 2, \\ \emptyset, & \text { otherwise. }\end{cases}
$$

for any $s \in \Sigma$. Therefore $\mathcal{B}^{\infty}(h, d)$ is either empty or a rank one local system on $\Sigma$. Since $X$ is $K$-trivial, one then obtains

$$
N L_{h, d}^{\infty}= \begin{cases}-4, & \text { if } d=\alpha \text { for some } \alpha \in \Lambda \text { and } h=1+\alpha^{2} / 2, \\ 0, & \text { otherwise. }\end{cases}
$$

For future reference consider the following example. Let $\sigma \in \Sigma$ be a ramification point of $f$. Using the isomorphism (3.1), any class $\beta \in \operatorname{Pic}\left(\widetilde{X}_{\sigma}\right)$ is written as

$$
\beta=\alpha+l \widetilde{C}_{\sigma}
$$

with $l \in \mathbb{Z}$ and $\alpha \in \Lambda$. This implies that $\beta \cdot \widetilde{H}_{i, \sigma}=\alpha \cdot \widetilde{H}_{i, \sigma}$ for $1 \leq i \leq \ell$ and $\beta^{2}=\alpha^{2}-2 l^{2}$. Therefore, under the current assumptions, for any ramification point $\sigma \in R^{f}$, the component $\widetilde{\mathcal{B}}_{\sigma}^{\text {iso }}(h, d)$ is empty unless

$$
(h, d)=\left(1+\alpha^{2} / 2-l^{2}, \alpha\right)
$$


for some $\alpha \in \Lambda, l \in \mathbb{Z} \backslash\{0\}$, in which case

$$
\widetilde{\mathcal{B}}_{\sigma}^{\text {iso }}\left(1+\alpha^{2} / 2-l^{2}, \alpha\right) \simeq\left\{\alpha-l C_{\sigma}, \alpha+l C_{\sigma}\right\} .
$$

Furthermore, [36, Lemma 2], implies that

$$
\tilde{\mu}\left(1-l^{2}, 0, \pm l C_{\sigma}\right)=2
$$

for any $\sigma \in R^{f}, l \in \mathbb{Z} \backslash\{0\}$. More generally, by analogy with loc. cit., using condition (d) it can also be proved that

$$
\tilde{\mu}\left(1+\alpha^{2} / 2-l^{2}, \alpha \pm l C_{\sigma}\right)=2
$$

for any $\sigma \in R^{f}, \alpha \in \Lambda, l \in \mathbb{Z} \backslash\{0\}$.

One can similarly define local systems and jump loci for the restriction of the family $\pi: X \rightarrow \mathbb{P}^{1}$ to the open subset $U^{\pi}=\mathbb{P}^{1} \backslash S^{\pi}$. For any $p \in U^{\pi}$ and any $(h, d) \in \mathbb{Z} \times \Lambda^{\vee}$ let $\mathcal{B}_{p}^{\pi}(h, d)$ be the subset of classes $\beta \in \operatorname{Pic}\left(X_{p}\right)$ such that

$$
\beta^{2}=2 h-d,\left.\quad \beta \cdot H_{i}\right|_{X_{p}}=d_{i}, \quad 1 \leq i \leq \ell .
$$

By construction, $\mathcal{B}_{p}^{\pi}(h, d) \simeq \mathcal{B}_{s_{1}}^{\tilde{\pi}}(m, h, d) \simeq \mathcal{B}_{s_{2}}^{\tilde{\pi}}(m, h, d)$ for any $p \in U^{\pi}$ and any $(h, d)$, where $f^{-1}(p)=\left\{s_{1}, s_{2}\right\} \subset \Sigma$. In particular all $\mathcal{B}_{p}^{\pi}(h, d)$ are finite and the union $\mathcal{B}^{\pi}(h, d)=\cup_{p \in U^{\pi}} \mathcal{B}_{p}^{\pi}(h, d)$ decomposes again as

$$
\mathcal{B}^{\pi}(h, d)=\mathcal{B}^{\text {iso }}(h, d) \cup \mathcal{B}^{\infty}(h, d)
$$

by analogy with (3.4). Clearly, $\mathcal{B}_{p}^{\text {iso }}(h, d) \simeq \widetilde{\mathcal{B}}_{s_{1}}^{\text {iso }}(h, d) \simeq \widetilde{\mathcal{B}}_{s_{2}}^{\text {iso }}(h, d)$ and $\mathcal{B}_{p}^{\infty}(h, d) \simeq \widetilde{\mathcal{B}}_{s_{1}}^{\infty}(h, d) \simeq$ $\widetilde{\mathcal{B}}_{s_{2}}^{\infty}(h, d)$ for any $p \in U^{\pi}$. Again, the jump locus $J_{h, d}^{\pi} \subset U^{\pi}$ is the projection of $\mathcal{B}^{\text {iso }}(h, d)$. Obviously, $J_{h, d}^{\tilde{\pi}} \backslash R^{f}=f^{-1}\left(J_{h, d}^{\pi}\right)$. Moreover, one can again define the intersection multiplicity $\mu(h, d, \beta)$ for any $\beta \in \mathcal{B}^{\text {iso }}(h, d)$. This will coincide with the multiplicity of the corresponding classes $\beta_{i} \in \widetilde{\mathcal{B}}_{s_{i}}^{\text {iso }}(h, d)$, $1 \leq i \leq 2$. Then equation (3.11) yields

$$
N L_{h, d}^{\text {iso }}= \begin{cases}2 \sum_{p \in J_{h, d}^{\pi}} \sum_{\beta \in \mathcal{B}_{p}^{\text {iso }(h, d)}} \mu(h, d, \beta)+2\left|S^{\pi}\right|, & \text { if } d=\alpha, h=1+\alpha^{2} / 2-l^{2} \\ 2 \sum_{p \in J_{h, d}^{\pi}} \sum_{\beta \in \mathcal{B}_{p}^{\text {iso }(h, d)}} \mu(h, d, \beta) & \text { for some } \alpha \in \Lambda, l \in \mathbb{Z} \backslash\{0\}, \\ \text { otherwise, }\end{cases}
$$

which will be used in the computation of vertical D4-D2-D0 degeneracies in Section 4.2 .

3.3. Noether-Lefschetz numbers and modular forms. According to [7, 39] any smooth lattice polarized K3 pencil determines a vector valued modular form which encodes all its NoetherLefschetz numbers. This is briefly reviewed in [36, Sect. 0.2.4].

Let $m=|\operatorname{det}(M)|$, where $M_{i j}=v_{i} \cdot v_{j}$ is the intersection matrix of the basis of $\Lambda$. Let $G_{1}=\Lambda^{\vee} / \Lambda$, where the injection $\Lambda \hookrightarrow \Lambda^{\vee}$ is determined by the intersection form. As explained in Appendix $\mathrm{A}$ there is a canonical representation $\rho_{\Lambda}: M p(2, \mathbb{Z}) \rightarrow \operatorname{End}\left(\mathbb{C}\left[G_{1}\right]\right)$ constructed by Weil [67], where $M p(2, \mathbb{Z})$ is the metaplectic double cover of $S L(2, \mathbb{Z})$. 
Using the isomorphism (A.2), the main result of [7] yields the following modularity statement for Noether-Lefschetz numbers. For each pair $(h, d) \in \mathbb{Z} \times \Lambda^{\vee}, d=\left(d_{1}, \ldots, d_{\ell}\right)$, let

$$
\Delta(h, d)=(-1)^{\ell} \operatorname{det}\left(\begin{array}{cc}
M & d^{t} \\
d & 2 h-2
\end{array}\right) .
$$

Note that

$$
\frac{\Delta(h, d)}{2 m}=1+\frac{d^{2}}{2}-h
$$

Then there is a vector valued modular form

$$
\widetilde{\Phi}(q)=\sum_{\delta \in G_{1}} \widetilde{\Phi}_{\delta}(q) e_{\delta} \in \mathbb{C}\left[\left[q^{1 / 2 m}\right]\right] \otimes \mathbb{C}\left[G_{1}\right]
$$

of weight $w=(22-\ell) / 2$ and type $\rho_{\Lambda}$ such that

$$
\widetilde{N L}_{h, d}=\widetilde{\Phi}_{\delta}[\Delta(h, d) / 2 m] .
$$

where $[d]_{1}= \pm \delta$. Here $\Psi[s], s \in(1 / 2 m) \mathbb{Z} \subset \mathbb{Q}, s \geq 0$ are the Fourier coefficients of the series $\Psi(q) \in \mathbb{C}\left[\left[q^{1 / 2 m}\right]\right]$, that is

$$
\Psi(q)=\sum_{\substack{s \in(1 / 2 m) \mathbb{Z} \\ s \geq 0}} \Psi[s] q^{s} .
$$

As immediate consequence, this implies

$$
h \leq \frac{d^{2}}{2}+1
$$

for any class $\beta \in \mathcal{B}^{\tilde{\pi}}(h, d)$, which can be proved directly using the algebraic Hodge theorem.

Note that in its original form [7, Thm 4.5] implies the existence of such a vector valued modular form with values in $\rho_{\Lambda^{\perp}}^{*}$, where $\Lambda^{\perp} \subset \Lambda_{K 3}$ is the sublattice consisting of all elements $u \in \Lambda_{K 3}$, $u \cdot \Lambda=0$. The above statement follows from the isomorphism (A.2).

\section{Vertical BPS indices From adiabatic IIA/HEterotic DUALity}

This section consists of a string theoretic derivation of the main formula (1.3) from adiabatic IIA/heterotic duality for K3 fibrations.

4.1. Primitive charge vectors. Using the notation of Section 2.3. recall that the topological invariants of vertical two dimensional sheaves are given by triples $\gamma=(r, d, n) \in \mathbb{Z} \times \Lambda^{\vee} \times \mathbb{Z}$ where $\Lambda$ is the polarizing lattice of the K3 pencil $\pi: X \rightarrow \mathbb{P}^{1}$. The dual lattice $\Lambda^{\vee}$ is naturally identified with the sublattice $H_{2}(X, \mathbb{Z})^{\pi} \subset H_{2}(X, \mathbb{Z})$ parameterizing vertical curve classes. In this section, $\gamma=(r, d, n)$ will be assumed to be primitive. In this case all semistable vertical sheaves with invariants $\gamma$ are stable. According to [22, Lemma 3.1], any such sheaf $E$ must be the extension by zero of a stable torsion sheaf $F$ on a reduced fiber $X_{p}$ of $\pi$. For any $p \in \mathbb{P}^{1}$ let $M_{p}(\gamma)$ denote the closed subspace of the coarse moduli space parameterizing isomorphism classes of stable sheaves $E$ supported on $X_{p}$. Recall that $U^{\pi}=\mathbb{P}^{1} \backslash S^{\pi}$ is the open subset parameterizing smooth fibers. Let 
$M_{U^{\pi}}(\gamma)$ denote the open subset of the moduli space parameterizing stable sheaves $E$ supported on $X_{p}$ with $p \in U^{\pi}$. Then using equation (2.14) one has

$$
\Omega(\gamma)=\chi\left(M_{U^{\pi}}(\gamma), \nu\right)+\sum_{\sigma \in S^{\pi}} \chi\left(M_{\sigma}(\gamma), \nu\right)
$$

The first term in the right hand side of equation (4.1) can be explicitly evaluated using the results of [22]. To explain this in some detail, let $X_{p}$ be a smooth fiber of $\pi$ and $\iota_{p}: X_{p} \hookrightarrow X$ denote the natural embedding. Then any stable sheaf $E$ suported on $X_{p}$ is the extension by zero, $E \simeq \iota_{p *}(F)$, of an $\left.\omega\right|_{X_{p}}$-stable sheaf $F$ on $X_{p}$. The numerical invariants of $F$ are related to those of $E$ by the Grothendieck-Riemann-Roch formula:

$$
\operatorname{rk}(F)=r, \quad \iota_{*} \beta=d, \quad k-\frac{\beta^{2}}{2}=n,
$$

where $\beta=c_{1}(F)$ and $k=\int_{X_{p}} c_{2}(F)$. This implies that $M_{p}(\gamma)$ has disjoint components $M_{p, \beta}(\gamma)$ in one-to-one correspondence with classes $\beta \in \operatorname{Pic}(X)$ such that $\iota_{*} \beta=d$. Using the definition and the main properties of Noether-Lefschetz loci reviewed in Sections 3.1, respectively 3.2 and 3.3, the set of all such classes is a union

$$
\bigcup_{\substack{d \in \Lambda^{\vee}, h \in \mathbb{Z} \\ h \leq 1+d^{2} / 2}} \mathcal{B}_{p}^{\pi}(h, d) .
$$

where $h=1+\beta^{2} / 2$.

For each $\beta \in \mathcal{B}_{p}^{\pi}(h, d), k \in \mathbb{Z}$, let $M\left(X_{p}, r, \beta, k\right)$ be the moduli space of $\left.\omega\right|_{X_{p}}$-stable torsion free sheaves on $X_{p}$ with numerical invariants $(r, \beta, k)$, where $k=n+\beta^{2} / 2=n+h-1$. This is smooth and projective, of dimension

$$
\operatorname{dim} M\left(X_{p}, r, \beta, k\right)=2\left(r k-(r-1) \beta^{2} / 2-r^{2}+1\right)=2\left(r n-r^{2}+h\right) .
$$

In fact, according to [49] and [30, Sect. 6], for primitive invariants $(r, \beta, k)$, the moduli space $M\left(X_{p}, r, \beta, k\right)$ is a smooth deformation of a Hilbert scheme of points $H^{\operatorname{dim}\left(M\left(X_{p}, r, \beta, k\right)\right) / 2}(S)$ on a smooth algebraic K3 surface $S$.

One can easily construct a closed embedding $M\left(X_{p}, r, \beta, k\right) \hookrightarrow M_{p, \beta}(\gamma)$ which yields an isomorphism between the two sets of closed points. This implies that the reduced scheme $M_{p, \beta}(\gamma)^{\text {red }}$ is isomorphic to $M\left(X_{p}, r, \beta, k\right)$, hence $M_{p, \beta}(\gamma)$ has the same dimension as $M\left(X_{p}, r, \beta, k\right)$. In particular $M_{p, \beta}$ is nonempty if and only if

$$
h \geq r(r-n) .
$$

Therefore the disjoint components of $M_{p}(\gamma)$ are in one-to-one correspondence with elements of

$$
\bigcup_{\substack{d \in \Lambda^{\vee}, h \in \mathbb{Z} \\ r(r-n) \leq h \leq 1+d^{2} / 2}} \mathcal{B}_{p}^{\pi}(h, d),
$$

which is a finite set for fixed $\gamma=(r, d, n)$, possibly empty. Note however that in general $M_{p, \beta}(\gamma)$ will not be isomorphic to $M\left(X_{p}, r, \beta, k\right)$ as a scheme since its structure sheaf can in principle contain 
nilpotent elements. The different scheme structure of $M_{p, \beta}(\gamma)$ will lead to nontrivial values of the Behrend function, as explained below equation (4.10).

To summarize, one has a decomposition

$$
\chi\left(M_{p}(\gamma), \nu\right)=\sum_{\substack{h \in \mathbb{Z} \\ r(r-n) \leq h \leq 1+d^{2} / 2}} \sum_{\beta \in \mathcal{B}_{p}^{\pi}(h, d)} \chi\left(M_{p, \beta}(\gamma), \nu\right),
$$

where the sum in the right hand side is finite. Next recall that for any $p \in U^{\pi}=\mathbb{P}^{1} \backslash S^{\pi}$ the set $\mathcal{B}_{p}^{\pi}(h, d)$ decomposes as $\mathcal{B}_{p}^{\pi}(h, d)=\mathcal{B}_{p}^{\infty}(h, d) \cup \mathcal{B}_{p}^{\text {iso }}(h, d)$. Note that $M_{p, \beta}(\gamma)$ is an isolated closed component of the moduli space for each $\beta \in \mathcal{B}_{p}^{\text {iso }}(h, d)$. Using the results of [5], its contribution to the right hand side of (4.5) follows from [22, Thm. 3.18],

$$
\left.\chi\left(M_{p, \beta}(\gamma), \nu\right)=\mu(h, d, \beta) \chi\left(M\left(X_{p}, r, \beta, k\right)\right)=\mu(h, d, \beta) c(r(n-r)+h)\right),
$$

where

$$
c(r(n-r)+h)= \begin{cases}\chi\left(H^{r(n-r)+h}(S)\right), & \text { if } h \geq r(r-n), \\ 0 & \text { otherwise. }\end{cases}
$$

In the above formula $H^{k}(S)$ denotes the Hilbert scheme of $k$ points on a smooth generic algebraic K3 surface $S$. The coefficients $c(r(n-r)+h)$ are determined by Göettsche's formula [25] applied to K3 surfaces,

$$
q^{-1} \sum_{k=0}^{\infty} \chi\left(H^{k}(S)\right) q^{k}=\frac{1}{\eta(q)^{24}}
$$

The coefficient $\mu(h, d, \beta)$ is the same as the contribution of the isolated class $\beta$ to the NoetherLefschetz number in (3.6).

Furthermore, according to condition (e) in Section 3.2.

$$
\mathcal{B}_{p}^{\infty}(h, d)= \begin{cases}\mathcal{B}_{p}^{\infty}(\alpha)=\{\alpha\} \subset \Lambda, & \text { if }(h, d)=(h(\alpha), \alpha) \text { for some } \alpha \in \Lambda, \\ \emptyset, & \text { otherwise }\end{cases}
$$

where $h(\alpha)=1+\alpha^{2} / 2$. Therefore, if $d \neq \alpha$ for some $\alpha \in \Lambda$, the moduli space $M_{U^{\pi}}(\gamma)$ will be a finite union of isolated closed components whose contributions are given by (4.6). If $d=\alpha$ for some $\alpha \in \Lambda$, according to [22, Lemma 3.7], there is a smooth connected component $M_{U^{\pi}}^{\infty}(\gamma)$ of $M_{U^{\pi}}(\gamma)$ whose set of closed points coincides with the union $\cup_{p \in U^{\pi}} M_{p, \alpha}(\gamma)$. Moreover for each $p \in U$, there is an isomorphism $M_{p, \alpha}(\gamma) \simeq M\left(X_{p}, r, \alpha, k\right)$, with $k=n+\alpha^{2} / 2$. Since $M\left(X_{p}, r, \alpha, k\right)$ is smooth and projective of dimension (4.3), this implies that

$$
\chi\left(M_{p, \alpha}(\gamma), \nu\right)=-\chi\left(M_{p, \alpha}(\gamma)\right)=-c(r(n-r)+h(\alpha))
$$

and

$$
\chi\left(M_{U}^{\infty}(r, \alpha, n), \nu\right)=-\chi\left(U^{\pi}\right) c(r(n-r)+h(\alpha)) .
$$


Therefore, for any $\gamma=(r, d, n)$, the contribution of $M_{U^{\pi}}(\gamma)$ to the Donaldson-Thomas invariant $\Omega(\gamma)$ is

$$
\begin{aligned}
\chi\left(M_{U^{\pi}}(\gamma), \nu\right)=-\chi\left(U^{\pi}\right) c\left(r(n-r)+d^{2} / 2+1\right) \delta_{d, \Lambda} \\
+\sum_{\substack{h \in \mathbb{Z} \\
r(r-n) \leq h \leq d^{2} / 2+1}} \sum_{\beta \in \mathcal{B}^{\text {iso }}(h, \alpha)} \mu(h, d, \beta) c(r(n-r)+h),
\end{aligned}
$$

where

$$
\delta_{d, \Lambda}= \begin{cases}1, & \text { if } d \in \Lambda, \\ 0, & \text { otherwise }\end{cases}
$$

For completeness note that the weights $\mu(h, d, \beta)$ in equation (4.6) have a clear physical interpretation. This was first observed in a similar context in [16, App. G]. Namely, one can easily check that any vertical stable D4-D2-D0 configuration has exactly one normal infinitesimal deformation corresponding to translations along the base of the K3 fibration. More precisely, given a stable vertical sheaf $E=\iota_{p *}(F)$ supported on a reduced K3 fiber $X_{p}$ one can easily check that the space $\operatorname{Ext}_{X}^{1}(E, E)$ of infinitesimal deformations splits as

$$
\operatorname{Ext}_{X}^{1}(E, E) \simeq \operatorname{Ext}_{X_{p}}^{1}(F, F) \oplus \operatorname{Ext}_{X_{p}}^{0}(F, F) .
$$

The first summand parameterizes infinitesimal deformations of $F$ as a sheaf on $X_{p}$, while the second parameterizes normal deformations in the Calabi-Yau threefold $X$. Moreover, stability implies that Ext $_{X_{p}}^{0}(F, F) \simeq \mathbb{C}$ is one dimensional. This means that the low energy effective action of the corresponding D4-D2-D0 configuration will contain a complex chiral fields $\mathcal{X}_{1}, \ldots, \mathcal{X}_{d-1}$ associated to tangent fluctuations and an additional chiral field $\Phi$ associated to normal fluctuations to the fiber. Here $d=\operatorname{dim}_{\operatorname{Ext}_{X}^{1}}(E, E) \geq 1$. Since the moduli space $M\left(X_{p}, r, \beta, k\right)$ is smooth, the tangent deformations parameterized by $\mathcal{X}_{1}, \ldots, \mathcal{X}_{d-1}$ are unobstructed. However, if $\beta=c_{1}(F)$ is an isolated curve class on $X_{p}$, the normal deformations of $E$ will be obstructed. This will be encoded in a superpotential interaction $W\left(\mathcal{X}_{1}, \ldots, \mathcal{X}_{d}, \Phi\right)$ such that the critical scheme defined by $d W=0$ is locally isomorphic to a nilpotent extension of the moduli space $M\left(X_{p}, r, \beta, k\right)$. Then using equation (2.15), the value of the Behrend function $\nu([E])$ at the point $[E]$ will be determined by the resulting nilpotent extension. In principle, $\nu([E])$ may jump as $[E]$ moves in the moduli space. However, it is natural to conjecture it is constant along $M_{p, \beta}(\gamma)$ and takes value $\nu([E])=\mu(h, d, \beta)$ at all points. This is certainly in agreement with equation (4.6). While a rigorous proof would be quite difficult, intuitively one expects this to be the case since the only obstructions to the normal deformations of $E$ come from obstructions to normal deformations of the curve class $\beta$, which are independent of $E$.

4.2. Singular fibers and adiabatic IIA/heterotic duality. In order to finish the computation one has to evaluate the contributions of the singular fibers $X_{\sigma}, \sigma \in \Sigma$ to the right hand side of equation (4.1). The presence of singularities makes a direct geometric approach difficult. However one can gain important insight using fiberwise heterotic/IIA duality for the K3 fibration $\pi: X \rightarrow \mathbb{P}^{1}$. 
Since the Donaldson-Thomas invariants are independent of the Kähler class $\omega$, the latter can be chosen such that the volume of the section of $\pi$ is much larger than that of the K3 fibers. In this regime, it is natural to define a constructible function $\mu: \mathbb{P}^{1} \rightarrow \mathbb{Z}$,

$$
\mu(p)=\chi\left(M_{p}(\gamma), \nu\right)
$$

Clearly, the value of $\mu$ at $p$ represents the contribution of the fiber $X_{p}$ to the Donaldson-Thomas invariant. More concretely, one can write

$$
\Omega(\gamma)=\chi\left(\mathbb{P}^{1}, \mu\right)
$$

The main idea emerging from heterotic/IIA duality is that the value of $\mu$ at a point $p \in \mathbb{P}^{1}$ must be related to degeneracies of perturbative BPS states for a $T^{4}$ compactification of the $E_{8} \times E_{8}$ heterotic string. A concrete conjecture will be formulated below.

First recall that six dimensional heterotic/IIA duality identifies a $T^{4}$ compactification of the $E_{8} \times E_{8}$ heterotic string to a K3 compactification of the IIA string. The heterotic Narain lattice $\Gamma_{4,20}$ is isomorphic to a direct sum $U \oplus \Lambda_{K 3}$, where $\Lambda_{K 3} \simeq U^{\oplus 3} \oplus \Lambda_{E_{8}}(-1)^{\oplus 2}$ is the middle homology lattice of a smooth generic K3 surface. This identification singles out a topologically nontrivial circle $S_{A}^{1} \subset T^{4}$ corresponding to the first $U$ summand.

The conformal field theory moduli space $\mathcal{M}_{\text {het }}$ of the $E_{8} \times E_{8}$ heterotic string on $T^{4}$ is a quotient of the form

$$
\mathcal{M}_{\text {het }}=\operatorname{Aut}\left(\Gamma_{4,20}\right) \backslash \widetilde{\mathcal{M}}_{\text {het }}
$$

where $\widetilde{\mathcal{M}}_{\text {het }}=O^{+}(4,20) / S O(4) \times O(20)$ and $\operatorname{Aut}\left(\Gamma_{4,20}\right)$ is the automorphism group of the Narain lattice, acting naturally on the coset space. The latter is isomorphic to the grassmannian of spacelike 4-planes $\Pi \subset \Gamma_{4,20} \otimes_{\mathbb{Z}} \mathbb{R}$, hence it is a smooth complex manifold. The quotient by the $T$-duality group will have orbifold singularities.

According to [4, Thm. 6], a certain open subspace of this moduli space is precisely identified with the moduli space of conformally invariant nonlinear sigma models with target space K3, including metric and $B$-field moduli. Hence $\mathcal{M}_{\text {het }}$ is in fact a compactifiction of the sigma model moduli space. As explained for example in [4, Sect. 4.3] certain points in $\mathcal{M}_{\text {het }}$ correspond to nonperturbative IIA compactification on K3 surfaces with ADE quotient singularities. Such points are associated to six dimensional gauge symmetry enhancement. More precisely, for a generic point in the moduli space, the six dimensional gauge group is $U(1)^{24}$. Let $\widetilde{\mathcal{D}} \subset \widetilde{\mathcal{M}}_{\text {het }}$ be the locus where the spacelike 4-plane $\Pi$ is orthogonal to some vector $\beta \in \Gamma_{4,20}, \beta^{2}=-2$. Then the abelian gauge group is enhanced to $S U(2) \times U(1)^{23}$ at generic points on $\mathcal{D}=O\left(\Gamma_{4,20}\right) \backslash \widetilde{\mathcal{D}} \subset \mathcal{M}_{\text {het }}$. For the purposes of the present discussion, it should be emphasized that the points on $\mathcal{D}$ parameterize smooth well behaved heterotic conformal field theories, although the corresponding K3 surfaces in IIA theory develop $A_{1}$ singularities.

The duality also leads to a precise indentification of the Hilbert spaces of six dimensional BPS states in the two string theories, as explained for example in [29, 13]. As shown in [12, [13, 
Sect. 6.2], D4-D2-D0 BPS states with charge vector $\gamma=(r, \beta, k)$ supported on $S$ are in one-to-one correspondence with certain perturbative heterotic string states with momentum $k$ and winding number $r$ on the circle $S_{A}^{1}$. These states are obtained by tensoring the ground state of the right moving superconformal sector with a level $N$ state of the bosonic left moving sector, where $N$ is determined by level matching:

$$
N=r(k-r)-(r-1) \beta^{2} / 2+1 .
$$

These are the Dabholkar and Harvey states considered in [14] in relation to black hole entropy. A general formula for the degeneracies of such states is derived in [12, Sect 3].

Assuming $S$ to be algebraic, note that $N$ is half the dimension of the moduli space of stable torsion free sheaves on $S$. Since the left moving sector consists of 24 bosons, it follows that the degeneracy of these states is the $N$-th coefficient $c_{N}$ in the expansion of $q / \eta(q)^{24}$, in agreement with Goettsche's formula (4.7). This follows from [12, Sect. 3] as well as [13, Sect. 6].

Finally, suppose $S$ is a singular algebraic K3 surface with a single node corresponding to a generic point in $\widetilde{\mathcal{D}}$, and let $\widetilde{S}$ be its minimal crepant resolution. Let $\beta_{C} \subset H^{2}(\widetilde{S}, \mathbb{Z})$ denote the Poincaré dual of the exceptional (-2)-curve $C \subset \widetilde{S}$. Note that $\beta_{C}$ is identified with a root vector of one of the $E_{8}$ sublattices of $H_{2}(\widetilde{S}, \mathbb{Z})$. As explained above, the dual heterotic conformal field theory associated to $S$ is still smooth, except that the six dimensional gauge group of the corresponding six dimensional vacuum is enhanced to $S U(2)$. The extra massless $W$-bosons correspond to heterotic vertex operators associated to the root $\beta_{C}$. In particular the six dimensional theory exhibits a gauge symmetry which maps $\beta_{C} \mapsto-\beta_{C}$. This is the action of the generator of the Weyl group of the enhanced $S U(2)$ gauge group.

The degeneracies of DH states in the conformal theory associated to the nodal surface $S$ are exactly the same as those computed in the conformal field theory associated to the blow-up $\widetilde{S}$. This is manifest from the counting algorithm, which is independent of deformations of conformal field theory as long as the theory remains smooth. However, since the reflection $\beta_{C} \mapsto-\beta_{C}$ is a gauge symmetry, any two states DH related by this reflection are physically identical, so such a pair should be counted only once in the six dimensional BPS spectrum.

Returning to the family of $\Lambda$-polarized algebraic K3 surfaces $\pi: X \rightarrow \mathbb{P}^{1}$, note that this family cannot be canonically identified with a family of heterotic conformal field theories since the restriction of the Calabi-Yau threefold metric to a K3 fiber need not be hyper-Kähler. However, the BPS index for D4-D2-D0 states supported on a fiber is independent of metric perturbations, hence one can still derive a precise conjecture for the constructible function $\mu: \mathbb{P}^{1} \rightarrow \mathbb{Z}$ by counting perturbative heterotic string states. 
Using equations (4.5), (4.6) and (4.8), the contribution of the fiber $X_{p}, p \in U^{\pi}$, to the four dimensional BPS index of charge $\gamma=(r, d, n)$ is given by

$$
\mu(p)=-c\left(r(n-r)+d^{2} / 2+1\right) \delta_{d, \Lambda}+\sum_{\substack{h \in \mathbb{Z}, r(r-n) \leq h \leq d^{2} / 2+1}} \sum_{\beta \in \mathcal{B}^{\text {iso }}(h, d)} \mu(h, d, \beta) c(r(n-r)+h)
$$

The sum in the right hand side represents the contribution of all charge vectors $\beta \in H^{2}\left(X_{p}, \mathbb{Z}\right)$ which yield the same charge vector $\alpha$ with respect to the four-dimensional abelian gauge group i.e. $\iota_{*} \beta=\alpha$. Aside from the weights $\mu(h, d, \beta)$, the contribution of each class $\beta$ is given by the corresponding degeneracy of heterotic DH states. As explained below (4.10), for any class $\beta \in \mathcal{B}_{p}^{\text {iso }}(h, d)$, the weight $\mu(h, d, \beta)$ represents the vacuum multiplicity of the corresponding BPS D4-D2-D0 configurations supported on $X_{p}$.

Moreover, recall that the components $M_{p, \alpha}(\gamma)$ of the moduli space of D4-D2-D0 branes on $X_{p}$ fit in the smooth family $M_{U^{\pi}}^{\infty}(\gamma)$ over $U^{\pi}$. Therefore, according to formula (2.16), the contribution of degeneracies of states with $\beta=\alpha$ to the four dimensional index should be weighted by $(-1)$, which encodes their four dimensional spin quantum number.

Employing heterotic/IIA duality as above, it follows that the contribution $\mu(\sigma), \sigma \in S^{\pi}$, of a singular fiber can be inferred from counting D4-D2-D0 bound states supported on its blow-up $\widetilde{X}_{\sigma}$. The main point is that, choosing appropriate hyper-Kähler metrics on $X_{\sigma}, \widetilde{X}_{\sigma}$, one obtains smooth heterotic conformal field theories related by a smooth deformation. Hence the DH degeneracies as well as the four dimensional spin quantum number will be the same in the two theories. The only difference is the gauge symmetry $\beta_{C} \mapsto-\beta_{C}$ in the six dimensional vacuum associated to the nodal surface, which implies that DH states with charge $\alpha+l \beta_{C}$ are physically indistinguishable from $\mathrm{DH}$ states with charge $\alpha-l \beta_{C}$. Working under the genericity assumptions formulated in Section 3, the multiplicity of all curve classes supported on $\widetilde{X}_{\sigma}$ is given by equation (3.11). Therefore, collecting the facts, one is led to the following conjectural expression

$$
\mu(\sigma)=-c\left(r(n-r)+d^{2} / 2+1\right) \delta_{d, \Lambda}+\sum_{\substack{l \in \mathbb{Z}, l \geq 1 \\ l^{2} \leq r(n-r)+d^{2} / 2+1}} c\left(r(n-r)+d^{2} / 2+1-l^{2}\right) \delta_{d, \Lambda}
$$

for the contribution of a singular nodal fiber to the BPS index. Using equations (4.14) and (4.15) in equation (4.12), one then obtains

$$
\begin{aligned}
\Omega(\gamma)= & -\chi\left(\mathbb{P}^{1}\right) c\left(r(n-r)+d^{2} / 2+1\right) \delta_{d, \Lambda}+\sum_{\substack{h \in \mathbb{Z} \\
r(r-n) \leq h<d^{2} / 2+1}} \sum_{\beta \in \mathcal{B} \text { iso }(h, d)} \mu(h, d, \beta) c(r(n-r)+h) \\
& +\left|S^{\pi}\right| \sum_{\substack{l \in \mathbb{Z}, l \geq 1 \\
l^{2} \leq r(n-r)+d^{2} / 2+1}} c\left(r(n-r)+d^{2} / 2+1-l^{2}\right) \delta_{d, \Lambda} .
\end{aligned}
$$


Finally, using equations (3.9), (3.13) the above formula can be rewritten as

$$
\Omega(\gamma)=\frac{1}{2} \sum_{\substack{h \in \mathbb{Z} \\ r(r-n) \leq h \leq d^{2} / 2+1}} c(r(n-r)+h) \widetilde{N L}_{h, d}
$$

where $\widetilde{N L}_{h, d}$ are the Noether-Lefschetz numbers of the threefold $\widetilde{X}$ constructed in Section 3 ,

4.3. Generating functions for primitive charge vectors. Suppose the pair $(r, \delta) \in \mathbb{Z}_{\geq 1} \times$ $\Lambda^{\vee} / r \Lambda$ is primitive. This means there is no integer $k \in \mathbb{Z}, k \geq 1$, such that $k \mid r$ and $\delta=\left[k d^{\prime}\right]_{r}$ with $d^{\prime} \in \Lambda^{\vee}$. Then any charge vector $\gamma=(r, d, n)$ with $\delta=[d]_{r}$ will be primitive. Recall that the rank $r$ partition function of vertical D4-D2-D0 invariants is an expression of the form (2.10), where

$$
Z_{B P S}(X, r, \delta ; \tau)=\sum_{n \in \mathbb{Z}} \Omega(r, d, n) q^{n+d^{2} / 2 r-r} .
$$

In the above formula $d \in \Lambda^{\vee}$ is a fixed arbitrary representative of $\delta \in \Lambda^{\vee} / r \Lambda$. For any $r \geq 1$ let $G_{r}=\Lambda^{\vee} / r \Lambda$ and let $[d]_{r} \in G_{r}$ denote the equivalence class of $d \in \Lambda^{\vee}$. Let $h(d) \in \mathbb{Z}$ be defined by

$$
\frac{d^{2}}{2}+1=\frac{h(d)}{2 m}
$$

where $m=|\operatorname{det}(M)|$. Recall that the Noether-Lefschetz numbers $\widetilde{N L}_{h, d}$ are identified in equation (3.14) with the Fourier coefficients of a vector valued modular form $\widetilde{\Phi}(q)$ with values in the Weil representation of $\Lambda$. Then equations (3.14) and (4.16) yield

$$
Z_{B P S}(X, r, \delta ; \tau)=\frac{1}{2} \sum_{\substack{n \in \mathbb{Z} \\ r(r-n) \leq h(d) / 2 m}} q^{n+d^{2} / 2 r-r} \sum_{r(r-n) \leq h \leq h(d) / 2 m} c(r(n-r)+h) \widetilde{\Phi}_{[d]_{1}}[h(d) / 2 m-h],
$$

Let

with $k, l \in \mathbb{Z}$. In particular

$$
\frac{l}{2 m}=r(n-r)+\frac{h(d)}{2 m}, \quad k=r(n-r)+h
$$

$$
n-r=\frac{l-h(d)}{2 r m} \in \mathbb{Z}
$$

Then the right hand side of the above equation can be written as

$$
\begin{aligned}
& \frac{1}{2} q^{d^{2} / 2 r} \sum_{\substack{l \in \mathbb{Z}, l \geq 0,(l-h(d)) / 2 r m \in \mathbb{Z}}} q^{(l-h(d)) / 2 r m} \sum_{\substack{k \in \mathbb{Z} \\
0 \leq k \leq l / 2 m}} c(k) \widetilde{\Phi}_{[d]_{1}}[l / 2 m-k]= \\
& \frac{1}{4 r m} q^{d^{2} / 2 r} \sum_{l \in \mathbb{Z}, l \geq 0} q^{(l-h(d)) / 2 r m} \sum_{s=0}^{2 r m-1} e^{2 \pi i(l-h(d)) s / 2 r m} \sum_{\substack{k \in \mathbb{Z} \\
0 \leq k \leq l / 2 m}} c(k) \widetilde{\Phi}_{[d]_{1}}[l / 2 m-k] .
\end{aligned}
$$

Let $\Delta(q)=\eta(q)^{24}$, which is a modular form of weight $(-12)$. Note that $\Delta^{-1}(q)=q^{-1} \sum_{k \geq 0} c(k) q^{k}$ by Goettsche's formula (4.7). Then one has a series identity

$$
\sum_{l \in \mathbb{Z}, l \geq 0} q^{l / 2 r m} e^{2 \pi i l s / 2 r m} \sum_{\substack{k \in \mathbb{Z} \\ 0 \leq k \leq l / 2 m}} c(k) \widetilde{\Phi}_{[d]_{1}}[l / 2 m-k]=q^{1 / r} e^{2 \pi i s / r}\left(\Delta^{-1} \widetilde{\Phi}_{[d]_{1}}\right)\left(\frac{\tau+s}{r}\right) .
$$


Therefore

$$
\begin{aligned}
Z_{B P S}(X, r, \delta ; \tau) & =\frac{1}{4 r m} q^{d^{2} / 2 r} q^{(1-h(d) / 2 m) / r} \sum_{s=0}^{2 r m-1} e^{2 \pi i s(1-h(d) / 2 m) / r}\left(\Delta^{-1} \widetilde{\Phi}_{[d]_{1}}\right)\left(\frac{\tau+s}{r}\right) \\
& =\frac{1}{4 r m} \sum_{s=0}^{2 r m-1} e^{-\pi i s d^{2} / r}\left(\Delta^{-1} \widetilde{\Phi}_{[d]_{1}}\right)\left(\frac{\tau+s}{r}\right) .
\end{aligned}
$$

Since $\Delta^{-1} \widetilde{\Phi}(q)$ is a vector valued modular form of weight $(-1-\ell / 2)$ with values in the Weil representation $\rho_{\Lambda}$, one has

$$
\left(\Delta^{-1} \widetilde{\Phi}_{[d]_{1}}\right)\left(\frac{\tau+s+k r}{r}\right)=e^{\pi i k d^{2}}\left(\Delta^{-1} \widetilde{\Phi}_{[d]_{1}}\right)\left(\frac{\tau+s}{r}\right) .
$$

At the same time

$$
e^{-\pi i(s+k r) d^{2} / r}=e^{-\pi i k d^{2}} e^{-\pi i s d^{2} / r}
$$

Therefore each term in the right hand side of equation (4.17) is invariant under $s \mapsto s+k r, k \in \mathbb{Z}$. Then equation (4.17) reduces to

$$
Z_{B P S}(X, r, \delta ; \tau)=\frac{1}{2 r} \sum_{s=0}^{r-1} e^{-\pi i s d^{2} / r}\left(\Delta^{-1} \widetilde{\Phi}_{[d]_{1}}\right)\left(\frac{\tau+s}{r}\right) .
$$

In particular, for $r=1$ one obtains $Z_{B P S}(X, 1, \delta ; \tau)=\left(\Delta^{-1} \widetilde{\Phi}_{\delta}\right)(\tau)$ for any $\delta \in \Lambda^{\vee} / \Lambda$, in agreement with the results of [22]. As required by physical arguments [20, 15, 16], the collection $\left(Z_{B P S}(X, 1, \delta ; \tau)\right)_{\delta \in \Lambda^{\vee} / \Lambda}$ determines a weight $(-1-\ell / 2)$ vector-valued modular form with values in the Weil representation.

4.4. Non-primitive charge vectors and multicover contributions. To conclude this section suppose $\gamma$ is not primitive. Then in general there will exist strictly semistable objects in the moduli space of stable sheaves, making the theory of Donaldson-Thomas invariants more difficult. In particular one has the rational invariants $D T(\gamma)$ which are related to the integral ones $\Omega(\gamma)$ by the multicover formula (2.17),

$$
D T(r, d, n)=\sum_{\substack{k \in \mathbb{Z}, k \geq 1 \\(r, d, n)=k\left(r^{\prime}, d^{\prime}, n^{\prime}\right)}} \frac{1}{k^{2}} \Omega\left(r^{\prime}, d^{\prime}, n^{\prime}\right) .
$$

For any pair $(r, \delta), \delta \in \Lambda^{\vee} / r \Lambda$, let

$$
Z_{D T}(X, r, \delta ; \tau)=\sum_{n \in \mathbb{Z}} D T(r, d, n) q^{n+d^{2} / 2 r-r},
$$

where $d \in \Lambda^{\vee}$ is an arbitrary representative of $\delta$. Again, the right hand side of the above equation depends only on the equivalence class $\delta$ of $d \bmod r \Lambda$ since the rational Donaldson-Thomas invariants are invariant under transformations (2.3) as well. In order to evaluate this series, first note that for any pair $\left(k, r^{\prime}\right)$ with $k r^{\prime}=r$ there is an injective morphism

$$
f_{r^{\prime}, k}: \Lambda^{\vee} / r^{\prime} \Lambda \rightarrow \Lambda^{\vee} / r \Lambda, \quad f_{r^{\prime}, k}\left([d]_{r^{\prime}}\right)=[k d]_{r}, \quad \text { for all } d \in \Lambda^{\vee} .
$$


Then, using the above multicover formula, the generating functional is written as

$$
\begin{aligned}
Z_{D T}(X, r, \delta ; \tau) & =\sum_{\substack{k \in \mathbb{Z}, k \geq 1 \\
r, d)=k\left(r^{\prime}, d^{\prime}\right)}} \frac{1}{k^{2}} \sum_{n^{\prime} \in \mathbb{Z}} q^{k\left(n^{\prime}+d^{\prime 2} / 2 r^{\prime}-r^{\prime}\right)} \Omega\left(r^{\prime}, d^{\prime}, n^{\prime}\right) \\
& =\sum_{\substack{k \in \mathbb{Z}, k \geq 1 \\
r=k r^{\prime}, \delta=f_{r^{\prime}, k}\left(\delta^{\prime}\right)}} \frac{1}{k^{2}} Z_{B P S}\left(X, r^{\prime}, \delta^{\prime} ; k \tau\right) .
\end{aligned}
$$

In the right hand side, $\delta^{\prime} \in \Lambda^{\vee} / r^{\prime} \Lambda$ is uniquely determined by $(k, \delta)$ since $f_{r^{\prime}, k}$ is injective.

For the next step one needs a generalization of the conjectural formula (4.17) to all pairs $(r, \delta)$, not just primitive ones. On physics grounds, the natural conjecture at this point is that (4.17) is in fact valid for all such pairs, including non-primitive ones. The main physical argument for this conjecture is based on modularity constraints. Physics arguments based on S-duality [16] or M5-brane elliptic genus [20, 15] imply that the collection of partition functions $Z_{D T}(X, r, \delta ; \tau)$, $\delta \in \Lambda^{\vee} / r \Lambda$ must be a meromorphic vector valued modular form of weight $(-1-\ell / 2)$. This vector valued modular form must take values in a finite dimensional unitary representation of the metaplectic cover of $S L(2, \mathbb{Z})$ on the $\mathbb{C}$-linear span $\mathbb{C}\left[\Lambda^{\vee} / r \Lambda\right]$.

Granting this statement, it follows that the whole generating function $Z_{D T}(X, r, \delta ; \tau)$ is completely determined by the Donaldson-Thomas invariants for primitive charges. The main point is that although $(r, \delta)$ is non-primitive, for any representative $d \in \Lambda^{\vee}$ of $\delta$, there are infinitely many values of $n \in \mathbb{Z}$ such that $\gamma=(r, d, n)$ is primitive. By the arguments of the previous section, the conjectural formula (4.16) will apply to all such values. Then the generating function $Z_{B P S}(X, r, \delta ; \tau)$ will be given by equation (4.17) for all pairs $(r, \delta)$ since the vector space of weight $(-1-\ell / 2)$ vector valued modular forms is finite dimensional. As shown below this leads to the final expression (4.20) for the partition function of rational invariants. In Section 6, it will be shown that these generating functions are indeed the coefficients of a weight $(-1-\ell / 2)$ vector valued modular form with values in the Weil representation associated to the lattice $\sqrt{r} \Lambda \subset \Lambda_{\mathbb{R}}$. Moreover, the vector space of such vector valued modular forms is indeed finite dimensional. Further confimation of this conjecture will be obtained in Section 5.3 employing a more mathematical approach.

Granting formula (4.17) for all charge vectors, one obtains

$$
Z_{D T}(r, \delta ; \tau)=\sum_{\substack{k \in \mathbb{Z}, k \geq 1 \\ r=k r^{\prime}, \delta=f_{r^{\prime}, k}\left(\delta^{\prime}\right)}} \frac{1}{k^{2}} \frac{1}{2 r^{\prime}} \sum_{s=0}^{r^{\prime}-1} e^{-\pi i s\left(d^{\prime}\right)^{2} / r^{\prime}}\left(\Delta^{-1} \widetilde{\Phi}_{\left[d^{\prime}\right]_{1}}\right)\left(\frac{k \tau+s}{r^{\prime}}\right)
$$

Now note that for any $l \in \mathbb{Z}, l \geq 1$, the $\mathbb{Q}$-valued symmetric bilinear pairing of $\Lambda^{\vee}$ induces a $\mathbb{Q} / \mathbb{Z}$-valued symmetric bilinear pairing $(,)_{l}$ on $\Lambda^{\vee} / l \Lambda$ given by

$$
\left([d]_{l},\left[d^{\prime}\right]_{l}\right)_{l}=\frac{d \cdot d^{\prime}}{l} \bmod \mathbb{Z}
$$


Moreover, since the intersection pairing on $\Lambda$ is even, there is a well defined $\mathbb{Q} / \mathbb{Z}$-valued quadratic form $\theta_{l}: \Lambda^{\vee} / l \Lambda \rightarrow \mathbb{Q} / \mathbb{Z}$

$$
\theta_{l}(\eta)=\frac{(\eta, \eta)_{l}}{2}
$$

Then the formula can be further rewritten as

$$
Z_{D T}(X, r, \delta ; \tau)=\frac{1}{2 r^{2}} \sum_{\substack{k \in \mathbb{Z}, k \geq 1 \\ r=k l, \delta=f_{l, k}(\eta)}} \sum_{s=0}^{l-1} l e^{-2 \pi i s \theta_{l}(\eta)}\left(\Delta^{-1} \widetilde{\Phi}_{[\eta]_{1}}\right)\left(\frac{k \tau+s}{l}\right),
$$

where $[\eta]_{1} \in \Lambda^{\vee} / \Lambda$ is the equivalence class of $\eta \in \Lambda^{\vee} / l \Lambda \bmod \Lambda$. This is precisely equation (1.3).

Finally, the total rank $r \geq 1$ generating function for Donaldson-Thomas invariants is obtained by summing over all $d \in \Lambda^{\vee}$. As shown in Section 2, this yields a sum of the form

$$
Z_{D T}(X, r ; \tau, \bar{\tau}, B, C)=\sum_{\delta \in \Lambda^{\vee} / \Lambda} Z_{D T}(X, r, \delta ; \tau) \Theta_{r, \delta}^{*}(\tau, \bar{\tau} ; C, B)
$$

where

$$
\Theta_{r, \delta}^{*}(\tau, \bar{\tau})=\sum_{\alpha \in \Lambda} e^{-2 \pi i \tau(d+r B+r \alpha)_{-}^{2} / 2 r-2 \pi i \bar{\tau}(d+r B+r \alpha)_{+}^{2} / 2 r+2 \pi i(d+r B+r \alpha) \cdot C} .
$$

In the right hand side of the above expression $d \in \Lambda^{\vee}$ is an arbitrary representative of $\delta$. Using the multicover formula for Donaldson-Thomas one obtains

$$
Z_{D T}(X, r ; \tau, \bar{\tau}, B, C)=\sum_{\delta \in \Lambda^{\vee} / r \Lambda} \sum_{\substack{k \in \mathbb{Z}, k \geq 1 \\ r=k r^{\prime}, \delta=f_{r^{\prime}, k}\left(\delta^{\prime}\right)}} \frac{1}{k^{2}} Z_{B P S}\left(X, r^{\prime}, \delta^{\prime} ; k \tau\right) \Theta_{r, \delta}^{*}(\tau, \bar{\tau} ; C, B) .
$$

Moreover

$$
\Theta_{r, \delta}^{*}(\tau, \bar{\tau} ; B, C)=\Theta_{r^{\prime}, \delta^{\prime}}^{*}(k \tau, k \bar{\tau} ; k C, B) .
$$

Then, using equation (4.18), one then obtains by straightforward computations

$$
\begin{aligned}
& Z_{D T}(X, r ; \tau)= \\
& \frac{1}{2 r^{2}} \sum_{\substack{k, l \in \mathbb{Z}, k, l \geq 1 \\
k l=r}} \sum_{\eta \in \Lambda^{\vee} / l \Lambda} \sum_{s=0}^{l-1} l\left(\Delta^{-1} \widetilde{\Phi}_{[\eta]_{1}}\right)\left(\frac{k \tau+s}{l}\right) \Theta_{l, \eta}^{*}(k \tau+s, k \bar{\tau}+s ; k C, B) .
\end{aligned}
$$

Next recall that there is an exact sequence of finite abelian groups

$$
0 \rightarrow \Lambda / l \Lambda \rightarrow \Lambda^{\vee} / l \Lambda \rightarrow \Lambda^{\vee} / \Lambda \rightarrow 0
$$

Given an element $\eta \in \Lambda^{\vee} / l \Lambda$ one can first sum over all classes of the form $\eta+\gamma$ with $\gamma \in \Lambda / l \Lambda$ in the right hand side of (4.21). By a simple computation, this sum turns out to be

$$
\sum_{\gamma \in \Lambda / l \Lambda} \Theta_{l, \eta+\gamma}^{*}(k \tau+s, k \bar{\tau}+s ; B, k C)=\Theta_{1,[\eta]_{1}}^{*}\left(\frac{k \tau+s}{l}, \frac{k \bar{\tau}+s}{l} ; k C+s B, l B\right),
$$


which depends only on the equivalence class $[\eta]_{1} \in \Lambda^{\vee} / \Lambda$. Therefore formula (4.21) for the partition function can be rewritten as

$$
\begin{aligned}
& Z_{D T}(X, r ; \tau)= \\
& \frac{1}{2 r^{2}} \sum_{\substack{k, l \in \mathbb{Z}, k, l \geq 1 \\
k l=r}} \sum_{\rho \in \Lambda^{\vee} / \Lambda} \sum_{s=0}^{l-1} l\left(\Delta^{-1} \widetilde{\Phi}_{\rho}\right)\left(\frac{k \tau+s}{l}\right) \Theta_{1, \rho}^{*}\left(\frac{k \tau+s}{l}, \frac{k \bar{\tau}+s}{l} ; k C+s B, l B\right) .
\end{aligned}
$$

For $B=0$ and $C=0$ one can immediately recognize this formula as an order $r$ Hecke transform of the rank 1 result, as stated in Section 1.2. For nonzero $B, C$ this formula is a Hecke transform for Jacobi forms, as discussed in more detail in [8]. For completeness, a brief definition of Hecke operators for modular forms is given below, following for example [66, Ch.4. Part.2].

Let $\mathcal{M}_{n}$ be the set of $2 \times 2$ matrices with entries in $\mathbb{Z}$, of determinant $n$, and note that there is a finite set of orbits $\Gamma_{1} \backslash \mathcal{M}_{n}$ under left multiplication by $\Gamma_{1}=P S L(2, \mathbb{Z})$. Then the order $n$ Hecke operator [66, Ch.4. Part.2] is an endomorphism of the space of the space of holomorphic modular forms of fixed weight $w$ defined by

$$
T_{n} f(\tau)=n^{w-1} \sum_{\gamma \in \Gamma_{1} \backslash \mathcal{M}_{n}}(c \tau+d)^{-w} f(\gamma \cdot \tau)
$$

where $\left(\begin{array}{ll}a & b \\ c & d\end{array}\right) \in \mathcal{M}_{n}$ is a representative of $\gamma$. The right hand side does not depend on the choice of representative. Moreover, one can prove that the above operator can be written as

$$
T_{n} f(\tau)=n^{w-1} \sum_{\substack{a, d, \in \mathbb{Z}, a, d>0, a d=n}} \sum_{b=0}^{d-1} d^{-w} f\left(\frac{a \tau+b}{d}\right) .
$$

This is a consequence of Theorem 1 in [66, Ch.4. Part.2]. The same construction applies analogously to non-holomorphic modular forms and Jacobi forms.

\section{RECURSIVE DERIVATION FROM STABLE PAIR INVARIANTS}

The goal of this section is to provide an alternative derivation for the main formula (1.3) based on the mathematical results of [24]. Using wallcrossing techniques, vertical Donaldson-Thomas invariants for nodal K3 pencils are related in loc. cit. to the stable pair invariants defined by Pandharipande and Thomas in [55. It will be shown in this section that this relation determines the Donaldson-Thomas invariants in terms of stable pair ones by a recursive algorithm. Moreover, assuming Gromov-Witten/stable pair correspondence and the multicover formula (2.17), this algorithm yields a proof of the main formula (1.3). Using the results of Maulik and Pandharipande on Gopakumar-Vafa invariants of K3 pencils, the recursive algorithm reduces the proof of the main formula to a remarkable combinatorial identity which is proven in Section 5.4 . 
5.1. Background. Let $X$ be a nonsingular projective threefold, $v \in H_{2}(X, \mathbb{Z})$ a curve class, and $n \in \mathbb{Z}$. According to [55], the moduli space of stable pairs $\operatorname{PT}_{n}(X, v)$ parametrizes the pairs $\mathcal{O}_{X} \stackrel{s}{\rightarrow} \mathcal{F}$ where $\mathcal{F}$ is a pure 1-dimensional sheaf on $X$ with $\operatorname{ch}_{2}(\mathcal{F})=v$ and $\chi(\mathcal{F})=n$ such that the cokernel of $s$ is 0 -dimensional. It was shown by the authors that $\operatorname{PT}_{n}(X, v)$ is a locally complete moduli space of complexes in the derived category, which enabled them to construct a perfect obstruction theory on $\mathrm{PT}_{n}(X, v)$. In the case where the virtual dimension of the moduli space is zero, the stable pair invariants $P_{n, v}$ are defined by taking the degree of the virtual cycle obtained from this obstruction theory.

Now suppose $X$ has a $K 3$ fibration structure $\pi: X \rightarrow C$ over a smooth projective curve $C$ with at most nodal fibers. The sublattice of vertical classes will be denoted by $H_{2}(X, \mathbb{Z})^{\pi}=\operatorname{Ker}\left(\pi_{*}\right) \subset$ $H_{2}(X, \mathbb{Z})$. Since the canonical class of $X$ is vertical, it is easy to show that the virtual dimension of the moduli space of stable pairs is zero for any $v \in H_{2}(X, \mathbb{Z})^{\pi}$ and any $n \in \mathbb{Z}$. Such stable pairs, will be called "vertical stable pairs". The vertical stable pair series of $X$ is then defined by

$$
P T^{\text {vert }}(X)=\sum_{\substack{n \in \mathbb{Z}, v \in H_{2}(X, \mathbb{Z})^{\pi}}} P_{n, v} q^{n} t^{v} .
$$

Note that in the above formula one has to sum only over nonzero, effective curve classes. This condition will be written as $v>0$.

For $X$ a smooth Calabi-Yau threefold, one of the main results of [24] relates the vertical stable invariants of $X$ to the vertical DT invariants $D T(r, v, n)$ introduced in Section 2.3, Recall that the latter are virtual counting invariants for semistable sheaves $E$ on $X$ with numerical invariants

$$
\operatorname{ch}_{0}(E)=0, \quad \operatorname{ch}_{1}(E)=r D, \quad \operatorname{ch}_{2}(E)=v, \quad \operatorname{ch}_{3}(E)=-n \operatorname{ch}_{3}\left(\mathcal{O}_{x}\right),
$$

where $x \in X$ is an arbitrary closed point. Therefore they are related to the invariants used in [24, Thm. 2] by $D T(r, v, n)=J(r, v,-n)$. Assuming a certain technical conjecture, 24, Conjecture 2.3], the authors of [24] proved the following identity [24, Thm. 2] using wall-crossing techniques:

$$
\begin{aligned}
\mathrm{PT}^{\mathrm{vert}}(X)=\prod_{r \geq 0, v>0, n \leq 0} \exp \left((-1)^{n-1} D T(r, v, n) q^{-n} t^{v}\right)^{-n+2 r} \\
\cdot \prod_{r>0, v>0, n<0} \exp \left((-1)^{n-1} D T(r, v, n) q^{n} t^{v}\right)^{-n+2 r} .
\end{aligned}
$$

In order to make contact with the set up of Sections 3 and 2.3 suppose the K3 fibration $\pi$ : $X \rightarrow C$ satisfies conditions $(a)-(d)$ in Section 3 , Then Poincaré duality yields an isomorphism $H_{2}(X, \mathbb{Z})^{\pi} \simeq \Lambda^{\vee}$, where $\Lambda$ is the polarizing lattice for the pencil $\tilde{X}$. Recall that $\Lambda$ is a rank $\ell$ sublattice of the middle cohomology lattice $\Lambda_{K 3}$ of a smooth generic K3 surface. Moreover, the natural intersection form on $\Lambda_{K 3}$ restricts to a nondegenerate symmetric bilinear form on $\Lambda$ of signature $(1, \ell-1)$. Therefore the vertical classes $v$ will be identified with dual lattice vectors $d \in \Lambda^{\vee}$. Using the given bilinear form, $\Lambda$ is identified with a sublattice of $\Lambda^{\vee}$. Furthermore the integral bilinear form on $\Lambda$ extends to a $\mathbb{Q}$-valued bilinear form on $\Lambda^{\vee}$, which will be denoted by 
$\left(d, d^{\prime}\right) \mapsto d \cdot d^{\prime}$. By construction, $\alpha \cdot d=d \cdot \alpha=d(\alpha) \in \mathbb{Z}$ for any any $\alpha \in \Lambda, d \in \Lambda^{\vee}$. Finally note that for any such pair $(\alpha, d)$, the lattice product $d \cdot \alpha$ is the same as the intersection product of $\alpha$ and $d$ viewed as homology classes on $X$ as explained above. Therefore no notational distinction between such cases will be made in this section.

The next subsection will provide a recursive method for computing the invariants $D T(r, d, n)$ on the right hand side of (5.1) directly from the partition function $\operatorname{PT}(X)$ of $P_{n, d}$ invariants on the left hand side.

5.2. A recursive algorithm for $D T(r, d, n)$. The recursive algorithm for the invariants $\{D T(r, d, n)\}$ is based on the following two key properties.

1. $D T(r, d, n) \neq 0$ implies either $r=0$, or

$$
d^{2}+2 r n \geq 0 .
$$

2. For any $(r, d, n)$ there is an identity

$$
D T(r, d, n)=D T\left(r, d+r \alpha, n-r \alpha^{2} / 2-r d \cdot \alpha\right),
$$

where $\alpha$ is a divisor class of $X$ restricted to the fiber of $\pi$.

The first property follows from the Bogomolov inequality. In more detail, the proof proceeds in several steps, as follows. Suppose first $E$ is a stable sheaf on $X$ supported on a smooth $K 3$ fiber $\iota_{p}: X_{p} \hookrightarrow X$, with invariants $(r, d, n)$. Then $E$ is the extension by zero of a stable sheaf $F$ on $X_{p}$ with invariants

$$
\operatorname{rk}(F)=r, \quad c_{1}(F)=\beta, \quad c_{2}(F)=k
$$

such that

$$
\iota_{p *}(\beta)=d, \quad k-\frac{\beta^{2}}{2}=n .
$$

If $r>0, F$ must be torsion free and the Bogomolov inequality reads

$$
k-\frac{r-1}{2 r} \beta^{2} \geq 0
$$

which is equivalent to

$$
\beta^{2}+2 r n \geq 0 .
$$

As explained in Section 3.3, the algebraic Hodge theorem yields a second inequality,

$$
\beta^{2} \leq d^{2} .
$$

This proves inequality (5.2).

Next suppose $E$ is a stable sheaf scheme theoretically supported on a nodal fiber $\iota_{\sigma}: X_{\sigma} \rightarrow X$. In that case inequality (5.2) is proven by analogy with Lemmas 4.3 and 4.4 in [17]. Finally, suppose $E$ is semistable. Then inequality (5.2) is determined by from the above results for stable shaves using a Jordan-Hölder filtration by analogy with Lemma 4.5 in [17]. 
As noted in Section 2.3, the second property follows from the observation that the tensor product by the line bundle $\mathcal{O}_{X}(\alpha)$ yields an isomorphism of moduli stacks of semistable sheaves for any $\alpha \in \Lambda \subset \operatorname{Pic}(X)$.

In order to construct the recursive algorithm for $D T(r, d, n)$, note that equation (5.1) yields

$$
\begin{aligned}
& \log (\mathrm{PT}(X))=\sum_{r \geq 0, d>0, n \leq 0}(-1)^{n-1}(-n+2 r) D T(r, d, n) q^{-n} t^{d} \\
& +\sum_{r>0, d>0, n<0}(-1)^{n-1}(-n+2 r) D T(r, d, n) q^{n} t^{d} .
\end{aligned}
$$

Then one proceeds inductively by $r \in \mathbb{Z}, r \geq 0$.

Step 1. $r=0$. According to property (2) above, $D T(0, d, n)=D T(0, d, n-d \cdot \alpha)$ for any divisor $H \in \Lambda \subset \operatorname{Pic}(X)$. Now note that if $H$ is sufficiently relatively ample with respect to the projection map $\pi: X \rightarrow \mathbb{P}^{1}$, the coefficient of $q^{-n+d \cdot \alpha} t^{d}$ on the right hand side of (5.3) does not contain any $r>0$ terms. Indeed if otherwise it does, then inequality (5.2) implies

$$
d^{2}+2 r(n-d \cdot \alpha) \geq 0
$$

Since $d>0$, this leads to a contradiction for sufficiently relatively ample $\alpha$, keeping $(d, n)$ fixed. Therefore the invariant $D T(0, d, n)$ is determined by the coefficient of $q^{-n+d \cdot \alpha} t^{d}$ for $\alpha \gg 0$ in the left hand side of (5.3).

Step 2. Induction on $r>0$ and computation of $D T(r, d, n)$ invariants: Now apply the induction on $r$. Suppose that $r>0$ and every $D T\left(r^{\prime}, d, n\right)$ is expressed with respect to the stable pair invariants for $r^{\prime}<r$. By property (2) above,

$$
D T(r, d, n)=D T\left(r, d+r \alpha, n-r \alpha^{2} / 2-d \cdot \alpha\right)
$$

for any $\alpha \in \Lambda$. Let $\gamma=(r, d, n)$ and $N(\gamma, \alpha)=-n+r \alpha^{2} / 2+d \cdot \alpha$. Again, the key observation is that if $\alpha$ is sufficiently relatively ample, the coefficient of $q^{N(\gamma, \alpha)} t^{d+r \alpha}$ in the right hand side of (5.3) does not contain any invariants

$$
D T(R, d+r \alpha,-N(\gamma, \alpha))
$$

with $R>r$. Indeed if it does otherwise, then property (2) implies that

$$
(d+r \alpha)^{2}+2 R\left(n-r \alpha^{2} / 2-d \cdot \alpha\right) \geq 0
$$

Keeping $(r, d, n)$ fixed, this leads to a contradiction for any $R>r$, if $\alpha$ is sufficiently relatively ample. Therefore for such divisors $\alpha$, the sum (5.3)

$$
(-1)^{N(\gamma, \alpha)-1} \sum_{s=0}^{r}(N(\gamma, \alpha)+2 s) D T(s, d+r \alpha,-N(\gamma, \alpha))
$$

is identified with the coefficient of $q^{N(\gamma, \alpha)} t^{d+r \alpha}$ in the left hand side of (5.3). Using the induction, one can then express $D T(r, d, n)$ in terms of stable pair invariants. 
5.3. Vertical Donaldson-Thomas invariants from Gopakumar-Vafa invariants. The goal of this section is to show that the conjectural formula (4.16) follows from the above recursive algorithm assuming the Gromov-Witten/stable pair correspondence to hold in the current setup. If this is the case, the vertical stable pair partition function can be written in terms of vertical Gopakumar-Vafa invariants, which have been computed in [46]:

$$
\log (\operatorname{PT}(X))=\sum_{g \geq 0} \sum_{\substack{d \in \Lambda^{\vee} \\ d>0}} \sum_{k=1}^{\infty} \frac{(-1)^{g-1}}{k} n_{g, d}(X)\left(\frac{\left(1-(-q)^{k}\right)^{2}}{(-q)^{k}}\right)^{g-1} t^{k d}
$$

where $n_{g, d}(X)$ are the vertical Gopakumar-Vafa invariants of $X$. According to [46, Thm. 1],

$$
n_{g, d}(X)=\frac{1}{2} \sum_{h=g}^{\infty} r_{g, h} \widetilde{N L}_{h, d}
$$

where $r_{g, h}$ are the local Gopakumar-Vafa invariants of a smooth K3 surface and $\widetilde{N L}_{h, d}$ are the Noether-Lefschetz numbers of the K3 pencil $\tilde{\pi}: \widetilde{X} \rightarrow \Sigma$ constructed in Section 3 , An explicit conjecture for the invariants $r_{g, h}$ was formulated by Katz, Klemm and Vafa [34, and proven by Pandharipande and Thomas [54]. This reads

$$
\sum_{g \geq 0} \sum_{h \geq 0}(-1)^{g} r_{g, h}\left(\frac{(1-y)^{2}}{y}\right)^{2 g} q^{h}=\prod_{n \geq 1} \frac{1}{\left(1-q^{n}\right)^{20}\left(1-y q^{n}\right)^{2}\left(1-y^{-1} q^{n}\right)^{2}} .
$$

The above formula implies in particular that $r_{g, h}=0$ for $g>h$.

According the inductive step in the previous section, for a given triple $\gamma=(r, d, n)$, one has to choose a sufficiently relatively ample divisor $\alpha \in \Lambda \subset \operatorname{Pic}(X)$. Then the linear combination

$$
(-1)^{N(\gamma, \alpha)-1} \sum_{s=0}^{r}(N(\gamma, \alpha)+2 s) D T(s, d+r \alpha,-N(\gamma, \alpha))
$$

is identified with the the coefficient $L_{N(\gamma, \alpha), d+r \alpha}$ of $q^{N(\gamma, \alpha)} t^{d+r \alpha}$ in (5.4), and $N(\gamma, \alpha)=-n+$ $r \alpha^{2} / 2+d \cdot \alpha$. Note that $L_{N(\gamma, \alpha), d+r \alpha}$ can be written as

$$
L_{N(\gamma, \alpha), d+r \alpha}=L_{N(\gamma, \alpha), d+r \alpha}^{0}+L_{N, d+r \alpha}^{\geq 2}
$$

where $L_{N(\gamma, \alpha), d+r \alpha}^{0}$ is the contribution of genus $g=0$ terms while $L_{N, d+r \alpha}^{\geq 2}$ encodes the terms with $g \geq 2$. Genus $g=1$ terms are obviously absent for sufficiently large $N(\gamma, \alpha)>0$. Expanding the right hand side of (5.4) in powers of $q$ yields

$$
L_{N(\gamma, \alpha), d+r \alpha}^{0}=(-1)^{N(\gamma, \alpha)-1} N(\gamma, \alpha) \sum_{\substack{k \in \mathbb{Z}, k \geq 1 \\ k \mid(d+r \alpha, N(\gamma, \alpha))}} \frac{1}{k^{2}} n_{0,(d+r \alpha) / k}
$$


and

$$
L_{N(\gamma, \alpha), d+r \alpha}^{\geq 2}=(-1)^{N(\gamma, \alpha)} \sum_{\substack{k \in \mathbb{Z}, k \geq 1 \\
k \mid(d+r \alpha, N(\gamma, \alpha))}} \sum_{g \geq 1+N(\gamma, \alpha) / k} \frac{(-1)^{N(\gamma, \alpha) / k}}{k} n_{g,(d+r \alpha) / k}\left(\begin{array}{c}
2 g-2 \\
g-1-N(\gamma, \alpha) / k
\end{array}\right) .
$$

Note that for $r=0$, the expression (5.7) reduces to

$$
(-1)^{N(\gamma, \alpha)-1} N(\gamma, \alpha) D T(0, d+r \alpha,-N(\gamma, \alpha))
$$

Using the multicover formula for Donaldson-Thomas invariants, this is further equal to

$$
(-1)^{N(\gamma, \alpha)-1} N(\gamma, \alpha) \sum_{\substack{k \in \mathbb{Z}, k \geq 1 \\ k \mid(d+r \alpha, N(\gamma, \alpha))}} \frac{1}{k^{2}} \Omega(0,(d+r \alpha) / k,-N(\gamma, \alpha) / k) .
$$

Moreover, as conjectured in [33], for any $v \in H^{2}(X, \mathbb{Z})$ and any $N \in \mathbb{Z}$, one has $\Omega(0, v, N)=n_{0, v}$. Therefore $L_{N(\gamma, \alpha), d+r \alpha}^{0}$ in equation (5.8) equals the $s=0$ term in (5.7).

Next, using (5.5), the higher genus contributions $L_{N(\gamma, \alpha), d+r \alpha}^{\geq 2}$ can be written in terms of the local invariants $r_{g, h}$ and Noether-Lefschetz numbers. Note that $\widetilde{N L}_{h, d}=0$ for $h>d^{2} / 2+1$ and $r_{g, h}=0$ for $g>h$. This implies that

$$
\frac{N(\gamma, \alpha)}{k}+1 \leq g \leq h \leq \frac{(d+r \alpha)^{2}}{2 k^{2}}+1
$$

for any $r_{g, h}$ occuring by substitution in the right hand side of equation (5.9). Then equations (5.5), (5.9), yield

$$
\begin{aligned}
& L_{N(\gamma, \alpha), d+r \alpha}^{\geq 2}=\sum_{\substack{k \in \mathbb{Z}, k \geq 1 \\
k \mid(d+r \alpha, N(\gamma, \alpha))}} \frac{(-1)^{(k+1) N(\gamma, \alpha) / k}}{2 k} \\
& \sum_{\substack{h \in \mathbb{Z} \\
N(\gamma, \alpha) / k+1 \leq h \leq(d+r \alpha)^{2} / 2 k^{2}+1}} \widetilde{N L}_{h,(d+r \alpha) / k} \sum_{g=1+N(\gamma, \alpha) / k}^{h} r_{g, h}\left(\begin{array}{c}
2 g-2 \\
g-1-N(\gamma, \alpha) / k
\end{array}\right) .
\end{aligned}
$$

At the same time, using the multicover formula for Donaldson-Thomas invariants, the $s \geq 1$ part of the sum in (5.7) becomes

$$
\begin{gathered}
(-1)^{N(\gamma, \alpha)-1} \sum_{s=0}^{r}(N(\gamma, \alpha)+2 s) \sum_{\substack{k \in \mathbb{Z}, k \geq 1 \\
k \mid(s, d+r \alpha, N(\gamma, \alpha))}} \frac{1}{k^{2}} \Omega(s / k,(d+r \alpha) / k,-N(\gamma, \alpha) / k)= \\
(-1)^{N(\gamma, \alpha)-1} \sum_{\substack{k \in \mathbb{Z}, k \geq 1 \\
k \mid(d+r \alpha, N(\gamma, \alpha))}} \sum_{\substack{u \in \mathbb{Z} \\
1 \leq u \leq r / k}} \frac{1}{k}\left(\frac{N(\gamma, \alpha)}{k}+2 u\right) \Omega(u,(d+r \alpha) / k,-N(\gamma, \alpha) / k) .
\end{gathered}
$$


Now recall the conjectural formula (4.16) for the vertical D4-D2-D0 degeneracies. For any triple $\gamma=(r, d, n), r \geq 1$, one has

$$
\Omega(\gamma)=\frac{1}{2} \sum_{\substack{h \in \mathbb{Z} \\ r(r-n) \leq h \leq d^{2} / 2+1}} c(r(n-r)+h) \widetilde{N L}_{h, d}
$$

where

$$
\sum_{n \geq 0} c(n) q^{n}=\frac{1}{\prod_{n \geq 1}\left(1-q^{n}\right)^{24}}
$$

Note that by comparison with equation (5.5), it follows that $c(n)=r_{0, n}$ for any $n \geq 0$. Using equation (5.13), one obtains

$$
\Omega(u,(d+r \alpha) / k,-N(\gamma, \alpha) / k)=\frac{1}{2} \sum_{\substack{h \in \mathbb{Z} \\ u(u+N(\gamma, \alpha) / k) \leq h \leq(d+r \alpha)^{2} / 2 k^{2}+1}} c(h-u(u+N(\gamma, \alpha) / k)) \widetilde{N L}_{h,(d+r \alpha) / k}
$$

Next note that for $u \geq 1$ the inequality

$$
u\left(u+\frac{N(r, \alpha)}{k}\right) \leq \frac{(d+r \alpha)^{2}}{2 k^{2}}+1
$$

is equivalent to

$$
u \leq-\frac{N(\gamma, \alpha)}{2 k}+\frac{1}{2 k}\left(N(\gamma, \alpha)^{2}+2(d+r \alpha)^{2}+4 k^{2}\right)^{1 / 2}
$$

Moreover note that one must have $k \leq r$ for all nonzero terms in the right hand side of (5.12) since $u \geq 1$. Keeping in mind that $N(\gamma, \alpha)=-n+r \alpha^{2} / 2+d \cdot \alpha$, a straightforward series expansion shows that for fixed $\gamma=(r, d, n)$ there exists a sufficiently relatively ample $\alpha$ such that inequality (5.15) is equivalent to $u \leq r / k$. The latter is in turn precisely the upper bound on $u$ in the sum (5.12).

At the same time, since $u \geq 1$, for any nonzero term in the right hand side of equation (5.14) one must clearly have

$$
\frac{N(\gamma, \alpha)}{k}+1 \leq h \leq \frac{(d+r \alpha)^{2}}{2 k^{2}}+1 .
$$

Therefore expression (5.12) can be further written as

$$
\begin{gathered}
(-1)^{N(\gamma, \alpha)-1} \sum_{\substack{k \in \mathbb{Z}, k \geq 1 \\
k \mid(d+r \alpha, N(\gamma, \alpha))}} \frac{1}{2 k} \sum_{\substack{h \in \mathbb{Z} \\
N(\gamma, \alpha) / k+1 \leq h \leq(d+r \alpha)^{2} / 2 k^{2}+1}} \widetilde{N L}_{h,(d+r \alpha) / k} \\
\sum_{\substack{u \in \mathbb{Z}, u \geq 1 \\
u(u+N(\gamma, \alpha) / k) \leq h}}\left(\frac{N(\gamma, \alpha)}{k}+2 u\right) r_{0, h-u(u+N(\gamma, \alpha) / k)} .
\end{gathered}
$$


In order to facilitate comparison with (5.11), note that the latter reads

$$
\begin{aligned}
& L_{N(\gamma, \alpha), d+r \alpha}^{\geq 2}=\sum_{\substack{k \in \mathbb{Z}, k \geq 1 \\
k \mid(d+r \alpha, N(\gamma, \alpha))}} \frac{(-1)^{(k+1) N(\gamma, \alpha) / k}}{2 k} \\
& \sum_{\substack{h \in \mathbb{Z} \\
N(\gamma, \alpha) / k+1 \leq h \leq(d+r \alpha)^{2} / 2 k^{2}+1}} \widetilde{N L}_{h,(d+r \alpha) / k} \sum_{g=1+N(\gamma, \alpha) / k}^{h} r_{g, h}\left(\begin{array}{c}
2 g-2 \\
g-1-N(\gamma, \alpha) / k
\end{array}\right) .
\end{aligned}
$$

Therefore it follows that the two expressions are in agreement provided that the following identity holds for any $h, n \in \mathbb{Z}, n \geq 1, h \geq n+1$ :

$$
\sum_{g=n+1}^{h} r_{g, h}\left(\begin{array}{c}
2 g-2 \\
g-n-1
\end{array}\right)=(-1)^{n-1} \sum_{\substack{s \in \mathbb{Z}, s \geq 1 \\
s(s+n) \leq h}}(n+2 s) r_{0, h-s(s+n)} .
$$

This is rigorously proven in the next subsection.

In conclusion, formula (4.16) derived in Section 4.2 on physics grounds indeed follows from the wallcrossing indentity (5.1). The above mathematical derivation holds for all charge vectors, not just primitive ones, confirming the string theoretic conjecture made in Section 4.4 below equation (4.19).

5.4. Proof of the combinatorial identity. Identity (5.17) is equivalent to

$$
\sum_{s=1}^{\infty}(n+2 s) r_{0, h-s(s+n)}=(-1)^{n-1} \sum_{g=n+1}^{h}\left(\begin{array}{c}
2 g-2 \\
g-n-1
\end{array}\right) r_{g, h} .
$$

for any $n \in \mathbb{Z}, 0 \leq n \leq|h|$, provided that, by convention, $r_{0, k}=0$ for $k<0$.

In order to prove this, first recall the relevant statements of [46]. The $r_{g, h}$ are given by

$$
\sum_{g \geq 0} \sum_{h \geq 0}(-1)^{g} r_{g, h}\left(y^{\frac{1}{2}}-y^{-\frac{1}{2}}\right)^{2 g} q^{h}=\prod_{n \geq 0} \frac{1}{\left(1-q^{n}\right)^{20}\left(1-y q^{n}\right)^{2}\left(1-y^{-1} q^{n}\right)^{2}}
$$

where the right-hand side is a meromorphic function on $\mathbb{H} \times \mathbb{C}$ and this identity only holds if $|q y|<1$ and $\left|y^{-1} q\right|<1$. Suppose in addition $|y|<1$. Then this formula has two consequences, first $r_{g, h}=0$ if $g>h$ and also in the specialization $y=1$ it reduces to

$$
\sum_{h \geq 0} r_{0, h} q^{h}=\prod_{n \geq 1} \frac{1}{\left(1-q^{n}\right)^{24}} .
$$

Secondly we recall the meromorphic index minus two Jacobi form $\Phi_{2}(y, q)$ of [10], actually its rescaled version, rescaled by $\eta(q)^{-18}$ is

$$
\frac{\Phi_{2}(y, q)}{\eta(q)^{18}}=-\frac{1}{q\left(y^{\frac{1}{2}}-y^{-\frac{1}{2}}\right)^{2}} \prod_{n \geq 0} \frac{1}{\left(1-q^{n}\right)^{20}\left(1-y q^{n}\right)^{2}\left(1-y^{-1} q^{n}\right)^{2}} .
$$


The main result of that article are Fourier expansions of meromorphic negative index Jacobi forms and in that case Theorem 1.3 of [10] yields

$$
\frac{\Phi_{2}(y, q)}{\eta(q)^{18}}=-\frac{1}{\eta(q)^{24}} \sum_{n \in \mathbb{Z}} \frac{(2 n+1) y q^{n(n+1)}}{\left(1-y q^{n}\right)}+\frac{y^{2} q^{n(n+2)}}{\left(1-y q^{n}\right)^{2}} .
$$

Define the Fourier coefficients $a_{n, h}$ as

$$
\frac{\Phi_{2}(y, q)}{\eta(q)^{18}}=\sum_{\substack{n \in \mathbb{Z} \\ h \geq-1}} a_{n, h} y^{n} q^{h}
$$

where $|q y|<1$ and $\left|y^{-1} q\right|<1$ and also $|y|<1$. These coefficients can be computed in two fashions and the comparison will yield the theorem. First, using the formula of [46] we get

$$
\begin{aligned}
\frac{\Phi_{2}(y, q)}{\eta(q)^{18}} & =-\frac{1}{q\left(y^{\frac{1}{2}}-y^{-\frac{1}{2}}\right)^{2}} \prod_{n \geq 0} \frac{1}{\left(1-q^{n}\right)^{20}\left(1-y q^{n}\right)^{2}\left(1-y^{-1} q^{n}\right)^{2}} \\
& =-\frac{1}{q\left(y^{\frac{1}{2}}-y^{-\frac{1}{2}}\right)^{2}} \sum_{h \geq 0} \sum_{g=0}^{h}(-1)^{g} r_{g, h}\left(y^{\frac{1}{2}}-y^{-\frac{1}{2}}\right)^{2 g} q^{h} \\
& =-\sum_{h \geq-1} \sum_{g=0}^{h+1}(-1)^{g} r_{g, h+1}\left(y^{\frac{1}{2}}-y^{-\frac{1}{2}}\right)^{2(g-1)} q^{h} \\
& =-\sum_{h \geq-1} r_{0, h+1}\left(y^{\frac{1}{2}}-y^{-\frac{1}{2}}\right)^{-2} q^{h}-\sum_{h \geq-1} \sum_{g=1}^{h+1}(-1)^{g} r_{g, h+1}\left(y^{\frac{1}{2}}-y^{-\frac{1}{2}}\right)^{2(g-1)} q^{h} \\
& =-\sum_{h \geq-1} \sum_{n=0}^{\infty} r_{0, h+1} n y^{n} q^{h}-\sum_{h \geq-1} \sum_{g=1}^{h+1}(-1)^{g+k} r_{g, h+1}\left(\begin{array}{c}
2(g-1) \\
k
\end{array}\right) y^{g-k-1} q^{h} .
\end{aligned}
$$

In the last equality we have expanded $\left(y^{\frac{1}{2}}-y^{-\frac{1}{2}}\right)^{-2}$ in the domain $|y|<1$ and used the binomial formula for $\left(y^{\frac{1}{2}}-y^{-\frac{1}{2}}\right)^{2(g-1)}$. It follows that

$$
-a_{n, h}=n r_{0, h+1}+(-1)^{n+1} \sum_{g=1}^{h+1}(-1)^{g+k} r_{g, h+1}\left(\begin{array}{c}
2(g-1) \\
g-n-1
\end{array}\right) .
$$


Second, we will compute these coefficients using Theorem 1.3 of [10] together with the expression of $\eta(q)^{-24}$ in terms of the $r_{0, h}$, namely

$$
\begin{aligned}
\frac{\Phi_{2}(y, q)}{\eta(q)^{18}} & =-\frac{1}{\eta(q)^{24}} \sum_{n \in \mathbb{Z}} \frac{(2 n+1) y q^{n(n+1)}}{\left(1-y q^{n}\right)}+\frac{y^{2} q^{n(n+2)}}{\left(1-y q^{n}\right)^{2}} \\
& =-\sum_{h \geq 0} r_{0, h} q^{h} \sum_{n \in \mathbb{Z}} \frac{(2 n+1) y q^{n(n+1)}}{\left(1-y q^{n}\right)}+\frac{y^{2} q^{n(n+2)}}{\left(1-y q^{n}\right)^{2}} \\
& =-\sum_{h \geq 0} r_{0, h} q^{h} \sum_{n \in \mathbb{Z}} \sum_{m=0}^{\infty}(2 n+1) y^{m+1} q^{n(n+m+1)}+(m+1) y^{m+2} q^{n(n+m+2)}
\end{aligned}
$$

and hence the Fourier coefficients are

$$
-a_{n, h}=\sum_{s=0}^{\infty}(2 s+n) r_{0, h+1-s(s+n)}=n r_{0, h+1}+\sum_{s=1}^{\infty}(2 s+n) r_{0, h+1-s(s+n)} .
$$

So that comparing $a_{n, h-1}$ in (5.19) and (5.20) yields identity (5.18).

\section{Modularity of Partition FunCtions}

The goal of this section is to summarize the main modularity results obtained in this paper.

6.1. The statement. In order to fix ideas and notation, the following is a brief self-contained presentation of the construction carried in the previous sections.

- Let $\Lambda$ be a rank $\ell \geq 1$ lattice equipped with an integral even nondegenerate symmetric bilinear form of signature $(1, \ell-1)$ written as $\left(\alpha, \alpha^{\prime}\right) \mapsto \alpha \cdot \alpha^{\prime}$. This form will be often referred to as the intersection form on $\Lambda$ because of its geometric origin. Let $\left(v_{i}\right)_{1 \leq i \leq \ell}$ be a basis in $\Lambda$. The matrix $\left(v_{i} \cdot v_{j}\right)$ will be denoted by $M=\left(M_{i j}\right)$. Let $m=|\operatorname{det}(M)|$.

- Let $\Lambda^{\vee}=\operatorname{Hom}_{\mathbb{Z}}(\Lambda, \mathbb{Z})$ be the dual lattice. Using the bilinear form, $\Lambda$ is identified with a sublattice of $\Lambda^{\vee}$. For any $r \in \mathbb{Z}, r \geq 1$, the quotient $\Lambda^{\vee} / r \Lambda$ is denoted by $G_{r}$. The equivalence class of an element $d \in \Lambda^{\vee}$ in $G_{r}$ will be denoted by $[d]_{r}$. Furthermore for any $r \geq 1$ there is a natural projection $G_{r} \rightarrow G_{1}$. The image of an element $\delta \in G_{r}$ in $G_{1}$ will be denoted by $[\delta]_{1}$.

- Note that $\Lambda^{\vee} \subset \Lambda_{\mathbb{Q}}$ and there is a $\mathbb{Q}$-valued nondegenerate symmetric bilinear form pairing on $\Lambda^{\vee}$ induced by the given intersection form on $\Lambda$. In terms of the dual basis $\left(\breve{v}^{i}\right)_{1 \leq i \leq \ell}$ this pairing is given by $\left(\check{v}^{i}, \check{v}^{j}\right) \mapsto\left(M^{-1}\right)_{i j}$. Moreover, for any $l \in \mathbb{Z}, l \geq 1$, the induced form descends to a $\mathbb{Q} / \mathbb{Z}$-valued nondegenerate symmetric bilinear form $(,)_{l}$ on $G_{l}$ given by

$$
\left([d]_{l},\left[d^{\prime}\right]_{l}\right)_{l}=\frac{d \cdot d^{\prime}}{l} \bmod \mathbb{Z}
$$

In particular one obtains a $\mathbb{Q} / \mathbb{Z}$-valued quadratic form $\theta_{l}: G_{l} \rightarrow \mathbb{Q} / \mathbb{Z}$,

$$
\theta_{l}(\delta)=\frac{(\delta, \delta)_{l}}{2}
$$

for all $\delta \in G_{l}$. 
- As shown by Weil in [67, the above data determines a representation $\rho_{\Lambda}: M p(2, \mathbb{Z}) \rightarrow$ $\operatorname{End}\left(\mathbb{C}\left[G_{1}\right]\right)$ of the metaplectic group $M p(2, \mathbb{Z})$. The detailed construction is presented in Appendix $\mathrm{A}$ for completeness. For the purposes of this section, note that for a fixed $\tau$ in the upper half plane, the metaplectic group consists of pairs $(\gamma, \sqrt{c \tau+d})$ where $\gamma=\left(\begin{array}{ll}a & b \\ c & d\end{array}\right) \in$ $S L(2, \mathbb{Z})$ and is generated by $\widetilde{T}=(T, 1)$ and $\widetilde{S}=(S, \sqrt{\tau})$. The Weil representation $\rho_{\Lambda}$ is given by

$$
\rho_{\Lambda}(\widetilde{T})\left(e_{\delta}\right)=e^{2 \pi i \theta_{1}(\delta)} e_{\delta}, \quad \rho_{\Lambda}(\widetilde{S})\left(e_{\delta}\right)=\frac{e^{\pi i(\ell-2) / 4}}{\sqrt{m}} \sum_{\delta^{\prime} \in G_{1}} e^{-2 \pi i\left(\delta, \delta^{\prime}\right) 1} e_{\delta^{\prime}},
$$

where $\left(e_{\delta}\right)_{\delta \in G_{1}}$ is a basis of $\mathbb{C}\left[G_{1}\right]$. Note that this is a unitary symmetric representation, hence the dual representation $\rho_{\Lambda}^{*}$ is naturally isomorphic to its complex conjugate. This identification will be implicit throughout this section.

- A scaled form of the Weil representation will be needed below. Namely for any $r \in \mathbb{Z}$, $r \geq 1$, let $\widetilde{\Lambda} \subset \Lambda_{\mathbb{R}}=\Lambda \otimes_{\mathbb{Z}} \mathbb{R}$ be the sublattice

$$
\tilde{\Lambda}=\{\sqrt{r} \alpha \mid \alpha \in \Lambda\} .
$$

Clearly, the intersection form on $\Lambda$ induces an intersection form on $\widetilde{\Lambda}$. The dual lattice $\widetilde{\Lambda}^{\vee} \subset \Lambda_{\mathbb{R}}$ is naturally identified with the sublattice

$$
\widetilde{\Lambda}^{\vee}=\left\{d / \sqrt{r} \mid d \in \Lambda^{\vee}\right\} .
$$

Then note that there is a lattice isomorphism

$$
f: \Lambda \stackrel{\sim}{\longrightarrow} \tilde{\Lambda}, \quad f(\alpha)=\sqrt{r} \alpha
$$

which yields an isomorphism of finite abelian groups

$$
\phi: G_{r} \stackrel{\sim}{\longrightarrow} \widetilde{\Lambda}^{\vee} / \widetilde{\Lambda}, \quad \phi\left([d]_{r}\right)=[d / \sqrt{r}]_{1} .
$$

Using this isomorphism, the Weil representation associated to $\widetilde{\Lambda}$ with the induced bilinear form yields a representation $\rho_{\Lambda, r}: M p(2, \mathbb{Z}) \rightarrow \operatorname{End}\left(\mathbb{C}\left[G_{r}\right]\right)$ given by

$$
\rho_{\Lambda, r}(\widetilde{T})\left(e_{\delta}\right)=e^{2 \pi i \theta_{r}(\delta)} e_{\delta}, \quad \rho_{\Lambda, r}(\widetilde{S})\left(e_{\delta}\right)=\frac{e^{\pi i(\ell-2) / 4}}{r^{\ell / 2} \sqrt{m}} \sum_{\delta^{\prime} \in G_{r}} e^{-2 \pi i\left(\delta, \delta^{\prime}\right)_{r}} e_{\delta^{\prime}}
$$

where $\left(e_{\delta}\right)_{\delta \in G_{r}}$ is a basis of $\mathbb{C}\left[G_{r}\right]$.

- For completeness recall some basic facts on vector valued modular forms, also needed below. Given a representation $\rho: M p(2, \mathbb{Z}) \rightarrow \operatorname{End}(V)$, with $V$ a finite dimensional complex vector space, a weight $\left(w_{+}, w_{-}\right)$vector valued modular form of type $\rho$ is a $V$-valued function $\Phi(\tau, \bar{\tau})$ defined on the upper half plane such that

$$
\Phi(\gamma \cdot \tau, \gamma \cdot \bar{\tau})=(c \tau+d)^{w_{+}}(c \bar{\tau}+d)^{w_{-}} \rho(\gamma, \sqrt{c \tau+d}) \Phi(\tau, \bar{\tau})
$$


for any $\gamma=\left(\begin{array}{ll}a & b \\ c & d\end{array}\right) \in S L(2, \mathbb{Z})$, where, as usual, $\gamma \cdot \tau=\frac{a \tau+b}{c \tau+d}$. An important class of examples to be used in the construction below are the Siegel theta functions constructed in [6, Thm. 4.1].

- For any $r \in \mathbb{Z}, r \geq 1$ and for any $\delta \in G_{r}$ there is a partition function for vertical DonaldsonThomas invariants of a $\Lambda$-polarized K3 pencil given in equation (4.22). This formula is written in terms of a vector valued modular form $\Delta^{-1}(q) \widetilde{\Phi}(q)$ with values in the Weil representation of $\Lambda$. Since the geometric details are not important for modularity questions, it will be convenient to consider the following abstract variant of this construction.

Let $\Psi(\tau)=\sum_{\delta \in G_{1}} \Psi_{\delta}(\tau) e_{\delta}$ be a weight $w=-1-\ell / 2$ holomorphic vector valued modular form of type $\rho_{\Lambda}$. Note that for any $k, l \in \mathbb{Z}, k, l \geq 1$, there is an injective morphism of abelian groups

$$
f_{l, k}: G_{l} \rightarrow G_{k l}, \quad f_{l, k}\left([d]_{l}\right)=[k d]_{k l}
$$

for any $d \in \Lambda^{\vee}$. Hence any $\delta \in \operatorname{Im}\left(f_{l, k}\right)$ determines a unique element $\eta=f_{l, k}^{-1}(\delta) \in G_{l}$. Then for any $r \in \mathbb{Z}, r \geq 1$ and for any $\delta \in G_{r}$ let

$$
Z_{r, \delta}(\tau)=\frac{1}{2 r^{2}} \sum_{\substack{k, l \in \mathbb{Z}, k, l \geq 1 \\ r=k l, \delta=f_{l, k}(\eta)}} \sum_{s=0}^{l-1} l e^{-2 \pi i s \theta_{l}(\eta)} \Psi_{[\eta]_{1}}\left(\frac{k \tau+s}{l}\right) .
$$

where $[\eta]_{1} \in G_{1}$ denotes the projection of $\eta \in G_{l}$ onto $G_{1}$.

In this framework, the main modularity result obtained in this paper reads:

For any $r \in \mathbb{Z}, r \geq 1$, the vector valued series

$$
Z_{r}(\tau)=\sum_{\delta \in G_{r}} Z_{r, \delta}(\tau) e_{\delta}
$$

is a holomorphic vector valued modular form of weight $(-1-\ell / 2)$ and type $\rho_{\Lambda, r}$.

A quick proof of this statement follows from the identification of the rank $r$ partition function (1.4) with a Hecke transform of a Jacobi form. The above claim follows from the observation that the series $\Theta_{r, \delta}^{*}(\tau, \bar{\tau}, C, B)$ are in fact complex conjugates of Siegel Jacobi functions for the lattice $\widetilde{\Lambda}=\sqrt{r} \Lambda \subset \Lambda_{\mathbb{R}}$. In more detail, using the isomorphism (6.3),

$$
\Theta_{r, \delta}^{*}(\tau, \bar{\tau}, C, B)=\sum_{\tilde{\alpha} \in \tilde{\Lambda}} e^{-2 \pi i \bar{\tau}(\tilde{d}+\tilde{\alpha}+\tilde{B} / 2)_{-}^{2} / 2-2 \pi i \tau(\tilde{d}+\tilde{\alpha}+\tilde{B} / 2)_{+}^{2} / 2+2 \pi i(\tilde{d}+\tilde{\alpha}+\tilde{B} / 2) \cdot \tilde{C}},
$$

where $\tilde{d}=d / \sqrt{r}, \tilde{B}=\sqrt{r} B, \tilde{C}=\sqrt{r} C$. Therefore they form a vector valued modular form of weight $((\ell-1) / 2,1 / 2)$ with values in the complex conjugate representation $\rho_{\Lambda, r}^{*}$, and they satisfy the required linearly independence conditions. For the skeptical reader, a second proof of the above statement will be provided below in a separate subsection. 
To conclude this section, note that this modularity statement yields the finite dimensionality result needed in the derivation of final expression (4.20) for the partition function. In order to employ modularity arguments, one has to know that the vector space of all possible partitions functions allowed by modularity constraints is finite dimensional. Given the above statement, this follows from the results of [37] on the finite dimensionality of spaces of vector valued modular forms.

6.2. A second proof of modularity. The goal of this section is to give a direct proof of the main modularity statement. The standard notation $e(x)=e^{2 \pi i x}$ will be used for brevity. To prove modularity, one needs to show that

$$
Z_{r, \delta}\left(-\frac{1}{\tau}\right)=\frac{\tau^{-1-\ell / 2} e\left(\frac{\ell-2}{8}\right)}{\sqrt{m} r^{\ell / 2}} \sum_{\delta^{\prime} \in G_{r}} e\left(-\left(\delta, \delta^{\prime}\right)_{r}\right) Z_{r, \delta^{\prime}}(\tau) .
$$

Recall that $Z_{r, \delta}(\tau)$ is given by

$$
Z_{r, \delta}(\tau)=\frac{1}{2 r^{2}} \sum_{\substack{k, l \in \mathbb{Z}_{\geq 1}, k l=r \\ \delta=f_{l, k}(\eta)}} \sum_{s=0}^{l-1} l e\left(-s \theta_{l}(\eta)\right) \Psi_{\eta_{1}}\left(\frac{k \tau+s}{l}\right) .
$$

where for any $k, l \in \mathbb{Z}, k, l \geq 1$

$$
f_{l, k}: \Lambda^{\vee} / l \Lambda \longrightarrow \Lambda^{\vee} /(k l) \Lambda
$$

is the injective morphism of lattices defined by $f_{l, k}\left([d]_{l}\right)=[k d]_{k l}$. In addition the surjective morphism of lattices

$$
g_{k, l}: \Lambda^{\vee} /(k l) \Lambda \longrightarrow \Lambda^{\vee} / l \Lambda, \quad g_{k, l}\left([d]_{k l}\right)=[d]_{l}
$$

will be also used in the proof. For any $\delta \in \Lambda^{\vee} /(k l) \Lambda$ let $\delta_{l}=g_{k, l}(\delta)$.

On needs to distinguish between the $s=0$ and $s>0$ cases. For $s>0$, let $p=\operatorname{gcd}(s, l)$ and $\tilde{s}=s / p, \tilde{l}=l / p$. Note that $0<\tilde{s}<\tilde{l}$. Then (6.7) can be rewritten as

$$
\begin{aligned}
Z_{r, \delta}(\tau)= & \frac{1}{2 r^{2}} \sum_{\substack{k, l \in \mathbb{Z}_{\geq 1}, k l=r \\
\delta=f_{l, k}(\eta)}} l \Psi_{\eta_{1}}\left(\frac{k \tau}{l}\right) \\
& +\frac{1}{2 r^{2}} \sum_{\substack{k, \tilde{l}, p \in \mathbb{Z}_{\geq 1}, k \tilde{l} p=r \\
\delta=f_{p \tilde{l}, k}(\eta)}} p \tilde{l} \sum_{\substack{1 \leq \tilde{s} \leq \tilde{l}-1 \\
(\tilde{s}, \tilde{l})=1}} e\left(-\tilde{s} \theta_{\tilde{l}}\left(\eta_{\tilde{l}}\right)\right) \Psi_{\eta_{1}}\left(\frac{k \tau+p \tilde{s}}{p \tilde{l}}\right) .
\end{aligned}
$$

Using the above formula, one can calculate $Z_{r, \delta}(-1 / \tau)$ as follows. For the $s=0$ terms note that

$$
\Psi_{\eta_{1}}\left(-\frac{k}{l \tau}\right)=\frac{l^{-1-\ell / 2} k^{1+\ell / 2} \tau^{-1-\ell / 2} e\left(\frac{\ell-2}{8}\right)}{\sqrt{m}} \sum_{\mu \in G_{1}} e\left(-\left(\eta_{1}, \mu\right)_{1}\right) \Psi_{\mu}\left(\frac{l \tau}{k}\right) .
$$

since $\Psi(\tau)$ is a vector valued modular form of weight $(-1-\ell / 2)$ and type $\rho_{\Lambda}$. In order to evaluate the $s \geq 1$ terms, one has to compute

$$
\Psi_{\eta_{1}}\left(-\frac{k}{p \tilde{l} \tau}+\frac{\tilde{s}}{\tilde{l}}\right)
$$


using the modular properties of $\Psi$. Let

$$
\tau^{\prime}=\frac{p}{k \tilde{l}} \tau+\frac{s^{\prime}}{\tilde{l}}
$$

where $s^{\prime} \in\{0, \ldots, l-1\}$ is uniquely defined by the requirement that $s^{\prime} \tilde{s}=-1 \bmod \tilde{l}$. Then, it is easy to check that

$$
-\frac{k}{p \tilde{l} \tau}+\frac{\tilde{s}}{\tilde{l}}=\frac{a \tau^{\prime}+b}{c \tau^{\prime}+d}
$$

for the $S L(2, \mathbb{Z})$ transformation

$$
\left(\begin{array}{ll}
a & b \\
c & d
\end{array}\right)=\left(\begin{array}{cc}
\tilde{s} & -\frac{\tilde{s} s^{\prime}+1}{\tilde{l}} \\
\tilde{l} & -s^{\prime}
\end{array}\right)
$$

Now note that Shintani's formula [57], reviewed in Appendix B, yields an identity:

$$
\Psi_{\eta_{1}}\left(-\frac{k}{p \tilde{l} \tau}+\frac{\tilde{s}}{\tilde{l}}\right)=\frac{p^{-1-\ell / 2} \tau^{-1-\ell / 2} k^{1+\ell / 2} \tilde{l}^{-\ell / 2} e\left(\frac{\ell-2}{8}\right)}{\sqrt{m}} \sum_{\sigma \in G_{1}} \bar{h}_{\tilde{l},-s^{\prime}, \tilde{s}}\left(\eta_{1}, \sigma\right) \Psi_{\sigma}\left(\frac{p}{k \tilde{l}} \tau+\frac{s^{\prime}}{\tilde{l}}\right)
$$

where $\bar{h}_{\tilde{l},-s^{\prime}, \tilde{s}}: G_{1} \times G_{1} \rightarrow \mathbb{C}$ is a function defined as follows. Pick any lift $\mu \in G_{\tilde{l}}$ of $\sigma$, such that $\mu_{1}=\sigma$. Then

$$
\bar{h}_{\tilde{l},-s^{\prime}, \tilde{s}}\left(\eta_{1}, \sigma\right)=\sum_{u \in \Lambda / \tilde{l} \Lambda} e\left(-\frac{s^{\prime}}{2}(\mu+u, \mu+u)_{\tilde{l}}-\left(\mu+u, \eta_{\tilde{l}}\right)_{\tilde{l}}+\frac{\tilde{s}}{2}\left(\eta_{\tilde{l}}, \eta_{\tilde{l}}\right)_{\tilde{l}}\right) .
$$

As shown in Appendix $\mathrm{B}$, the right hand side of the above equation does not depend on the chosen lift $\mu$.

Combining (6.9) and (6.13), one gets

$$
Z_{r, \delta}\left(-\frac{1}{\tau}\right)=\frac{\tau^{-1-\ell / 2} e\left(\frac{\ell-2}{8}\right)}{2 r^{2} \sqrt{m}}\left(S_{0}(\tau)+S_{\geq 1}(\tau)\right)
$$

with

$$
S_{0}(\tau)=\sum_{\substack{k, l \in \mathbb{Z}_{\geq 1}, k l=r \\ \delta=f_{l, k}(\eta)}} l^{-\ell / 2} k^{1+\ell / 2} \sum_{\sigma \in G_{1}} e\left(-\left(\eta_{1}, \sigma\right)_{1}\right) \Psi_{\sigma}\left(\frac{l \tau}{k}\right)
$$

and

$$
S_{\geq 1}(\tau)=\sum_{\substack{k, \tilde{l}, p \in \mathbb{Z}_{\geq 1}, k \tilde{l} p=r \\ \delta=f_{p \tilde{l}, k}(\eta)}} p^{-\ell / 2} \tilde{l}^{1-\ell / 2} k^{1+\ell / 2} \sum_{\substack{1 \leq \tilde{s} \leq \tilde{l}-1 \\(\tilde{s}, \tilde{l})=1}} e\left(-\tilde{s} \theta_{\tilde{l}}\left(\eta_{\tilde{l}}\right)\right) \sum_{\sigma \in G_{1}} \bar{h}_{\tilde{l},-s^{\prime}, \tilde{s}}\left(\eta_{1}, \sigma\right) \Psi_{\sigma}\left(\frac{p}{k \tilde{l}} \tau+\frac{s^{\prime}}{\tilde{l}}\right)
$$

Now note that

$$
e\left(-\tilde{s} \theta_{\tilde{l}}\left(\eta_{\tilde{l}}\right)\right) \bar{h}_{\tilde{l},-s^{\prime}, \tilde{s}}\left(\eta_{1}, \sigma\right)=\sum_{u \in \Lambda / \tilde{l} \Lambda} e\left(-\frac{s^{\prime}}{2}(\mu+u, \mu+u)_{\tilde{l}}-\left(\mu+u, \eta_{\tilde{l}}\right)_{\tilde{l}}\right)
$$


where $\mu \in G_{\tilde{l}}$ is an arbitrary lift of $\sigma \in G_{1}$. Again, as observed in Appendix B, the function $h_{\tilde{l},-s^{\prime}, \eta_{\tilde{l}}}: G_{\tilde{l}} \rightarrow \mathbb{C}$,

$$
h_{\tilde{l},-s^{\prime}, \eta_{\tilde{l}}}(\mu)=\sum_{u \in \Lambda / \tilde{l} \Lambda} e\left(-\frac{s^{\prime}}{2}(\mu+u, \mu+u)_{\tilde{l}}-\left(\mu+u, \eta_{\tilde{l}}\right)_{\tilde{l}}\right)
$$

is invariant under shifts $\mu \mapsto \mu+x, x \in \Lambda / \tilde{l} \Lambda$. Hence it descends to a function $\bar{h}_{\tilde{l},-s^{\prime}, \eta_{\tilde{l}}}: G_{1} \rightarrow \mathbb{C}$. Therefore

$$
S_{\geq 1}(\tau)=\sum_{\substack{k, \tilde{l}, p \in \mathbb{Z} \geq 1, k \tilde{l} p=r \\ \delta=f_{p \tilde{l}, k}(\eta)}} p^{-\ell / 2 \tilde{l}^{1-\ell / 2}} k^{1+\ell / 2} \sum_{\substack{1 \leq \tilde{s} \leq \tilde{l}-1 \\(\tilde{s}, \tilde{l})=1}} \sum_{\sigma \in G_{1}} \bar{h}_{\tilde{l},-s^{\prime}, \eta_{\tilde{l}}}(\sigma) \Psi_{\sigma}\left(\frac{p}{k \tilde{l}} \tau+\frac{s^{\prime}}{\tilde{l}}\right) .
$$

Returning to equation (6.6), one now has to prove that

$$
\frac{1}{r^{\ell / 2}} \sum_{\delta^{\prime} \in G_{r}} e\left(-\left(\delta, \delta^{\prime}\right)_{r}\right) Z_{r, \delta^{\prime}}(\tau)=\frac{1}{2 r^{2}}\left(S_{0}(\tau)+S_{\geq 1}(\tau)\right) .
$$

First, using (6.8) the left-hand-side can be written as

$$
\begin{aligned}
\frac{1}{r^{\ell / 2}} \sum_{\delta^{\prime} \in G_{r}} e\left(-\left(\delta, \delta^{\prime}\right)_{r}\right) Z_{r, \delta^{\prime}}(\tau)= & \frac{1}{2 r^{2} r^{\ell / 2}} \sum_{\delta^{\prime} \in G_{r}} e\left(-\left(\delta, \delta^{\prime}\right)_{r}\right)\left(\sum_{\substack{k, l \in \mathbb{Z}_{\geq 1}, k l=r \\
\delta^{\prime}=f_{l, k}(\eta)}} l \Psi_{\eta_{1}}\left(\frac{k \tau}{l}\right)\right. \\
& \left.+\sum_{\substack{k, \tilde{l}, p \in \mathbb{Z}_{\geq 1}, k \tilde{l} p=r \\
\delta^{\prime}=f_{p \tilde{l}, k}(\eta)}} p \tilde{l} \sum_{\substack{1 \leq \tilde{s} \leq \tilde{l}-1 \\
(\tilde{s}, \tilde{l})=1}} e\left(-\tilde{s} \theta_{\tilde{l}}\left(\eta_{\tilde{l}}\right)\right) \Psi_{\eta_{1}}\left(\frac{k \tau+p \tilde{s}}{p \tilde{l}}\right)\right) \\
= & \frac{1}{2 r^{2}}\left(Q_{0}(\tau)+Q_{\geq 1}(\tau)\right),
\end{aligned}
$$

where

$$
Q_{0}(\tau)=\frac{1}{r^{\ell / 2}} \sum_{\substack{k, l \in \mathbb{Z}_{\geq 1}, k l=r \\ \eta \in G_{l}}} l e\left(-\left(\delta_{l}, \eta\right)_{l}\right) \Psi_{\eta_{1}}\left(\frac{k \tau}{l}\right)
$$

and

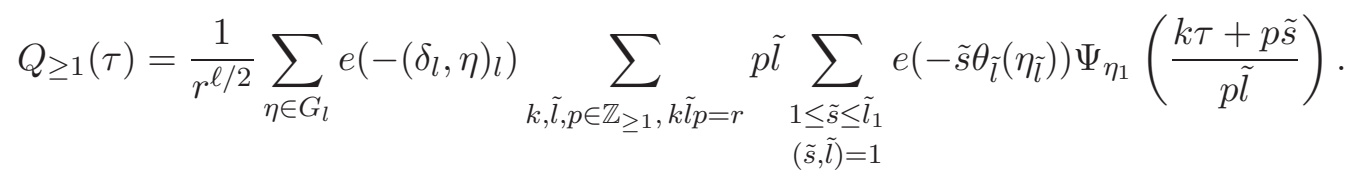

It will be shown below that $Q_{0}(\tau)=S_{0}(\tau)$ and $Q_{\geq 1}(\tau)=S_{\geq 1}(\tau)$.

In order to compute $Q_{0}(\tau)$ note that there is an exact sequence of finite abelian groups

$$
0 \rightarrow \Lambda / l \Lambda \rightarrow G_{l} \rightarrow G_{1} \rightarrow 0
$$

Given any element $\sigma \in G_{1}$, let $\mu \in G_{l}$ denote an arbitrary lift of $\sigma$. Then one has

$$
\sum_{\substack{\eta \in G_{l} \\ \eta_{1}=\sigma}} e\left(-\left(\delta_{l}, \eta\right)_{l}\right)=\sum_{u \in \Lambda / l \Lambda} e\left(-\left(\delta_{l}, \mu+u\right)_{l}\right)=e\left(-\left(\delta_{l}, \mu\right)_{l}\right) \sum_{u \in \Lambda / l \Lambda} e\left(-\left(\delta_{l}, u\right)_{l}\right) .
$$


Now note that

$$
\sum_{u \in \Lambda / l \Lambda} e\left(-\left(\delta_{l}, u\right)_{l}\right)= \begin{cases}l^{\ell} & \text { if } \delta=f_{k, l}(\xi) \text { for some } \xi \in G_{k} \\ 0 & \text { otherwise. }\end{cases}
$$

Moreover if $\delta=f_{k, l}(\xi)$ for some $\xi \in G_{k}$, then $e\left(-\left(\delta_{l}, \mu\right)_{l}\right)=e\left(-\left(\xi_{1}, \mu_{1}\right)_{1}\right)=e\left(-\left(\xi_{1}, \sigma\right)_{1}\right)$. Therefore,

$$
Q_{0}(\tau)=\sum_{\substack{k, l \in \mathbb{Z}_{\geq 1}, k l=r \\ \delta=f_{k, l}(\xi)}} k^{-\ell / 2} l^{1+\ell / 2} \sum_{\sigma \in G_{1}} e\left(-\left(\xi_{1}, \sigma\right)_{1}\right) \Psi_{\sigma}\left(\frac{k \tau}{l}\right) .
$$

Comparing with $S_{0}(\tau)$ in (6.15), exchanging the summation variables $l \leftrightarrow k$ and the symbols $\xi \leftrightarrow \eta$ yields

$$
Q_{0}(\tau)=S_{0}(\tau)
$$

as claimed above.

The computation of $Q_{\geq 1}(\tau)$ is similar. Recall that

$$
Q_{\geq 1}(\tau)=\frac{1}{r^{\ell / 2}} \sum_{\eta \in G_{l}} e\left(-\left(\delta_{l}, \eta\right)_{l}\right) \sum_{\substack { k, \tilde{l}, p \in \mathbb{Z}_{\geq 1}, k \tilde{l} p=r \\
\begin{subarray}{c}{1 \leq \tilde{s} \leq \tilde{l}-1 \\
(\tilde{s}, \tilde{l})=1{ k , \tilde { l } , p \in \mathbb { Z } _ { \geq 1 } , k \tilde { l } p = r \\
\begin{subarray} { c } { 1 \leq \tilde { s } \leq \tilde { l } - 1 \\
( \tilde { s } , \tilde { l } ) = 1 } }\end{subarray}} e\left(-\tilde{s} \theta_{\tilde{l}}\left(\eta_{\tilde{l}}\right)\right) \Psi_{\eta_{1}}\left(\frac{k \tau+p \tilde{s}}{p \tilde{l}}\right) .
$$

The right hand side of equation (6.24) can be written as

$$
\frac{1}{r^{\ell / 2}} \sum_{k, \tilde{l}, p \in \mathbb{Z}_{\geq 1}, k \tilde{l} p=r} p \tilde{l} \sum_{\eta \in G_{l}} e\left(-\left(\delta_{l}, \eta\right)_{l}\right) A\left(\eta_{\tilde{l}}\right)
$$

where

$$
A\left(\eta_{\tilde{l}}\right)=\sum_{\substack{1 \leq \tilde{s} \leq \tilde{l}-1 \\(\tilde{s}, \tilde{l})=1}} e\left(-\tilde{s} \theta_{\tilde{l}}\left(\eta_{\tilde{l}}\right)\right) \Psi_{\eta_{1}}\left(\frac{k \tau+p \tilde{s}}{p \tilde{l}}\right)
$$

depends only on $\tilde{l}, \eta_{\tilde{l}}$. In order to compute the sum over $\eta \in G_{l}$ with fixed projection $\gamma=\eta_{\tilde{l}} \in G_{\tilde{l}}$, note that there is an exact sequence of finite abelian groups

$$
0 \rightarrow \Lambda / p \Lambda \stackrel{i}{\rightarrow} G_{l} \rightarrow G_{\tilde{l}} \rightarrow 0 .
$$

Given an element $v \in \Lambda / p \Lambda$, one has $i(v)=[\tilde{l} \alpha]_{l}$ where $\alpha \in \Lambda$ is an arbitrary representative of $v$. The right hand side, $[\tilde{l} \alpha]_{l} \in G_{l}$ is clearly independent of the choice of $\alpha$. Then let $\gamma_{0} \in G_{l}$ denote an arbitrary lift of $\gamma$ and note that

$$
\sum_{\substack{\eta \in G_{l} \\ \eta_{\bar{l}}=\gamma}} e\left(-\left(\delta_{l}, \eta\right)_{l}\right)=e\left(-\left(\delta_{l}, \gamma_{0}\right)_{l}\right) \sum_{v \in \Lambda / p \Lambda} e\left(-\left(\delta_{p}, v\right)_{p}\right)
$$

since $l=p \tilde{l}$. As before,

$$
\sum_{v \in \Lambda / p \Lambda} e\left(-\left(\delta_{p}, v\right)_{p}\right)= \begin{cases}p^{\ell} & \text { if } \delta=f_{k \tilde{l}, p}(\xi) \text { for some } \xi \in G_{k \tilde{l}} \\ 0 & \text { otherwise. }\end{cases}
$$


Moreover, if $\delta=f_{k \tilde{l}, p}(\xi)$ then

$$
e\left(-\left(\delta_{l}, \gamma_{0}\right)_{l}\right)=e\left(-\left(\xi_{\tilde{l}}, \gamma\right)_{\tilde{l}}\right)
$$

Therefore,

$$
Q_{\geq 1}(\tau)=\sum_{\substack{k, \tilde{l}, p \in \mathbb{Z}_{\geq 1}, k \tilde{k} p=r \\ \delta=f_{k \tilde{l}, p}(\xi)}} k^{-\ell / 2} p^{1+\ell / 2} \tilde{l}^{1-\ell / 2} \sum_{\substack{1 \leq \tilde{s} \leq \tilde{l}-1 \\(\tilde{s}, \tilde{l})=1}} \sum_{\gamma \in G_{\tilde{l}}} e\left(-\left(\xi_{\tilde{l}}, \gamma\right)_{\tilde{l}}-\tilde{s} \theta_{\tilde{l}}(\gamma)\right) \Psi_{\gamma_{1}}\left(\frac{k \tau+p \tilde{s}}{p \tilde{l}}\right)
$$

Finally, using again the exact sequence

$$
0 \rightarrow \Lambda / \tilde{l} \Lambda \rightarrow G_{\tilde{l}} \rightarrow G_{1} \rightarrow 0
$$

note that

$$
\sum_{\substack{\gamma \in G_{\tilde{l}} \\ \gamma_{1}=\rho}} e\left(-\left(\xi_{\tilde{l}}, \gamma\right)_{\tilde{l}}-\tilde{s} \theta_{\tilde{l}}(\gamma)\right)=\sum_{v \in \Lambda / \tilde{l} \Lambda} e\left(-\frac{\tilde{s}}{2}\left(\rho_{0}+v, \rho_{0}+v\right)_{\tilde{l}}-\left(\rho_{0}+v, \xi_{\tilde{l}}\right)_{\tilde{l}}\right)
$$

where $\rho_{0} \in G_{\tilde{l}}$ is an arbitrary lift of $\rho \in G_{1}$. Therefore

$$
\sum_{\substack{\gamma \in G_{\tilde{I}} \\ \gamma_{1}=\rho}} e\left(-\left(\xi_{\tilde{l}}, \gamma\right)_{\tilde{l}}-\tilde{s} \theta_{\tilde{l}}(\gamma)\right)=h_{\tilde{l},-\tilde{s}, \xi_{\tilde{l}}}\left(\rho_{0}\right)
$$

where $h_{\tilde{l},-\tilde{s}, \xi_{\tilde{l}}}: G_{\tilde{l}} \rightarrow \mathbb{C}$ is the function defined in (6.16), which descends to the function $\bar{h}_{\tilde{l},-\tilde{s}, \xi_{\tilde{l}}}$ : $G_{1} \rightarrow \mathbb{C}$. Hence one obtains

$$
\sum_{\substack{\gamma \in G_{\tilde{\tau}} \\ \gamma 1=\rho}} e\left(-\left(\xi_{\tilde{l}}, \gamma\right)_{\tilde{l}}-\tilde{s} \theta_{\tilde{l}}(\gamma)\right)=\bar{h}_{\tilde{l},-\tilde{s}, \xi_{\tilde{l}}}(\rho)
$$

independent of the choice of $\rho_{0}$. In conclusion the final expression for $Q_{\geq 1}(\tau)$ is

$$
Q_{\geq 1}(\tau)=\sum_{\substack{k, \tilde{l}, p \in \mathbb{Z}_{\geq 1}, k \tilde{l} p=r \\ \delta=f_{k \tilde{l}, p}(\xi)}} k^{-\ell / 2} p^{1+\ell / 2} \tilde{l}^{1-\ell / 2} \sum_{\substack{1 \leq \tilde{s} \leq \tilde{l}-1 \\(\tilde{s}, \tilde{l})=1}} \sum_{\rho \in G_{1}} \bar{h}_{\tilde{l},-\tilde{s}, \xi_{\tilde{l}}}(\rho) \Psi_{\rho}\left(\frac{k \tau+p \tilde{s}}{p \tilde{l}}\right)
$$

Comparing with (6.17), note that exchanging the summation variables $p \leftrightarrow k, s^{\prime} \leftrightarrow \tilde{s}$, and the symbols $\xi \leftrightarrow \eta$ yields

$$
Q_{\geq 1}(\tau)=S_{\geq 1}(\tau)
$$

concluding the proof. 


\section{Appendix A. Weil Representations and sublattices of the K3 LAtTice}

This section contains some basic facts on Weil representations with applications to sublattices of the middle cohomology lattice $H^{2}(S, \mathbb{Z})$ of a smooth generic K3 surface $S$.

First recall the definition of the Weil representation associated to any lattice $\Upsilon$ equipped with an integral even nondegenerate symmetric bilinear form $(,)_{\Upsilon}$ of signature $\left(b_{+}, b_{-}\right)$. Let $\kappa \in \mathbb{Z}$ denote the absolute value of the determinant of the bilinear form and let $\Upsilon^{\vee}=\operatorname{Hom}(\Upsilon, \mathbb{Z})$ be the dual lattice. For brevity let $\Upsilon_{\mathbb{Q}}=\Upsilon \otimes_{\mathbb{Z}} \mathbb{Q}$. Moreover the natural extension of $(,)_{\Upsilon}$ to $\Upsilon_{\mathbb{Q}}$ by $\mathbb{Q}$-linearity will be also denoted by $(,)_{\Upsilon}$. The distinction will be clear from the context.

The given bilinear form determines a lattice embedding $\Upsilon \hookrightarrow \Upsilon^{\vee}$ such that $Q=\Upsilon^{\vee} / \Upsilon$ is a finite abelian group of rank $\kappa$. Note that the bilinear form $(,) \Upsilon$ extends by $\mathbb{Q}$-linearity to $\Upsilon^{\vee} \subset \Upsilon_{\mathbb{Q}}$, hence it descends to a $\mathbb{Q} / \mathbb{Z}$-valued bilinear pairing $(,)_{Q}$ on $Q$. For any two equivalence classes $\delta_{1}, \delta_{2} \in Q$ one has

$$
\left(\delta_{1}, \delta_{2}\right)_{Q}=\left(\xi_{1}, \xi_{2}\right) \Upsilon \bmod \mathbb{Z}
$$

where $\xi_{1}, \xi_{2} \in \Upsilon^{\vee}$ are arbitrary representatives of $\delta_{1}, \delta_{2}$ respectively.

According to [67] the pair $\Upsilon$, ( , ) $\Upsilon$ determines a unitary symmetric representation of the metaplectic group $M p(2, \mathbb{Z})$. For completeness, recall that in order to define the metaplectic group one has to pick a complex number $\tau$ with $\operatorname{Im}(\tau)>0$. Then $M p(2, \mathbb{Z})$ is the double cover of $S L(2, \mathbb{Z})$ consisting of pairs

$$
(\sigma, \sqrt{c \tau+d}), \quad \sigma=\left(\begin{array}{ll}
a & b \\
c & d
\end{array}\right) \in S L(2, \mathbb{Z}) .
$$

This group is generated by

$$
\widetilde{T}=(T, 1), \quad \widetilde{S}=(S, \sqrt{\tau}),
$$

satisfying the relations $(\widetilde{S})^{2}=(\widetilde{S} \widetilde{T})^{3}=\left(-I_{2}, i\right)$, which are independent of the choice of $\tau$.

Let $\mathbb{C}\left[\Upsilon^{\vee} / \Upsilon\right]$ be the complex vector space generated by the basis elements $e_{\delta}$ with $\delta \in Q$. Then the pair $\Upsilon,(,) \Upsilon$ determines a representation $\rho_{\Upsilon}: M p(2, \mathbb{Z}) \rightarrow \operatorname{End}\left(\mathbb{C}\left[\Upsilon^{\vee} / \Upsilon\right]\right)$ given by

$$
\rho_{\Upsilon}(\widetilde{T})\left(e_{\delta}\right)=e^{2 \pi i(\delta, \delta)_{Q} / 2} e_{\delta}, \quad \rho_{\Lambda}(\widetilde{S})\left(e_{\delta}\right)=\frac{e^{\pi i\left(b_{-}-b_{+}\right) / 4}}{\sqrt{\kappa}} \sum_{\delta^{\prime} \in Q} e^{-2 \pi i\left(\delta, \delta^{\prime}\right) Q_{Q}} e_{\delta^{\prime}}
$$

This representation is unitary symmetric, which implies that the dual representation $\rho_{\Upsilon}^{*}$ is isomorphic to its complex conjugate.

Now let $\Lambda_{K 3}$ be the middle cohomology lattice of a smooth generic K3 surface. This lattice has rank 22 and is equipped with the natural intersection form $(,)_{K 3}$ which is an integral even nondegenerate symmetric bilinear form of determinant 1. Let $\Lambda \subset \Lambda_{K 3}$ be a rank $1 \leq \ell \leq 20$ sublattice such that the intersection form restricts to a nondegenerate symmetric bilinear form $(,)_{\Lambda}$ on $\Lambda$ of signature $(1, \ell-1)$. Let $\Lambda^{\perp} \subset \Lambda_{K 3}$ denote the sublattice consisting of all elements $u \in \Lambda_{K 3}, u \cdot \Lambda=0$, and $(,)_{\Lambda^{\perp}}$ denote the induced nondegenerate symmetric bilinear form on $\Lambda^{\perp}$. 
The later has signature $(2,20-\ell)$. Then the lattices $\Lambda, \Lambda^{\perp}$ equipped with the induced bilinear forms determine the Weil representations $\rho_{\Lambda}, \rho_{\Lambda^{\perp}}$. Below it will be shown that there is an isomorphism

$$
\rho_{\Lambda^{\perp}}^{*} \simeq \rho_{\Lambda}
$$

of representations of the metaplectic group.

Let $G=\Lambda^{\vee} / \Lambda, G_{\perp}=\left(\Lambda^{\perp}\right)^{\vee} / \Lambda^{\perp}$. It suffices to prove that there is an isomorphism of finite abelian groups $f: G_{\perp} \stackrel{\sim}{\longrightarrow} G$ such that

$$
\left(\xi_{1}, \xi_{2}\right)_{G_{\perp}}+\left(f\left(\xi_{1}\right), f\left(\xi_{2}\right)\right)_{G}=0
$$

in $\mathbb{Q} / \mathbb{Z}$ for any $\xi_{1}, \xi_{2} \in G_{\perp}$. The starting point is the observation that the natural projections

$$
p:\left(\Lambda_{K 3}\right)_{\mathbb{Q}} \rightarrow \Lambda_{\mathbb{Q}} \quad p_{\perp}:\left(\Lambda_{K 3}\right)_{\mathbb{Q}} \rightarrow \Lambda_{\mathbb{Q}}^{\perp}
$$

with respect to the intersection form determine lattice isomorphisms

$$
\phi: \Lambda_{K 3} / \Lambda^{\perp} \stackrel{\sim}{\longrightarrow} \Lambda^{\vee}, \quad \phi_{\perp}: \Lambda_{K 3} / \Lambda \stackrel{\sim}{\longrightarrow}\left(\Lambda^{\perp}\right)^{\vee} .
$$

This follows easily since the intersection form on $\Lambda_{K 3}$ is unimodular. Then one further obtains isomorphisms of finite abelian groups

$$
\bar{\phi}: \Lambda_{K 3} /\left(\Lambda \oplus \Lambda^{\perp}\right) \rightarrow G, \quad \bar{\psi}: \Lambda_{K 3} /\left(\Lambda \oplus \Lambda^{\perp}\right) \rightarrow G_{\perp} .
$$

In particular there is an isomorphism $f=\bar{\phi} \circ \bar{\psi}^{-1}: G_{\perp} \rightarrow G$.

Next let $u_{1}, u_{2} \in \Lambda_{K 3}$ be arbitrary elements. Note that

$$
\left(p\left(u_{i}\right), p\left(u_{i}\right)\right)_{\Lambda}=\left(p\left(u_{i}\right), p\left(u_{i}\right)\right)_{K 3}, \quad\left(p_{\perp}\left(u_{i}\right), p_{\perp}\left(u_{i}\right)\right)_{\Lambda^{\perp}}=\left(p_{\perp}\left(u_{i}\right), p_{\perp}\left(u_{i}\right)\right)_{K 3}
$$

for $i \in\{1,2\}$. Since

$$
\left(\Lambda_{K 3}\right)_{\mathbb{Q}}=\Lambda_{\mathbb{Q}} \oplus \Lambda_{\mathbb{Q}}^{\perp} .
$$

one has $u_{i}=p\left(u_{i}\right)+p_{\perp}\left(u_{i}\right)$, for $i \in\{1,2\}$, which yields

$$
\left(p\left(u_{1}\right), p\left(u_{2}\right)\right)_{\Lambda}+\left(p_{\perp}\left(u_{1}\right), p_{\perp}\left(u_{2}\right)\right)_{\Lambda^{\perp}}=\left(u_{1}, u_{2}\right)_{K 3} \in \mathbb{Z} .
$$

This implies relation (A.3).

\section{Appendix B. Shintani's Formula}

This section is a brief review of Shintani's explicit formula [57, Prop. 1.6] for the matrix elements of the Weil representation. As in Section 6, consider a lattice $\Lambda$ equipped with an integral even nondegenerate symmetric bilinear form. Let $\ell$ denote the rank of $\Lambda$ and $m$ denote the absolute value of the determinant of the given bilinear form. As explained in the previous section, such a lattice determines a representation

$$
\rho_{\Lambda}: M p(2, \mathbb{Z}) \rightarrow \operatorname{End}\left(\mathbb{C}\left[G_{1}\right]\right)
$$


where $M p(2, \mathbb{Z})$ is the metaplectic double cover of $S L(2, \mathbb{Z})$ and $G_{1}=\Lambda^{\vee} / \Lambda$. The action of the generators of the metaplectic group is given in (A.1). Shintani's result [57, Prop. 1.6] provides an explicit formula for the matrix elements associated to an arbitrary element

$$
\tilde{\sigma}=(\sigma, \sqrt{c \tau+d}), \quad \sigma=\left(\begin{array}{cc}
a & b \\
c & d
\end{array}\right)
$$

of the metaplectic group with $c>0$. In order to write this formula in detail consider first the function

$$
\begin{gathered}
f_{c, a, d}: \Lambda^{\vee} / c \Lambda \times \Lambda^{\vee} / c \Lambda \times \Lambda / c \Lambda \rightarrow \mathbb{C} \\
f_{c, a, d}(\gamma, \zeta, u)=e\left(\frac{a}{2}(\gamma+u, \gamma+u)_{c}-(\gamma+u, \zeta)_{c}+\frac{d}{2}(\zeta, \zeta)_{c}\right)
\end{gathered}
$$

Next define a function

$$
\begin{gathered}
g_{c, a, d}: \Lambda^{\vee} / c \Lambda \times \Lambda^{\vee} / c \Lambda \rightarrow \mathbb{C} \\
g_{c, a, d}(\gamma, \zeta)=\sum_{u \in \Lambda / c \Lambda} f_{c, a, d}(\gamma, \zeta, u) .
\end{gathered}
$$

Then one checks as follows that the function $g_{a, c, d}$ satisfies the invariance conditions

$$
g_{c, a, d}(\gamma+x, \zeta)=g_{c, a, d}(\gamma, \zeta+y)=g_{c, a, d}(\gamma, \zeta)
$$

for any $(\gamma, \zeta) \in \Lambda^{\vee} / c \Lambda \times \Lambda^{\vee} / c \Lambda$ and any $(x, y) \in \Lambda / c \Lambda \times \Lambda / c \Lambda$.

Invariance under $\gamma \mapsto \gamma+x$ is easily proven by changing the summation variable $u$ to $u-x$ in the right hand side of $(\underline{B .3})$.

Invariance under $\zeta \mapsto \zeta+y$ requires more work. Recall that the pairing $(,)_{c}$ takes integral values on $\Lambda^{\vee} / c \Lambda \times \Lambda / c \Lambda$ and restricts to an even integral form on $\Lambda / c \Lambda \times \Lambda / c \Lambda$. Then, using the basic relation $a d-b c=1$, one has the folllowing sequence of congruences $\bmod 2 \mathbb{Z}$ :

$$
\begin{aligned}
& a(\gamma+u, \gamma+u)_{c}-2(\gamma+u, \zeta+y)_{c}+d(\zeta+y, \zeta+y)_{c} \equiv \\
& a(\gamma+u, \gamma+u)_{c}-2 a d(\gamma+u, y)_{c}+a d^{2}(y, y)_{c}-2(\gamma+u-d y, \zeta)_{c}+d(\zeta, \zeta)_{c} \equiv \\
& a(\gamma+u-d y, \gamma+u-d y)_{c}-2(\gamma+u-d y, \zeta)_{c}+d(\zeta, \zeta)_{c} .
\end{aligned}
$$

Now invariance reduces again to a shift $u \mapsto u+d y$ of the summation variable in (B.3).

In conclusion $g_{a, c, d}$ descends to a function

$$
\bar{g}_{c, a, d}: \Lambda^{\vee} / \Lambda \times \Lambda^{\vee} / \Lambda \rightarrow \mathbb{C}
$$

Then Shintani's formula reads

$$
\rho_{\Lambda}(\tilde{\sigma})_{\delta_{1}, \delta_{2}}=\frac{e\left(\frac{\ell-2}{8}\right)}{c^{\ell / 2} m^{1 / 2}} \bar{g}_{c, a, d}\left(\delta_{1}, \delta_{2}\right) .
$$

A slightly different presentation of Shintani's formula will be needed for the modularity proof in Section ??. Namely let

$$
f_{c, a, d}^{\prime}: \Lambda^{\vee} / c \Lambda \times \Lambda^{\vee} / c \Lambda \times \Lambda / c \Lambda \rightarrow \mathbb{C}
$$


be the function defined by

$$
f_{c, a, d}^{\prime}(\gamma, \zeta, u)=e\left(\frac{a}{2}(\gamma, \gamma)_{c}-(\gamma, \zeta+u)_{c}+\frac{d}{2}(\zeta+u, \zeta+u)_{c}\right)
$$

Let

$$
h_{c, a, d}: \Lambda^{\vee} / c \Lambda \times \Lambda^{\vee} / c \Lambda \rightarrow \mathbb{C}
$$

be defined by

$$
h_{c, a, d}(\gamma, \zeta)=\sum_{u \in \Lambda / c \Lambda} f_{c, a, d}^{\prime}(\gamma, \zeta, u)
$$

Then by analogy with the above argument for $g_{c, a, d}$, the function $h_{c, a, d}$ also descends to a function

$$
\bar{h}_{c, a, d}: \Lambda^{\vee} / \Lambda \times \Lambda^{\vee} / \Lambda \rightarrow \mathbb{C} \text {. }
$$

Furthermore it will be shown below that

$$
h_{c, a, d}(\gamma, \zeta)=g_{c, a, d}(\gamma, \zeta)
$$

for any $(\gamma, \zeta)$.

By analogy with equation (B.2) one has $a(\gamma, \gamma)_{c}-2(\gamma, \zeta+u)_{c}+d(\zeta+u, \zeta+u)_{c} \equiv a(\gamma-d u, \gamma-d u)_{c}-2(\gamma-d u, \zeta)_{c}+d(\zeta, \zeta)_{c} \quad \bmod 2 \mathbb{Z}$.

Hence

$$
h_{c, a, d}(\gamma, \zeta)=\sum_{u \in \Lambda / c \Lambda} f_{c, a, d}(\gamma, \zeta,-d u)
$$

Now note that $c, d$ are coprime since $a d-b c=1$. Since $\Lambda / c \Lambda \simeq(\mathbb{Z} / c \mathbb{Z})^{\times \ell}$, this implies that multiplication by $d$ gives an isomorphism $\Lambda / c \Lambda \stackrel{d}{\longrightarrow} \Lambda / c \Lambda$. Therefore

$$
\sum_{u \in \Lambda / c \Lambda} f_{c, a, d}(\gamma, \zeta,-d u)=\sum_{u \in \Lambda / c \Lambda} f_{c, a, d}(\gamma, \zeta, u)
$$

which proves the claim.

Finally, note that a closely related function $\bar{h}_{c, d, \gamma}: \Lambda^{\vee} / \Lambda \rightarrow \mathbb{C}$ is also used in Section ?? . For any fixed element $\gamma \in \Lambda^{\vee} / c \Lambda$, define

$$
h_{c, d, \gamma}: \Lambda^{\vee} / c \Lambda \rightarrow \mathbb{C}
$$

by

$$
h_{c, d, \gamma}(\zeta)=\sum_{u \in \Lambda / c \Lambda} e\left(\frac{d}{2}(\zeta+u, \zeta+u)_{c}-(\zeta+u, \gamma)_{c}\right)
$$

Then it follows again that $h_{c, d, \gamma}(\zeta+y)=h_{c, d, \gamma}(\zeta)$ for any $\zeta \in \Lambda^{\vee} / c \Lambda, y \in \Lambda / c \Lambda$. Hence $h_{c, d, \gamma}$ descends to a function $\bar{h}_{c, d, \gamma}: \Lambda^{\vee} / \Lambda \rightarrow \mathbb{C}$. 


\section{REFERENCES}

[1] M. Aganagic, D. Jafferis, and N. Saulina. Branes, black holes and topological strings on toric Calabi-Yau manifolds. JHEP, 12:018, 2006.

[2] M. Aganagic, H. Ooguri, N. Saulina, and C. Vafa. Black holes, q-deformed 2d Yang-Mills, and non- perturbative topological strings. Nucl. Phys., B715:304-348, 2005.

[3] S. Alexandrov, J. Manschot, and B. Pioline. D3-instantons, Mock Theta Series and Twistors. JHEP, 04:002, 2013. arXiv:1207.1109.

[4] P. S. Aspinwall. K3 surfaces and string duality. In Fields, strings and duality. Proceedings, Summer School, Theoretical Advanced Study Institute in Elementary Particle Physics, TASI'96, Boulder, USA, June 2-28, 1996, pages 421-540, 1996.

[5] K. Behrend. Donaldson-Thomas type invariants via microlocal geometry. Ann. of Math. (2), 170(3):1307-1338, 2009.

[6] R. E. Borcherds. Automorphic forms with singularities on Grassmannians. Invent. Math., 132(3):491-562, 1998.

[7] R. E. Borcherds. The Gross-Kohnen-Zagier theorem in higher dimensions. Duke Math. J., 97(2):219-233, 1999.

[8] V. Bouchard, T. Creutzig, and D.-E. Diaconescu. Hecke operators for vector valued modular forms of the weil representation. work in progress.

[9] C. Brav, V. Bussi, D. Dupont, D. Joyce, and B. Szendrői. Symmetries and stabilization for sheaves of vanishing cycles. J. Singul., 11:85-151, 2015. With an appendix by Jörg Schürmann.

[10] K. Bringmann, T. Creutzig, and L. Rolen. Negative index Jacobi forms and quantum modular forms. Res. Math. Sci., 1:Art. 11, 32, 2014.

[11] J. H. Bruinier and O. Stein. The Weil representation and Hecke operators for vector valued modular forms. Math. Z., 264(2):249-270, 2010.

[12] A. Dabholkar, F. Denef, G. W. Moore, and B. Pioline. Exact and asymptotic degeneracies of small black holes. JHEP, 08:021, 2005.

[13] A. Dabholkar, F. Denef, G. W. Moore, and B. Pioline. Precision counting of small black holes. JHEP, 0510:096, 2005.

[14] A. Dabholkar and J. A. Harvey. Nonrenormalization of the Superstring Tension. Phys. Rev. Lett., 63:478, 1989.

[15] J. de Boer, M. C. N. Cheng, R. Dijkgraaf, J. Manschot, and E. Verlinde. A Farey Tail for Attractor Black Holes. JHEP, 11:024, 2006. hep-th/0608059.

[16] F. Denef and G. W. Moore. Split states, entropy enigmas, holes and halos. JHEP, 1111:129, 2011. hep-th/0702146.

[17] D.-E. Diaconescu. Vertical sheaves and Fourier-Mukai transform on elliptic Calabi-Yau threefolds. preprint, arXiv:1509.07749.

[18] D. E. Diaconescu and G. W. Moore. Crossing the Wall: Branes vs. Bundles. arXiv.org:hep-th/0706.3193.

[19] T. Dimofte and S. Gukov. Refined, Motivic, and Quantum. Lett. Math. Phys., 91:1, 2010.

[20] D. Gaiotto, A. Strominger, and X. Yin. The M5-brane elliptic genus: modularity and BPS states. J. High Energy Phys., (8):070, 18 pp. (electronic), 2007. hep-th/0607010.

[21] A. Gholampour, Y. Jianh, and M. Kool. Sheaves on weighted projective planes and modular forms. arXiv:1209.3922, 2012.

[22] A. Gholampour and A. Sheshmani. Donaldson-Thomas Invariants of 2-Dimensional sheaves inside threefolds and modular forms. arXiv:1309.0050.

[23] A. Gholampour and A. Sheshmani. Generalized Donaldson-Thomas Invariants of 2-Dimensional sheaves on local $\mathrm{P}^{\wedge}$ 2. Adv. Theor. Math. Physics. (To appear).

[24] A. Gholampour, A. Sheshmani, and Y. Toda. Stable pairs on nodal K3 fibrations. arXiv:1308.4722, 2013. 
[25] L. Göttsche. The Betti numbers of the Hilbert scheme of points on a smooth projective surface. Math. Ann., 286(1-3):193-207, 1990.

[26] L. Göttsche. Theta functions and Hodge numbers of moduli spaces of sheaves on rational surfaces. Comm. Math. Phys., 206(1):105-136, 1999.

[27] L. Göttsche, H. Nakajima, and K. Yoshioka. Donaldson = Seiberg-Witten from Mochizuki's formula and instanton counting. Publ. Res. Inst. Math. Sci., 47(1):307-359, 2011.

[28] J. A. Harvey and G. W. Moore. Algebras, BPS states, and strings. Nucl. Phys., B463:315-368, 1996. hepth/9510182.

[29] J. A. Harvey and G. W. Moore. On the algebras of BPS states. Commun. Math. Phys., 197:489-519, 1998. hep-th/9609017.

[30] D. Huybrechts and M. Lehn. The geometry of moduli spaces of sheaves. Aspects of Mathematics, E31. Friedr. Vieweg \& Sohn, Braunschweig, 1997.

[31] D. L. Jafferis and N. Saulina. Fragmenting D4 branes and coupled q-deformed Yang Mills. 2007.

[32] D. Joyce and Y. Song. A theory of generalized Donaldson-Thomas invariants. Mem. Amer. Math. Soc., 217(1020):iv+199, 2012.

[33] S. Katz. Genus zero Gopakumar-Vafa invariants of contractible curves. math.AG/0601193.

[34] S. H. Katz, A. Klemm, and C. Vafa. M theory, topological strings and spinning black holes. Adv. Theor.Math.Phys., 3:1445-1537, 1999.

[35] A. Klemm, J. Manschot, and T. Wotschke. Quantum geometry of elliptic Calabi-Yau manifolds. Commun. Number Theory Phys., 6(4):849-917, 2012.

[36] A. Klemm, D. Maulik, R. Pandharipande, and E. Scheidegger. Noether-Lefschetz theory and the Yau-Zaslow conjecture. J. Amer. Math. Soc., 23(4):1013-1040, 2010.

[37] M. Knopp and G. Mason. Vector-valued modular forms and Poincaré series. Illinois J. Math., 48(4):1345-1366, 2004.

[38] M. Kontsevich and Y. Soibelman. Stability structures, Donaldson-Thomas invariants and cluster transformations. arXiv.org:0811.2435.

[39] S. S. Kudla and J. J. Millson. Intersection numbers of cycles on locally symmetric spaces and Fourier coefficients of holomorphic modular forms in several complex variables. Inst. Hautes Études Sci. Publ. Math., (71):121-172, 1990.

[40] J. M. F. Labastida and C. Lozano. The Vafa-Witten theory for gauge group SU(N). Adv. Theor. Math. Phys., 3:1201-1225, 1999. hep-th/9903172.

[41] J. M. Maldacena, A. Strominger, and E. Witten. Black hole entropy in M theory. JHEP, 12:002, 1997.

[42] J. Manschot. The Betti numbers of the moduli space of stable sheaves of rank 3 on $\mathbb{P}^{2}$. Lett. Math. Phys., 98(1):65-78, 2011.

[43] J. Manschot. Wall-crossing of D4-branes using flow trees. Adv. Theor. Math. Phys., 15(1):1-42, 2011. arXiv:1003.1570.

[44] J. Manschot. BPS invariants of $N=4$ gauge theory on Hirzebruch surfaces. Commun. Number Theory Phys., 6(2):497-516, 2012.

[45] J. Manschot. BPS invariants of semi-stable sheaves on rational surfaces. Lett. Math. Phys., 103(8):895-918, 2013.

[46] D. Maulik and R. Pandharipande. Gromov-Witten theory and Noether-Lefschetz theory. In A celebration of algebraic geometry, volume 18 of Clay Math. Proc., pages 469-507. Amer. Math. Soc., Providence, RI, 2013.

[47] J. Milnor. Singular points of complex hypersurfaces. Annals of Mathematics Studies, No. 61. Princeton University Press, Princeton, N.J.; University of Tokyo Press, Tokyo, 1968. 
[48] J. A. Minahan, D. Nemeschansky, C. Vafa, and N. P. Warner. E-strings and $N=4$ topological Yang-Mills theories. Nuclear Phys. B, 527(3):581-623, 1998.

[49] S. Mukai. Symplectic structure of the moduli space of sheaves on an abelian or K3 surface. Invent. Math., 77(1):101-116, 1984.

[50] T. Nishinaka and S. Yamaguchi. Affine $S U(N)$ algebra from wall-crossings. JHEP, 07:030, 2014.

[51] T. Nishinaka, S. Yamaguchi, and Y. Yoshida. Two-dimensional crystal melting and D4-D2-D0 on toric CalabiYau singularities. JHEP, 05:139, 2014.

[52] T. Nishinaka and Y. Yoshida. A Note on statistical model for BPS D4-D2-D0 states. Phys. Lett., B711:132-138, 2012.

[53] H. Ooguri, A. Strominger, and C. Vafa. Black hole attractors and the topological string. Phys. Rev., D70:106007, 2004.

[54] R. Pandharipande and R. P. Thomas. The Katz-Klemm-Vafa conjecture for K3 surfaces.

[55] R. Pandharipande and R. P. Thomas. Curve counting via stable pairs in the derived category. Invent. Math., 178(2):407-447, 2009.

[56] N. R. Scheithauer. Some constructions of modular forms for the Weil representation of SL(2, Z). Nagoya Math. J., 220:1-43, 2015.

[57] T. Shintani. On construction of holomorphic cusp forms of half integral weight. Nagoya Math. J., 58:83-126, 1975.

[58] R. P. Thomas. A holomorphic Casson invariant for Calabi-Yau 3-folds, and bundles on K3 fibrations. J. Differential Geom., 54(2):367-438, 2000.

[59] Y. Toda. S-duality for surfaces with $a_{n}$-type singularities. arXiv:1312.2300, 2013.

[60] Y. Toda. Generalized donaldson-thomas invariants on the local projective plane. arXiv:1405.3366, 2014.

[61] Y. Toda. Flops and the S-duality conjecture. Duke Math. J., 164(12):2293-2339, 2015.

[62] C. Vafa. Gas of D-branes and Hagedorn density of BPS states. Nucl. Phys., B463:415-419, 1996.

[63] C. Vafa. Black holes and Calabi-Yau threefolds. Adv. Theor. Math. Phys., 2:207-218, 1998.

[64] C. Vafa. Two dimensional Yang-Mills, black holes and topological strings. 2004.

[65] C. Vafa and E. Witten. A Strong coupling test of S duality. Nucl. Phys., B431:3-77, 1994.

[66] M. Waldschmidt, P. Moussa, J. M. Luck, and C. Itzykson, editors. From number theory to physics. SpringerVerlag, Berlin, 1992. Papers from the Meeting on Number Theory and Physics held in Les Houches, March 7-16, 1989.

[67] A. Weil. Sur certains groupes d'opérateurs unitaires. Acta Math., 111:143-211, 1964.

[68] K. Yoshioka. The Betti numbers of the moduli space of stable sheaves of rank 2 on $\mathbf{P}^{2}$. J. Reine Angew. Math., 453:193-220, 1994.

[69] K. Yoshioka. The Betti numbers of the moduli space of stable sheaves of rank 2 on a ruled surface. Math. Ann., 302(3):519-540, 1995.

[70] K. Yoshioka. Betti numbers of moduli of stable sheaves on some surfaces. Nuclear Phys. B Proc. Suppl., 46:263268, 1996. S-duality and mirror symmetry (Trieste, 1995).

[71] K. Yoshioka. Euler characteristics of SU(2) instanton moduli spaces on rational elliptic surfaces. Comm. Math. Phys., 205(3):501-517, 1999.

1 Department of Mathematical and Statistical Sciences, University of Alberta, Edmonton, AB, CANADA

2 NHetC, Rutgers University, Piscataway, NJ 08854-0849 USA 
3 Physics Department, University of Maryland, College Park, MD, USA

${ }^{4}$ Department of Physics, University of Toronto, Toronto, On, Canada

${ }^{5}$ Department of Mathematics, Ohio State University, Columbus, OH, USA

${ }^{6}$ Kavli Institute for the Physics and Mathematics of the Universe (WPI), The University of Tokyo Institute for Advanced Study, The University of Tokyo, Kashiwa, Chiba 277-8583, Japan 